\title{
Contribution to Research on Underwater Sensor Networks Architectures by Means of Simulation
}

Thesis by

José Salvador Climent Bayarri

In Partial Fulfilment of the Requirements

for the Degree of Doctor of Philosophy in Computer Science

Thesis supervisor:

Juan Vicente Capella Hernández

December 2013 

A tots aquells que m'han ajudat i m'han donat suport i recolzament durant aquests quatre anys. A tots vosaltres, de tot cor, moltes gràcies. 



\section{Abstract}

The concept of smart environment envisions a world in which various kinds of smart devices collaborate towards a common objective. In this context, smart refers to the ability to acquire and apply knowledge autonomously to achieve this objective, while environment refers to the physical world. Therefore, a smart environment can be defined as one that acquires knowledge of its surroundings, and applying it can improve the experience of its inhabitants.

Ubiquitous or pervasive computing will allow this concept of smart environment to become a reality. Usually, ubiquitous computing refers to the use of small, inexpensive devices distributed in the physical world, which are able to communicate with each other and collaboratively solve a problem.

When this communication is performed wirelessly, these devices form a Wireless Sensor Network (WSN). These networks have increasingly attracted attention due to their wide range of applications, ranging from military solutions to home applications.

This thesis focuses on Underwater Wireless Sensor Networks (UWSN) in which, although sharing the same principles as the WSN, the different transmission medium changes their main means of communication from radio to acoustic signals. This change renders both networks different in many aspects such as propagation delay, available bandwidth, energy consumption, etc. In fact, acoustic signals have a propagation speed that is five orders of magnitude lower than radio signals. Therefore, many algorithms and protocols need to be adapted or re-designed.

Since the deployment of these types of networks can be very difficult and expensive, carefully planning the necessary hardware and algorithms is essential. Simulations are a very convenient way of testing different variables before actual deployment. However, this can only be achieved when using accurate models and real parameters that enable the extraction of trustworthy results and conclusions.

This thesis proposes a UWSN ecosystem based on freely available, open source tools. This ecosystem is composed of an energy-harvesting model and a low-cost, low-power underwater wake-up modem model that, alongside existing models, en- 
ables the performance of accurate simulations by providing real weather and marine conditions from the location where the target application is to be deployed.

This ecosystem is then successfully applied to the study and evaluation of various transmission protocols when applied on a real application for monitoring an offshore fish-farming facility, which is part of an Spanish research project (CICYT CTM2011-2961-C02-01). Subsequently, by using the energy-harvesting model, the simulation platform is used to determine the energy requirements of the application and to extract the minimum hardware requirements. 


\section{Resum}

El concepte d'entorn intel-ligent concebeix un món on diferents tipus de dispositius intel-ligents col-laboren per aconseguir un objectiu comú. En aquest concepte, intel-ligent fa referència a l'habilitat d'adquirir coneixement i aplicar-lo de forma autònoma per aconseguir l'objectiu comú, mentre que entorn fa referència al món físic que ens envolta. Per tant, un entorn intel-ligent es pot definir com aquell que adquireix coneixement del seu voltant i aplicant-lo permet millorar l'experiència dels seus habitants.

La computació ubiqua o generalitzada permetrà que aquest concepte d'entorn intel-ligent es faça realitat. Normalment el terme de computació ubiqua fa referència a l'ús de dispositius distribuïts per al món físic, xicotets i de baix preu, que poden comunicar-se entre ells i resoldre un problema de forma col-laborativa.

Quan aquesta comunicació es duu a terme sense emprar fils, aquestos dispositius formen una xarxa de sensors sense fils o en anglès, Wireless Sensor Network (WSN). Aquestes xarxes atrauen cada vegada més atenció degut a l'ampli espectre d'aplicacions que tenen, des de solucions per a l'àmbit militar fins a aplicacions per al gran consum.

Aquesta tesi es centra en les xarxes de sensors subaquàtiques i sense fils o en anglès, Underwater Wireless Sensor Networks (UWSN). Aquestes xarxes, a pesar de compartir els mateixos principis que les WSN, tenen un medi de transmissió diferent que canvia la seua forma de comunicació de ones de ràdio a ones acústiques. Aquest canvi fa que ambdues xarxes siguen diferents en molts aspectes com el retard de propagació, l'ample de banda disponible, el consum d'energia, etc. De fet, els senyals acústics tenen una velocitat de propagació cinc ordres de magnitud menor que els senyals de ràdio. Per tant, molts algorismes i protocols necessiten adaptar-se o fins i tot redissenyarse.

Com el desplegament d'aquest tipus de xarxes pot ser bastant complicat i car, es deu planificar acuradament el maquinari i els algorismes que es necessiten. Amb aquesta finalitat, les simulacions poden resultar una forma molt convenient de provar totes les variables necessàries abans del desplegament de l'aplicació. 
No obstant això, un nivell de precisió adequat que permeta extraure resultats i conclusions confiables, solament es pot aconseguir utilitzant models acurats i paràmetres reals.

Aquesta tesi proposa un ecosistema per a UWSN basat en ferramentes lliures i de codi obert. Aquest ecosistema es composa d'un model de recol-lecció d'energia i un model d'un módem de baix cost i baix consum amb un sistema d'activació remota que, junt amb altres models ja implementats en les ferramentes, permet la realització de simulacions acurades amb dades ambientals del temps i de les condicions marines del lloc on l'aplicació objectiu d'estudi s'ha de desplegar.

Seguidament, aquest ecosistema s'utilitza amb èxit en l'estudi i l'avaluació de diferents protocols de transmissió aplicats a una aplicació real de monitorització d'una piscifactoria a la costa del mar Mediterrani, que és part d'un projecte de recerca espanyol (CICYT CTM2011-2961-C02-01). Finalment, utilitzant el model de recol-lecció d'energia, aquesta plataforma de simulació s'utilitza per mesurar els requisits d'energia de l'aplicació i extraure les necessitats mínimes de maquinari. 


\section{Resumen}

El concepto de entorno inteligente concibe un mundo donde los diferentes tipos de dispositivos inteligentes colaboran para conseguir un objetivo común. En este concepto, inteligencia hace referencia a la habilidad de adquirir conocimiento y aplicarlo de forma autónoma para conseguir el objetivo común, mientras que entorno hace referencia al mundo físico que nos rodea. Por tanto, un entorno inteligente se puede definir como aquel que adquiere conocimiento de su entorno y aplicándolo permite mejorar la experiencia de sus habitantes.

La computación ubicua o generalizada permitirá que este concepto de entorno inteligente se haga realidad. Normalmente, el término de computación ubicua hace referencia al uso de dispositivos distribuidos por el mundo físico, pequeños y de bajo precio, que pueden comunicarse entre ellos y resolver un problema de forma colaborativa.

Cuando esta comunicación se lleva a cabo de forma inalámbrica, estos dispositivos forman una red de sensores inalámbrica o en inglés, Wireless Sensor Network (WSN). Estas redes están atrayendo cada vez más atención debido al amplio espectro de aplicaciones que tienen, des de soluciones para el ámbito militar hasta aplicaciones para el gran consumo.

Esta tesis se centra en las redes de sensores inalámbricas y subacuáticas o en inglés, Underwater Wireless Sensor Networks (UWSN). Estas redes, a pesar de compartir los mismos principios que las WSN, tienen un medio de transmisión diferente que cambia su forma de comunicación de ondas de radio a ondas acústicas. Este cambio hace que ambas redes sean diferentes en muchos aspectos como el retardo de propagación, el ancho de banda disponible, el consumo de energía, etc. De hecho, las señales acústicas tienen una velocidad de propagación cinco órdenes de magnitud menor que las señales de radio. Por tanto, muchos algoritmos y protocolos necesitan adaptarse o incluso rediseñarse.

Como el despliegue de este tipo de redes puede ser bastante complicado y caro, se debe planificar de forma precisa el hardware y los algoritmos que se necesitan. Con esta finalidad, las simulaciones pueden resultar una forma muy conveniente 
de probar todas las variables necesarias antes del despliegue de la aplicación. A pesar de eso, un nivel de precisión adecuado que permita extraer resultados y conclusiones confiables, solamente se puede conseguir utilizando modelos precisos y parámetros reales.

Esta tesis propone un ecosistema para UWSN basado en herramientas libres y de código abierto. Este ecosistema se compone de un modelo de recolección de energía y un modelo de un módem de bajo coste y bajo consumo con un sistema de activación remota que, junto con otros modelos ya implementados en las herramientas, permite la realización de simulaciones precisas con datos ambientales del tiempo y de las condiciones marinas del lugar donde la aplicación objeto de estudio va a desplegarse.

Seguidamente, este ecosistema se utiliza con éxito en el estudio y evaluación de diferentes protocolos de transmisión aplicados a una aplicación real de monitorización de una piscifactoría en la costa del mar Mediterráneo, que es parte de un proyecto de investigación español (CICYT CTM2011-2961-C02-01). Finalmente, utilizando el modelo de recolección de energía, esta plataforma de simulación se utiliza para medir los requisitos de energía de la aplicación y extraer las necesidades de hardware mínimas. 


\section{Contents}

Abstract $\quad$ I

Resum III

Resumen V V

Contents $\quad$ VII

List of Figures $\quad$ XI

List of Tables $\quad$ XVII

1 Introduction 1

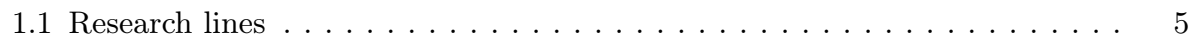

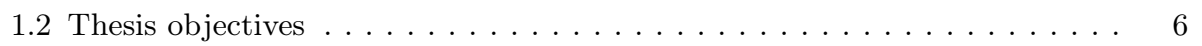

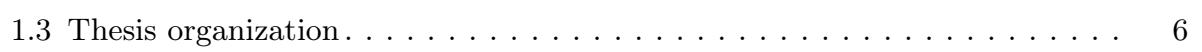

2 Related Work 9

2.1 Basics of the Underwater Physical Layer . . . . . . . . . . . . . 9

2.1 .1 Underwater Acoustic Channel . . . . . . . . . . . . . . . . . . . . 10

2.1.2 Commercial and Research Modems . . . . . . . . . . . . . . . . . 11

2.1 .3 Acoustic Triggered Wake-Up Systems . . . . . . . . . . . . . . . 11

2.2 Medium Access Control Layer. . . . . . . . . . . . . . . . . . . . . . . . . . . . 12

2.2.1 Frequency-Division Multiple Access. . . . . . . . . . . . . . . . . . . 13 
2.2.2 Code-Division Multiple Access . . . . . . . . . . . . . . . . . . . . 15

2.2 .3 Time-Based Schemes. . . . . . . . . . . . . . . . . . . . . . . . . . . 17

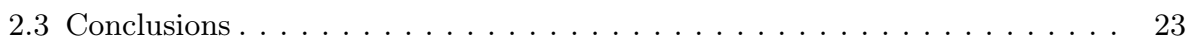

3 Underwater Simulation Platform 25

3.1 Underwater Simulation Tools . . . . . . . . . . . . . . . . . . . . 25

3.1.1 Underwater Model of the ns-3 Simulator. . . . . . . . . . . . . . . . . . . 26

3.1.2 The Bellhop Channel Model and the WOSS API . . . . . . . . . . . . 27

3.2 Low-Power, Wake-Up Modems . . . . . . . . . . . . . . . . . . . . . . 28

3.2 .1 Wills Underwater Modem. . . . . . . . . . . . . . . . . . . . . . . . . . . 28

3.2.2 ITACA-S1000 Underwater Modem . . . . . . . . . . . . . . . . . . . 28

3.3 Low-power Underwater Wake-up Modem Model . . . . . . . . . . . . . . . . 29

3.4 Wake-up Systems Evaluation . . . . . . . . . . . . . . . . . . . . . . . 31

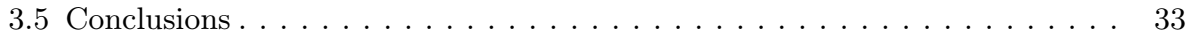

4 Energy-Harvesting Simulation Platform 35

4.1 Energy Model of the ns-3 Simulator. . . . . . . . . . . . . . . . . . 36

4.2 Energy-Harvesting Model for the ns-3 Simulator . . . . . . . . . . . . 37

4.2 .1 Capacitor energy source. . . . . . . . . . . . . . . . 37

4.2 .2 Radio energy model . . . . . . . . . . . . . . . . . . . 40

4.2 .3 Sensor energy model . . . . . . . . . . . . . . . . . . . . 40

4.2 .4 Solar panel energy model . . . . . . . . . . . . . . . . . . . . 41

4.3 Model Evaluation . . . . . . . . . . . . . . . . . . . . . 41

4.3 .1 Solar panel characterization . . . . . . . . . . . . . . 42

4.3 .2 Stressful testbench . . . . . . . . . . . . . . . . . . 43

4.3 .3 3-Day outdoor Test. . . . . . . . . . . . . . . . . . . . . . 44

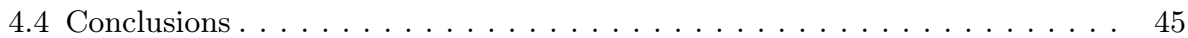

5 Experimentation 47

5.1 Case Study . . . . . . . . . . . . . . . . . . . . 47

5.2 Simulation Experiments with MAC Protocols and the Wake-Up Modem . . 48

5.2 .1 Simulation Parameters. . . . . . . . . . . . . . . . . . . . . . . 49

5.2 .2 Throughput vs backoff study. . . . . . . . . . . . . . 50

5.2 .3 Study of delay vs backoff . . . . . . . . . . . . . . . . 53

5.2 .4 Study of node energy vs backoff . . . . . . . . . . . . . . . 54 


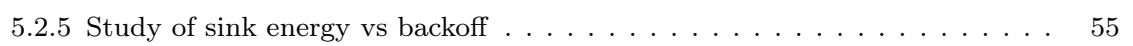

5.2 .6 Estimation of optimum backoff . . . . . . . . . . . . . . . . . 55

5.2 .7 Influence of acknowledgements on protocol performance . . . . . . . . 57

5.2 .8 Influence of transmission speed on protocol performance . . . . . . . . . 61

5.2 .9 Comparison of protocol performance . . . . . . . . . . . . 65

5.2 .10 Conclusions. . . . . . . . . . . . . . . . . . . 69

5.3 Simulation Experiments and Results Using the Energy-Harvesting Module. 70

5.3 .1 Wireless Sensor Network Scaling . . . . . . . . . . . . . . . . . . 71

5.3 .2 Simple Energy Aware Policies . . . . . . . . . . . . . . . . . . . . . 74

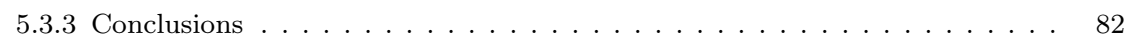

5.4 Simulation of a Real Underwater Application . . . . . . . . . . . . . . . 82

6 Conclusions 85

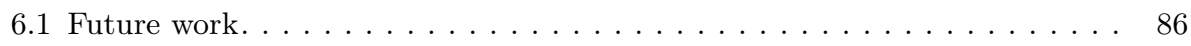

6.2 Publications Related to this Thesis . . . . . . . . . . . . . 87

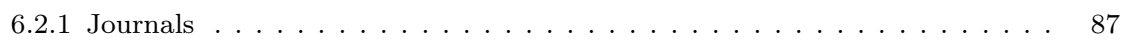

6.2 .2 International Conferences . . . . . . . . . . . . . . . . . . 89

A Simulation Results of the MAC Study 93

A.1 Thoughput vs backoff study. . . . . . . . . . . . . . . . . . . . 94

A.2 Delay vs backoff study . . . . . . . . . . . . . . . . . . . . 99

A.3 Energy vs backoff study . . . . . . . . . . . . . . . . . . . . . . . . . . . 104

A.4 Sink energy vs backoff study . . . . . . . . . . . . . . . . . . . 109

A.5 Estimation of the optimum backoff . . . . . . . . . . . . . . . . 114

A.6 Influence of acknowledgements on throughput. . . . . . . . . . . . . . . . . 119

A.7 Influence of acknowledgements on delay . . . . . . . . . . . . . . . . . 122

A.8 Influence of acknowledgements on node's energy . . . . . . . . . . . . . . 125

A.9 Influence of acknowledgements on sink's energy . . . . . . . . . . . . . . . . . 128

A.10 Influence of transmission speed on protocol throughput . . . . . . . . . 131

A.11 Influence of transmission speed on node energy consumption . . . . . . . . . 133

A.12 Influence of transmission speed on sink energy consumption . . . . . . . . 135

$\begin{array}{ll}\text { Bibliography } & 137\end{array}$ 



\section{List of Figures}

1.1 Underwater Wireless Sensor Network typical architecture . . . . . 4

2.1 Contention-free communication can be accomplished by assigning time, frequency, or unique codes. . . . . . . . . . . . . . 13

2.2 Classification of MAC protocols . . . . . . . . . . . . 15

3.1 ns-3 modules . . . . . . . . . . . . . . . . . 26

3.2 Simplified component diagram of the ns-3 underwater model . . . 27

3.3 Simplified component diagram of the underwater wake-up model . 29

3.4 Interaction diagram showing the interaction between the radio model and the wake-up model . . . . . . . . . . . . . 30

3.5 Wills Modem acoustic wave transmitter diagram . . . . . . . . . 31

3.6 Comparison of the two different modems . . . . . . . . . . . . . . 34

4.1 Simplified class diagram of the ns-3 original energy model . . . . . 37

4.2 Simplified class diagram of the ns-3 energy model . . . . . . . . . 38

4.3 Energy-harvesting module diagram block . . . . . . . . . . . 38

4.4 Solar panel current (Ipanel mean per hour) . . . . . . . . . . . . 43

4.5 Sensor node simplified state diagram. Where each state duration is defined in Table 4.3 and $\mathrm{S}$ is the node Sleep time (60 s by default).

4.6 Capacitor voltage in discharge tests: real prototype measures (blue) and ns-3 model (red) . . . . . . . . . . . . . . . . . . 44 
4.7 Solar energy conditions during the model validation experiments . $\quad 45$

4.8 Real test results and model estimation . . . . . . . . . . . . . . . 46

5.1 Diagram of the simulation scenario . . . . . . . . . . . . . 48

5.2 Aloha throughput vs backoff analysis . . . . . . . . . . . . . 50

5.3 FAMA throughput vs backoff analysis . . . . . . . . . . . . 51

5.4 MACA throughput vs backoff analysis . . . . . . . . . . . . . 53

5.5 Aloha delay vs backoff analysis . . . . . . . . . . . . . . 54

5.6 Aloha energy vs backoff analysis . . . . . . . . . . . 55

5.7 Aloha sink energy vs backoff analysis . . . . . . . . . . . . 56

5.8 Aloha optimum backoff estimation . . . . . . . . . . . 56

5.9 Comparison of Aloha with and without ACK . . . . . . . . . 58

5.10 Comparison of FAMA with and without ACK . . . . . . . . . 59

5.11 Comparison of MACA with and without ACK . . . . . . . . 60

5.12 T-Lohi with and without ACK comparison . . . . . . . . . 61

5.13 Influence of transmission speed on Aloha throughput . . . . . . . . 62

5.14 Influence of transmission speed on Aloha energy consumption . . . 62

5.15 Influence of transmission speed on FAMA throughput . . . . . . 63

5.16 Influence of transmission speed in MACA throughput . . . . . . . 64

5.17 Influence of transmission speed on T-Lohi throughput . . . . . . . 64

5.18 Influence of transmission speed on T-Lohi energy consumption . . 65

5.19 Comparison of protocol throughput without acknowledgements . . 66

5.20 Comparison of protocol throughput with acknowledgements . . . . 66

5.21 Comparison of protocol energy without acknowledgements . . . . . 67

5.22 Comparison of protocol energy with acknowledgements . . . . . . 68

5.23 Comparison of protocol energy sink without acknowledgements . . 68 
5.24 Comparison of protocol energy sink with acknowledgements . . . . 69

$5.25 \mathrm{TX} / \mathrm{RX}$ cycle variation . . . . . . . . . . . . . . . . 71

5.26 Increasing the RX time by $m=4$ and $m=5$ times without hysteresis 72

5.27 Threshold voltage depending on capacitor capacitance and energy consumption .................... 73

5.28 Increasing the RX time by $m=5$ times with and without hysteresis 74

5.29 Capacitance variation . . . . . . . . . . . . . 75

5.3024 hours, 365 days solar radiation integrated energy $\left(\mathrm{x} 10 \frac{\mathrm{kJ}}{\mathrm{m}^{2}}\right) .0$ hours corresponds to maximum solar power time. Day 0 corresponds to January 1 st . . . . . . . . . . . . . . . . . 76

5.31 Node max. and min. voltages during one year . . . . . . . . . . . . 77

5.32 Node max. and min. voltages when applying the proposed policy during one year . . . . . . . . . . . . . . . 80

5.33 Voltage level and sleep time . . . . . . . . . . . . . . . . 81

A.1 Aloha throughput vs backoff analysis . . . . . . . . . . . . . . . 94

A.2 Aloha with ACK throughput vs backoff analysis . . . . . . . . 95

A.3 FAMA throughput vs backoff analysis . . . . . . . . . . . . 95

A.4 FAMA with ACK throughput vs backoff analysis . . . . . . . 96

A.5 FAMA X2 throughput vs backoff analysis . . . . . . . . . . 96

A.6 FAMA X2 with ACK throughput vs backoff analysis . . . . . . . . 97

A.7 MACA throughput vs backoff analysis . . . . . . . . . . . . . . 97

A.8 MACA with ACK throughput vs backoff analysis . . . . . . . . . 98

A.9 Aloha delay vs backoff analysis . . . . . . . . . . . . . . . . . . . 99

A.10 Aloha with ACK delay vs backoff analysis . . . . . . . . . . . . 100

A.11 FAMA delay vs backoff analysis . . . . . . . . . . . . . . 100

A.12 FAMA with ACK delay vs backoff analysis . . . . . . . . . . 101

A.13 FAMA X2 delay vs backoff analysis . . . . . . . . . . . . . . 101 
A.14 FAMA X2 with ACK delay vs backoff analysis . . . . . . . . . . . 102

A.15 MACA delay vs backoff analysis . . . . . . . . . . . . . . . 102

A.16 MACA with ACK delay vs backoff analysis . . . . . . . . . . . 103

A.17 Aloha energy vs backoff analysis . . . . . . . . . . . . . . . 104

A.18 Aloha with ACK energy vs backoff analysis . . . . . . . . . . . 105

A.19 FAMA energy vs backoff analysis . . . . . . . . . . . . 105

A.20 FAMA with ACK energy vs backoff analysis . . . . . . . . . . . 106

A.21 FAMA X2 energy vs backoff analysis . . . . . . . . . . . . . 106

A.22 FAMA X2 with ACK energy vs backoff analysis . . . . . . . . . . 107

A.23 MACA energy vs backoff analysis . . . . . . . . . . . . . . . 107

A.24 MACA with ACK energy vs backoff analysis . . . . . . . . . . 108

A.25 Aloha sink energy vs backoff analysis . . . . . . . . . . . . . . . 109

A.26 Aloha with ACK sink energy vs backoff analysis . . . . . . . . . 110

A.27 FAMA sink energy vs backoff analysis . . . . . . . . . . . . . . . 110

A.28 FAMA with ACK sink energy vs backoff analysis . . . . . . . . . . 111

A.29 FAMA X2 sink energy vs backoff analysis . . . . . . . . . . . 111

A.30 FAMA X2 with ACK sink energy vs backoff analysis . . . . . . . . 112

A.31 MACA sink energy vs backoff analysis . . . . . . . . . . . . . . . . 112

A.32 MACA with ACK sink energy vs backoff analysis . . . . . . . . . . 113

A.33 Aloha optimum backoff analysis . . . . . . . . . . . . . . . . . . 114

A.34 Aloha with ACK optimum backoff analysis . . . . . . . . . . . 115

A.35 FAMA optimum backoff analysis . . . . . . . . . . . . . . . 115

A.36 FAMA with ACK optimum backoff analysis . . . . . . . . . . . 116

A.37 FAMA X2 optimum backoff analysis . . . . . . . . . . . . . 116

A.38 FAMA X2 with ACK optimum backoff analysis . . . . . . . . . . . 117

A.39 MACA optimum backoff analysis . . . . . . . . . . . . . . . 117 
A.40 MACA with ACK optimum backoff analysis . . . . . . . . . . . . . 118

A.41 Acknowledgements influence on throughput Aloha . . . . . . . . . 119

A.42 Acknowledgements influence on throughput FAMA . . . . . . . . 120

A.43 Acknowledgements influence on throughput MACA . . . . . . . . 120

A.44 Acknowledgements influence on throughput T-Lohi . . . . . . . . . 121

A.45 Acknowledgements influence on delay Aloha . . . . . . . . . . . . . 122

A.46 Acknowledgements influence on delay FAMA . . . . . . . . . . 123

A.47 Acknowledgements influence on delay MACA . . . . . . . . . . . . 123

A.48 Acknowledgements influence on delay T-Lohi . . . . . . . . . . . . 124

A.49 Acknowledgements influence on nodes's energy - Aloha . . . . . . . 125

A.50 Acknowledgements influence on nodes's energy - FAMA . . . . . . 126

A.51 Acknowledgements influence on nodes's energy - MACA . . . . . . 126

A.52 Acknowledgements influence on nodes's energy - T-Lohi . . . . . . 127

A.53 Acknowledgements influence on sink's energy - Aloha . . . . . . . . 128

A.54 Acknowledgements influence on sink's energy - FAMA . . . . . . . 129

A.55 Acknowledgements influence on sink's energy - MACA . . . . . . . 129

A.56 Acknowledgements influence on sink's energy - T-Lohi . . . . . . . 130

A.57 Transmission speed influence on throughput Aloha . . . . . . . . . 131

A.58 Transmission speed influence on throughput FAMA . . . . . . . . . 131

A.59 Transmission speed influence on throughput FAMA X2 . . . . . . . 132

A.60 Transmission speed influence on throughput MACA . . . . . . . 132

A.61 Transmission speed influence on throughput T-Lohi . . . . . . . . 132

A.62 Transmission speed influence on Aloha node energy consumption . 133

A.63 Transmission speed influence on FAMA node energy consumption . 133

A.64 Transmission speed influence on FAMA X2 node energy consumption 134

A.65 Transmission speed influence on MACA node energy consumption 134 
A.66 Transmission speed influence on T-Lohi node energy consumption 134

A.67 Transmission speed influence on Aloha sink energy consumption 135

A.68 Transmission speed influence on FAMA sink energy consumption . 135

A.69 Transmission speed influence on FAMA X2 sink energy consumption 136

A.70 Transmission speed influence on MACA sink energy consumption . 136

A.71 Transmission speed influence on T-Lohi sink energy consumption . 136 


\section{List of Tables}

2.1 Protocol classification and properties . . . . . . . . . . . . . 14

3.1 Energy consumption of the Wills and ITACA radios . . . . . . . 33

4.1 Radio current consumption . . . . . . . . . . . . . . 42

4.2 Sensor current consumption . . . . . . . . . . . . . . . . 42

4.3 3-day test Benchmark . . . . . . . . . . . . . . . . 44

5.1 Underwater modem energy consumption . . . . . . . . . . . . . . 49

5.2 Packet statistics with and without energy aware policy in two different locations . . . . . . . . . . . . . . . . 78

5.3 Policy variables values . . . . . . . . . . . . . . . . . . 79

5.4 Final scenario simulation results . . . . . . . . . . . 83 



\title{
Chapter 1
}

\section{Introduction}

\begin{abstract}
ПHe Internet of Things (IoT) will enable the objects around us to be inter1 connected and accessible at any time and from anywhere. The European Research Cluster on the Internet of Things (IERC) defines IoT as "A dynamic global network infrastructure with self-configuring capabilities based on standard and interoperable communication protocols where physical and virtual things have identities, physical attributes, and virtual personalities and use intelligent interfaces, and are seamlessly integrated into the information network" [1].

In this definition, the word dynamic is used to highlight the fact that these networks are changing and evolving, which implies that the interconnected objects have to include self-configuring capabilities. These objects are able to sense or even actuate with the environment, identifying themselves in order to bring meaningful data to the information network.
\end{abstract}

The IoT is not a technology, it is a concept which is enabled by many different technologies, such as semantic data and semantic search to process the enormous amounts of data collected, Machine-to-Machine (M2M) communications, sensor networks, and energy-harvesting technologies, just to name a few.

Sensor networks, and in particular Wireless Sensor Networks (WSNs), can contribute significantly to this concept of the IoT. A WSN consist of tiny sensor nodes, which act as both data generator and network relays and collaboratively sense and deliver the requested data to a sink node. Because of their wide ranging applicability, they have attracted a lot of attention. In [2], Libelium, a leading company in WSNs solutions, envisions 50 ways in which sensor networks can be utilized, for example, in smart cities, environment monitoring, smart agriculture and farming, and even in eHealth. 
Sensor networks that are utilized underwater are usually called Underwater Wireless Sensor Networks (UWSNs). Although they share the same principles as their terrestrial counterparts, the particular characteristics of the underwater environment introduce new challenges for the research community. The objective of this thesis is to contribute to this vast world of the IoT by studying, modelling, and analysing the performance of Underwater Wireless Sensor Networks.

Underwater acoustic communication has been under research for at least half a century now. One of the first underwater communication devices was the underwater phone, developed for the US Navy after World War II [3].

During the last decade, this form of communication has received increased attention motivated by many scientific, military, and commercial interests because it can enable a broad range of applications, such as the following:

- Environmental Monitoring. UWSNs can perform pollution or biological monitoring and can even help to predict weather forecasting and climate change.

- Seismic Monitoring. Monitoring seismic activities in oil extraction underwater facilities is very important.

- Disaster Prevention. By performing seismic monitoring, a UWSN can provide tsunami warnings to coastal areas.

- Assisted Navigation. Sensor networks can be used to detect the roughness of the seafloor and to assist in the navigation of AUVs. Sensors can also assist the localization applications in AUVs by functioning as beacons.

- Undersea Exploration. UWSNs can aid exploration of the sea floor by, for example, performing bathymetry profiling and assisting navigation of underwater vehicles.

- Tactical Surveillance. By collaboratively monitoring areas and assisting the navigation of underwater vehicles, UWSNs can be used for intrusion detection and surveillance.

The traditional approach to ocean monitoring consists of deploying underwater sensors that record the necessary data and, subsequently, recover, retrieve, and process them. The main disadvantage of this approach is the off-line operation, which does not allow for:

- ReAl-time monitoring. The data can only be accessed when the sensors are retrieved from the ocean.

- Failure detection. Not being able to perform real-time monitoring, it is not possible to detect failures during the operation of the sensor nodes. 
- Online ReCOnfiguration. Real-time configurations would allow the user to reconfigure the nodes when a failure occurs or when the requirements change.

- Low-COST maintenance. The need to physically retrieve the nodes for reconfiguration and data retrieval increases the maintenance cost, especially in off-shore installations.

These disadvantages can be overcome by underwater sensor communications. Electromagnetic waves, optical waves, and acoustic waves have been successfully used in underwater wireless sensor networks (UWSNs) [4] [5]. However, radio frequency $(\mathrm{RF})$ waves are affected by high attenuation in water (especially at higher frequencies) [4], thus requiring high transmission power and large antennae [6]. Optical waves can be used to achieve ultra-high-data-rate communications (Gbit/s) but are rapidly scattered and absorbed in water, so they are only reliable for shortdistance links [4].

In contrast, acoustic waves enable communications over long-range links because their susceptibility to absorption is relatively low. Thus, this is the preferred technology to develop reliable UWSNs [5] and is the central focus of this thesis. In [7], the characteristics of the acoustic underwater channel and underwater communication difficulties are discussed. The differences between acoustic and radio-based communication open a new research field in UWSNs.

Some of the challenges that still need to be overcome in order to deploy an underwater acoustic network are (i) the limited bandwidth available, (ii) the huge propagation delay, (iii) the high cost of available hardware, and (iv) the limited battery power.

Despite the increased complexity of acoustic transmissions when compared to RF transmissions, researchers have made enormous advances since the underwater telephone. Nowadays, commercially available underwater modems are able to transmit up to $30 \mathrm{kbps}$ over distances ranging from hundreds of metres to a few kilometres [8][9].

Given these advances in underwater transmission capabilities, an increasing amount of research has been focused on building networks of underwater nodes. Because of the long propagation delays that exist in this environment, direct use of the medium access control (MAC) and routing protocols of conventional RF networks is not advisable. Hence, a great deal of research has been focused on this issue. Moreover, some of these protocols require time synchronisation and localization. These problems must be revisited because propagation time is not usually taken into account in RF networks.

The typical architecture of an underwater sensor node is depicted in Figure 1.1. It usually consists of a main micro-controller unit, which is typically a System-on- 


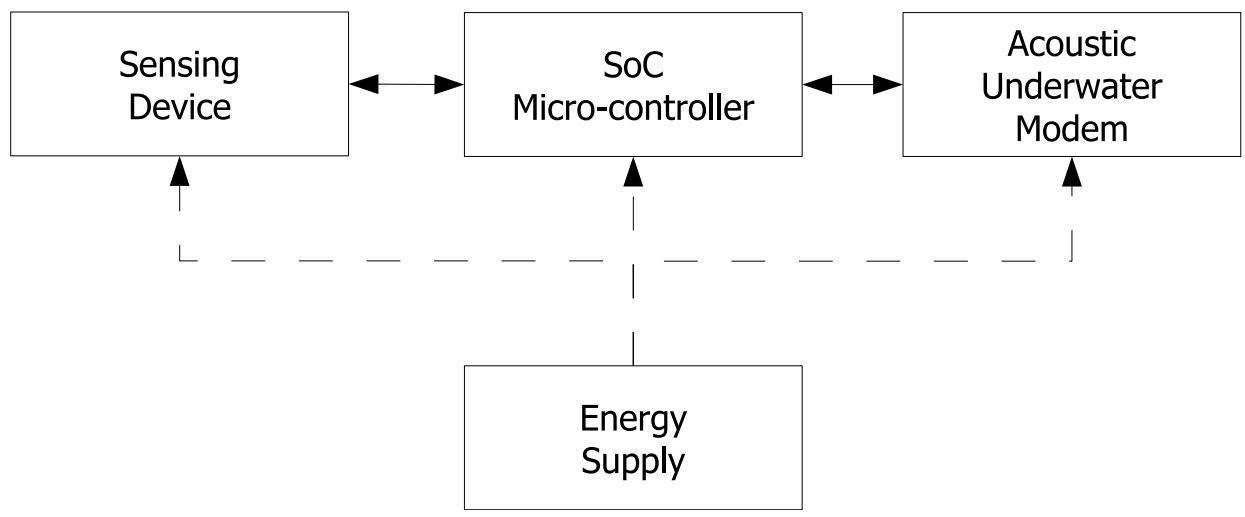

Figure 1.1: Underwater Wireless Sensor Network typical architecture

Chip (SoC) design, including RAM and flash memory and various input/output systems - SPI, UART, $\mathrm{I}^{2} \mathrm{C}$, etc. This micro-controller is connected to one or more sensing devices $\left(\mathrm{CO}_{2}\right.$, temperature, salinity, etc. $)$ and to an acoustic modem. The micro-controller receives data from the sensors, which it processes prior to sending them via the acoustic modem to another device in the network, typically a sink node or an intermediate node, which in turn, has to perform routing.

Generally, for a typical UWSN to be successfully deployed, the following are requisite:

- Self-adaptation. A UWSN must be able to react to the continuously changing environment and perform in an autonomous manner giving selfadapting decisions.

- Self-organization. The network should have self-organizing features in order to support node movements and topology changes.

- Self-COnfiguration. In order to reinforce the self-organization capabilities, the communication protocols have to be able to adapt and reconfigure themselves autonomously to support the changing topology.

- Self-optimization. These types of networks are usually very resourceconstrained; hence, optimal usage of the computational power and energy supply are mandatory.

- Self-energy-harvesting. In always-available networks, battery powered devices are expensive to maintain and sometimes it may even be impossible to replace the batteries. Hence, in some applications, nodes might need to include energy-harvesting capabilities in order to build everlasting sensor networks. 


\section{$1.1 \quad$ Research lines}

This thesis was developed in the "Redes de Sensores y Sistemas Empotrados" group in the ITACA institute at the Universitat Politècnica de València. The group is dedicated to applied and scientific research in the field of wireless sensor networks and embedded systems. It also offers consultancy services and viability studies for technological projects.

The members of the group comprise computer, industrial, and telecommunication engineers, full-time researchers, highly qualified technicians, and university students. It has conducted research activities in close cooperation with the national and European industry for more than 25 years. The group accounts for highly-equipped software and hardware laboratories, fully prepared for designing and building all types of prototypes and systems. Its main research areas are as follows:

1. Wireless AND UNDERWATER SEnsor Networks. In this research line different topics are being studied in the field of Wireless and Underwater Networks: specifically, routing algorithms, fault tolerance, energy-efficient algorithms and low-power hardware design.

2. Industrial Data Networks, Control, and Monitoring. This research line studies and implements industrial communication systems for controlling and monitoring production systems.

3. High-Reliability Embedded Systems Design. This research line develops and implements hardware and software for high reliability environments.

4. Energy-Harvesting Systems. This research line studies new and existing techniques to harvest energy for wireless and underwater networks.

5. Cache Memory applichtions in Real Time Systems. The central focus of this research line is to analyze how advances in cache microprocessors can be adapted to real time applications.

This thesis falls under research lines 1 and 4 and its main objectives are modelling and evaluating efficient low-power underwater wireless sensor networks with energy-harvesting capabilities.

The research projects related to wireless and underwater sensor networks in which the group has been involved since the start of this thesis in 2009 are the following:

- Dependable design and manufacturing for embedded systems.

- Design, evaluation, and implementation of a UWSN. 
- Basic research in WSN building technologies and their application in developing a WSN platform.

- Research on reliability improvement of WSN applications developing a hybrid monitoring platform.

- UWSN ambient monitoring for fish farming facilities.

- Research on intelligent protocols for the improvement of WSN reliability.

\subsection{Thesis objectives}

The central purpose of this research is to accurately model and develop a simulation ecosystem for low-power underwater wireless sensor networks with energyharvesting capabilities by using freely available, high quality, open source tools. The specific objectives are as follows:

- Study the state of the art of UWSN. In order to fully understand the requirements of these networks, the physical layer needs to be studied, and MAC protocols analysed and evaluated.

- Accurately model a real low-power underwater modem in order to provide reliable underwater simulation results.

- Accurately model a real energy-harvesting system in order to obtain reliable energy simulation results.

- Study the behaviour of various medium access protocols under different circumstances by using the previously implemented models.

- Study and evaluate a real application using both models and determine its energy requirements.

\subsection{Thesis organization}

After outlying the introduction, objectives and motivation of this research work, the remainder of the dissertation is organized as follows:

Chapter 2 gives a comprehensive view of the current MAC for underwater wireless sensor networks research. It gives a brief overview of the physical layer and an in-depth analysis of the MAC layer. Finally, conclusions on the current state of the art in MAC protocols for UWSN and future challenges to be faced are presented.

Chapter 3 introduces a model of an underwater modem. The model is based on previously implemented high quality physical models and a real, fully functional, 
hardware modem. Subsequently, Chapter 4 presents an easily extendable energyharvesting model based on solar power and super-capacitors and also inspired by an actual operational energy-harvesting platform.

Chapter 5 demonstrates the utility of these simulation models by evaluating the behaviour of various medium access control protocols under different circumstances when they are adapted for a low-power, wake-up modem. In addition, energyharvesting simulations are performed in various parts of the world to show the importance of accurately estimating the available energy before deployment. The final section of the chapter determines the energy requirements of a real UWSN application.

Finally, Chapter 6 outlines the conclusions, possible future research directions, and the publications related to this thesis. 



\section{Chapter 2}

\section{Related Work}

Sate-of-the-art in UWSN comprise many research areas: channel modelling $S$ and characterization [7], security in communications [10], time synchronization [11], node localization [12], collision avoidance strategies [13], routing and topology control [14], etc.

This chapter focuses on the state-of-the-art of medium access control (MAC) protocols for UWSN, although a brief introduction to the underwater physical layer is given to provide a general idea of its special features and currently available modem characteristics.

\subsection{Basics of the Underwater Physical Layer}

The physical layer defines the mechanism to transmit bits over a physical link channel connecting network nodes. The transmitter converts bit streams into a physical signal that is propagated through the physical layer. On the other side, the receiver should be able to reverse the process and provide the original bit stream to the upper communication layers.

The goal of physical layer design is to provide robust and reliable communications with the highest spectrum efficiency, which is the ratio of the communication bit rate to the channel bandwidth. Additionally, because UWSN nodes are usually autonomous and powered by batteries, power saving is also a key physical layer issue. 


\subsubsection{Underwater Acoustic Channel}

The propagation speed of an acoustic signal underwater is on average approximately $1500 \mathrm{~m} / \mathrm{s}$; however, the actual value depends on the salinity, temperature, and pressure of the medium, among other factors. A complete nine-term equation for calculating propagation speed is defined in [15], as shown in (2.1), where $T$ denotes the temperature in degrees Celsius; $D$, the depth in meters; and $S$, the salinity in parts per thousand of the water.

$$
\begin{array}{r}
c=1448.96+4.591 T-5.304 \times 10^{-2} T^{2}+2.374 \times 10^{-4} T^{3} \\
+1.340(S-35)+1.630 \times 10^{-2} D+1.675 \times 10^{-7} D^{2} \\
-1.025 \times 10^{-2} T(S-35)-7.139 \times 10^{-13} D^{3}
\end{array}
$$

The path loss of the signal can be modelled as $(2.2)$, where $f$ is the signal frequency and $l$ is the transmission distance. The path-loss component $k$ models the spreading loss and is usually between 1 and 2 . The absorption coefficient can be obtained using an empirical formula as described in [16] and is expressed in (2.3).

$$
\begin{gathered}
A(l, f)=l^{k} a(f)^{l} \\
10 \log a(f)=\frac{0.11 f^{2}}{\left(1+f^{2}\right)}+\frac{44 f^{2}}{\left(4100+f^{2}\right)}+0.000275 f^{2}+0.0003
\end{gathered}
$$

The ambient noise $(N)$ is dependent on the deployment environment. For ocean environments, empirical formulas exist which model the noise from four sources: turbulence $\left(N_{t}\right)$, shipping $\left(N_{s}\right)$, waves $\left(N_{w}\right)$, and thermal noise $\left(N_{t h}\right)$ [17]. Formula (2.4) give the power spectral density of the four noise components in $\mathrm{dB}$ re $\mu \mathrm{Pa}$ per $\mathrm{Hz}$ as a function of frequency $(f)$ in $\mathrm{kHz}$. In addition, $s$ is the shipping factor, which ranges from 0 to 1 for low and high activities, respectively, and $w$ is the wind speed in $\mathrm{m} / \mathrm{s}$ :

$$
\begin{aligned}
10 \log N_{t}(f) & =17-30 \log f \\
10 \log N_{s}(f) & =40+20(s-0.5)+26 \log (f)-60 \log (f+0.03) \\
10 \log N_{w}(f) & =50+7.5 w^{1 / 2}+20 \log f-40 \log (f+0.4) \\
10 \log N_{t h}(f) & =-15+20 \log f \\
N(f) & =N_{t}(f)+N_{s}(f)+N_{w}(f)+N_{t h}(f)
\end{aligned}
$$

As introduced in previous works [5] [18], the overall effect of the transmission loss $(A(l, f))$ and the noise density $(N(f))$ are evaluated as the narrow-band signal-tonoise ratio (SNR) for different distances and different frequencies. In conclusion, 
both frequency-dependent absorption and noise and slow propagation speed have a significant impact on the design of underwater communication networks.

\subsubsection{Commercial and Research Modems}

In contrast to RF wireless sensor networks, only a few companies are engaged in developing commercial modems for underwater sensor networks. These modems are the result of modern ongoing research, with several publications in relevant international conferences [19] [20] [21]. Moreover, these companies have collaborated with underwater researchers in recent marine projects, such as European FP5, FP6, and FP7 in the case of Aquatec [22]; ONR US projects in the case of WHOI [23]; and private contractors in the case of Teledyne Benthos [9].

These modems characteristics range from extremely low bit rates, 40 bps in case of the TriTech modem [24], to relatively high ones like the LinkQuest modem working at $35 \mathrm{kbps}$ [25]. Transmission distance has also a great variability, ranging from a few hundred of meters to several kilometres [25].

In order to improve the trade-off between transmission distance, available bandwidth and power consumption, research in underwater acoustic modems is still ongoing [26] [27] [28]. These modems are focused on low rage communications with bit rates ranging from a few hundred bps to several kbps.

\subsubsection{Acoustic Triggered Wake-Up Systems}

In normal operation, UWSN nodes alternate data communication and IDLE modes. In an ideal scenario, nodes remain switched off as long as no communication is requested, so minimal energy is consumed. However, synchronisation based on clock sharing strategies presents an additional energy waste to the minimum required to transmit or receive [29]. Moreover, in an event-driven communication, which starts when some specific event is recognised, the wireless interface tends to remain active longer than is necessary to complete a packet transmission.

The UWSN can save significant power when an acoustic-triggered wake-up (ATWU) system is used. Using this technique, a node is able to react to certain acoustic signals, reactivating the node if needed. However, it requires specific hardware to perform this task and it is crucial that this new hardware dissipates ultra-low power, as it always remains active.

Very little work has been done on asynchronous AT-WU, though there are various state-of-the-art commercial modems that implement this technology. Main references so far to the research of acoustic modems enhanced with AT-WU are the Wills modem [30] and ITACA modem [31]. The power consumptions of these 
modems when waiting for an acoustic wake-up signal are $500 \mu W$ and $10 \mu W$, respectively.

\subsection{Medium Access Control Layer}

MAC protocols manage access to the communication medium. Without proper management of the transmission medium, collisions of unrequested communications may degrade overall network performance. Hence, the basic objective of MAC protocols is to avoid collisions. However, they also have to deal with other factors, such as energy efficiency, scalability, and latency.

A collision occurs when two or more data frames arrive at the intended receiver simultaneously. Traditional MAC protocols try to handle this time uncertainty by, for example, slotting time (time-division multiple access, or TDMA) or sensing the channel prior to transmission (carrier sense multiple access, or CSMA). However, because of the long propagation delays of underwater transmission, these networks also suffer from space uncertainty, and it is necessary to take into account the locations of the receivers and their possible interferers. This problem is commonly known in the literature as space-time or spatio-temporal uncertainty [32].

Another problem that comes along with long propagation delays is spatial unfairness [32]. Because the packet reception time depends on the distance to the transmitter, the channel becomes free first at the transmitter and later on at the receiver. Hence, nodes closer to the transmitter are able to gain access to the channel before nodes closer to the receiver.

Nevertheless, despite these problems, the two classical schemes in which MAC protocols can be subdivided, i.e. contention-free and contention-based schemes, are still valid and used.

Contention-free schemes assign different frequency bands, time slots, or codes to different users of the communication medium. Under this scheme, nodes do not compete with each other in order to obtain access to the channel. The three basic types of this scheme, i.e. TDMA, frequency-division multiple access (FDMA), and code-division multiple access (CDMA), are illustrated in Figure 2.1.

Contention-based MAC protocols, on the other hand, avoid the pre-allocation of resources by allowing nodes to compete with each other and obtain medium access on demand. This group of protocols usually relies on random access to distribute transmissions and normally also includes some recovery mechanisms in case a collision occurs.

This classification, although widely accepted, is not entirely accurate because there exist different protocols that share characteristics of both schemes. In Figure 2.2, 


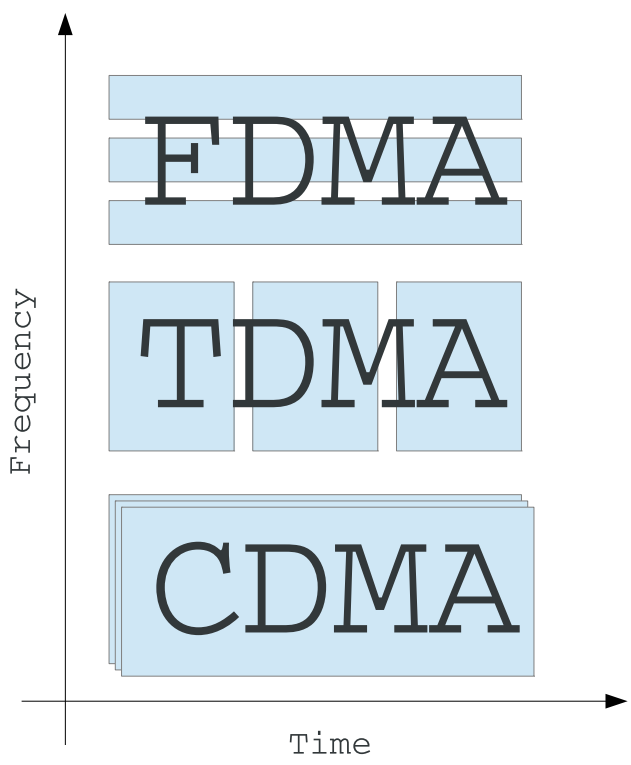

Figure 2.1: Contention-free communication can be accomplished by assigning time, frequency, or unique codes.

a representative classification is shown. The presented protocols are further explained in the remainder of this section, and their main properties are compared in Table 2.1. This table specifies the type of MAC protocol (time-based, FDMAbased, or CDMA-based), whether the protocol uses a random access scheme (CDMA scheme, TDMA scheme, or a combination), if the nodes are organised in clusters, whether the MAC protocol also performs routing, if the MAC protocol requires a handshaking process, and finally, if time synchronisation is needed or knowledge of the propagation time is required.

\subsubsection{Frequency-Division Multiple Access}

FDMA is a contention-free medium access scheme that divides the available bandwidth into different frequency bands, allowing different nodes to transmit and receive at the same time while avoiding collisions. However, these frequency bands have to be carefully assigned and used because collisions may occur inside the same band as well. For example, if each node is assigned a receiving frequency, when two or more nodes try to reach the same destination at the same time, a collision will occur.

This scheme was used in the early phases of the Seaweb project [33]. Three clusters were deployed and within each cluster, TDMA was used, and FDMA was chosen 


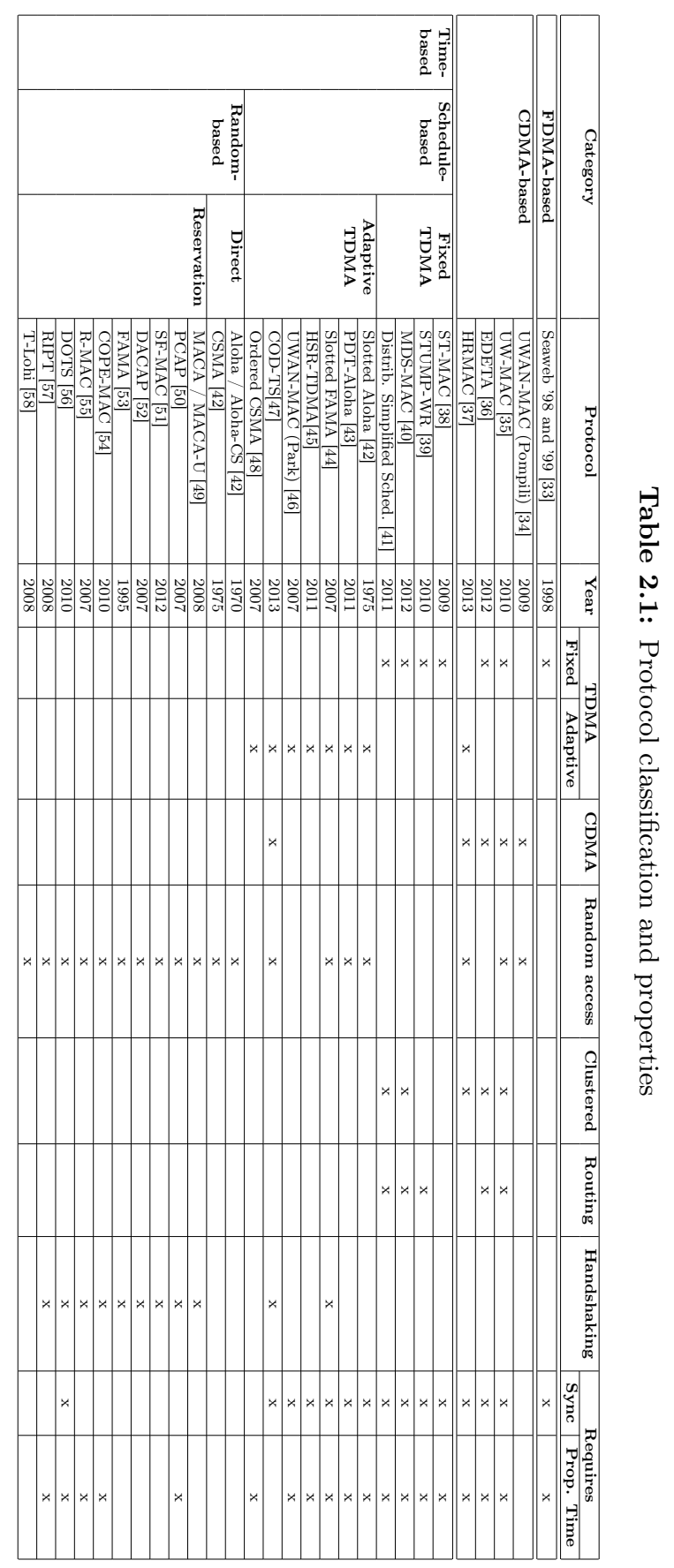




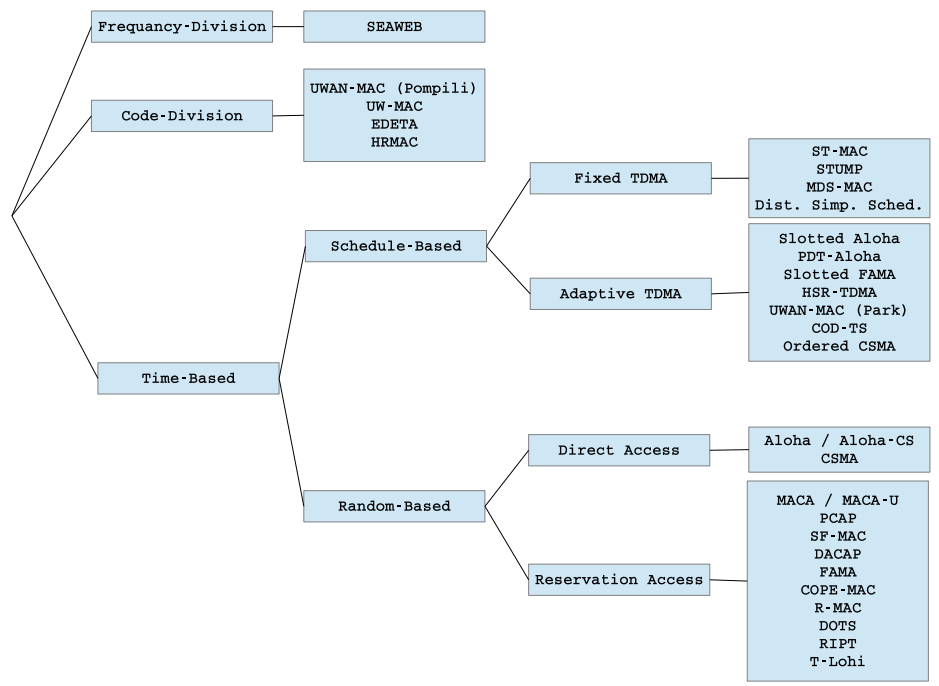

Figure 2.2: Classification of MAC protocols

for inter-cluster communications. However, an inefficient use of bandwidth was reported, and the protocol was also vulnerable to fading and multipath. Since then, FDMA has been considered to be unsuitable for underwater acoustic networks [59] [6].

\subsubsection{Code-Division Multiple Access}

The basic principle of the CDMA scheme is the use of binary codes to modulate the signal using a spread-spectrum technique. When different nodes transmit using different codes with low cross-correlation, their data can be received simultaneously in the same frequency band by the other nodes in the network without collision. The advantage of this scheme over FDMA is that it does not suffer from selective fading because it uses the entire frequency band. Its advantage over TDMA is that the transmission medium can be accessed at the same time by all users. However, low cross-correlation implies long codes, which greatly reduce the available data rate $[60]$.

It is shown in [60] how the available data rate per user $\left(V_{t x}\right)$ depends on the code length $(L)$ and the actual modem data rate $\left(V_{t x}^{\prime}\right)$ :

$$
V_{t x}=\frac{V_{t x}^{\prime}}{L}
$$


It is also shown how code length $(L)$ depends only on the number of different codes $(N)$ as shown in expression $(2.6)$ :

$$
L=4^{\log _{2} N}-1
$$

Using expressions (2.5) and (2.6), the effective transmission speed $\left(V_{t x}\right)$ of different users of a network can be calculated. For example, given a network of only eight nodes equipped with an acoustic modem capable of transmitting at $1000 \mathrm{bps}$, the effective transmission speed of each node would roughly be 16 bps.

Nonetheless, different authors use the properties of CDMA to their advantage. Pompili et al. proposed a combination of CDMA and aloha in [34]. A node willing to transmit dynamically calculates the transmission power and spreading code length in order to efficiently communicate with its intended receiver. This spreading code is then sent using aloha without any sort of coding. Immediately after that, the data packet is sent using the spreading code.

CDMA codes can be used for intra-cluster communication in a clustered network. In [35], the authors propose a cluster-based network in which each cluster has its own CDMA spreading code assigned. Communication inside each cluster is executed by exchanging request-to-send (RTS) and clear-to-send (CTS) packets, and cluster heads communicate with the sink node using TDMA.

Another cluster-based approach is presented in [36] in which, similar to the previous work, each cluster uses its own CDMA spreading code for intra-cluster communication. Transmissions inside the cluster are scheduled using TDMA. Instead of directly communicating to the sink node, cluster heads arrange themselves in a tree structure in order to send their collected data to the sink.

Following this cluster-based proposal, the hybrid reservation-based MAC protocol (HRMAC) [37] utilises an adaptive TDMA along with CDMA spreading codes. Each node with data to transmit sends a notice packet with sender and destination IDs and data size to the cluster head. With this information, the cluster head computes a sending schedule and populates it. Upon receiving the transmission schedule, nodes send their data packets in their assigned slots. Afterwards, the receivers of the data packets send a reply message back to the sender with the amount of data received. The CDMA codes are used for the notice and reply packets because they are not scheduled and can collide. 


\subsubsection{Time-Based Schemes}

Protocols based on this scheme use the complete bandwidth for a certain amount of time, so multiple transmissions have to be distributed in time by scheduling them, reserving the channel time prior to transmission, or directly sending the data packet. Specifically, there are mainly two different strategies to avoid collisions:

1. Scheduled-based: a time interval or frame is divided between all nodes. With this strategy, two techniques are normally used:

(a) Fixed TDMA: each node is assigned a time period where it is able to transmit.

(b) Adaptive TDMA: time periods are assigned on demand either by dynamically assigning the slots by some coordinator or by allowing the nodes to contend for the slots.

2. Random-based: selections of the transmission start and end times are arbitrary, and nodes directly compete for channel acquisition. This group of protocols can also be sub-divided into

(a) Direct. Protocols under this group send data directly without performing any channel reservation.

(b) Reservation. Prior to the transmission of the actual data packet, nodes reserve the channel using control packets.

\section{Scheduled-Based Schemes}

In the schedule-based protocols, each node is assigned a time period in which it is able to transmit. This technique requires synchronisation between all nodes, which can be done using a synchronisation algorithm [11]. In addition, in order to guarantee a contention-free communication, it might be necessary to include guard times. The duration of these guard times depends on the maximum propagation delay and the synchronisation accuracy, which degrade the network performance.

There are basically two types of schedule-based protocols, fixed TDMA and adaptive TDMA. In fixed TDMA, each of these time periods is assigned to a node, and the node is only able to transmit during this time.

Given the large propagation delays in the underwater medium, it is possible that packets from two different nodes arrive successfully, even if the packets were transmitted at the same time [32]. Based on this, different approaches have been proposed that try to schedule the TDMA-based transmissions in such a way that they can overlap without conflicting at the intended receivers. 
In [38], the authors propose spatial-temporal MAC (ST-MAC), which formulates the TDMA-based scheduling problem as a vertex-colouring problem. The algorithm constructs a spatial-temporal conflict graph describing the conflict delays among transmission links. Afterwards, an optimal solution is proposed based on a mixed integer linear programming model, and a new heuristic approach is proposed to solve the vertex-colouring problem.

The staggered TDMA underwater MAC protocol (STUMP) is a similar approach introduced in [61]. A set of TDMA scheduling constraints is derived, and the authors propose centralised and distributed algorithms in order to solve the scheduling problem. They first determine the order of transmissions between the conflicting nodes. Once the order is fixed, the scheduling constraints become a system of difference equations, which is solved using the Bellman-Ford algorithm. In a subsequent work [39], the authors further improve their proposal by adding routing capabilities.

A recent alternative is multi-dimensional scaling MAC (MDS-MAC), proposed in [40]. It integrates time-synchronisation, localisation, and communication scheduling for small underwater clusters. The operation of the protocol is divided into coordination and communication phases, which are repeated periodically. During the coordination phase, nodes perform range measurements in order to calculate the propagation delay between them and to achieve relative localisation and time synchronisation. At the beginning of the communication phase, the cluster head broadcasts the communication schedule and routing information. Afterwards, during the remainder of this communication period, all nodes within the network follow this schedule.

A clustering scheduling approach is described in [41]. Spatial-temporal communication scheduling is performed within clusters by the cluster head. The cluster heads forward the length of the complete schedule to a central scheduler, which assigns time to the different clusters. By allowing the cluster heads to schedule within the cluster, the central scheduler does not need to know the positions of all nodes within the network to guarantee a collision-free schedule. This reduces the otherwise very significant overhead of spatial-temporal communication scheduling.

Ordered CSMA [48] schedules transmissions through ordering. Every node in the transmission chain waits until it has detected the carrier of the preceding node in the schedule. After detecting the carrier, the node is allowed to transmit its data. By ordering the transmissions in such a way, collision-free transmissions are guaranteed.

On the other hand, adaptive TDMA protocols allow nodes to adaptively assign time periods on demand. This assignment can be done through contention and handshaking processes or by learning the transmission schedules of the neighbouring nodes. 
In slotted aloha, as in pure aloha, nodes contend for the channel [42]. However, in slotted aloha, the transmission is deferred to the beginning of each time slot. Hence, each node is obliged to schedule the beginning of its transmissions at the beginning of each time slot. Nevertheless, because slots are not assigned as in pure TDMA, collisions may also occur if different nodes select the same slot to transmit. However, given the space uncertainty of the underwater acoustic medium, the performance of this protocol is degraded to that of pure aloha. In [43], the authors try to cope with this problem by adding extra guard time in the time slots, achieving $17-100 \%$ better throughput results than the original slotted aloha in an underwater medium.

The original floor acquisition multiple access (FAMA), which is introduced in Section 2.2.3, requires long RTS and CTS packets in order to guarantee that the data packets will be transmitted collision-free [53]. However, in the underwater acoustic channel, where transmissions are expensive, excessively large control packets might be too energy expensive. In order to reduce these high energy costs, slotted FAMA uses time slots, in the same way as slotted aloha, to reduce the control packet size [44]. The slot length is equal to the maximum propagation delay plus the transmission time of a CTS packet, which assures that only control packets may collide and that the transmission of data packets is collision-free.

Another approach is the one proposed in [45], in which TDMA is used, but nodes are able to adaptively identify who can transmit at the same time without causing collision. In order to do so, nodes calculate a list of neighbours and share it by piggybacking it to their outgoing packets. Upon reception of a new list of neighbours, each node updates its connectivity matrix, and based on this, the node then decides whether or not it is able to safely transmit in the next slot.

A different proposal for low-duty-cycle underwater communication networks is given in [46]. This protocol sets up an adaptive TDMA schedule. Nodes first exchange SYNC packets within their transmission periods and learn their neighbours. Consequently, a node knows when it should wake up to hear a transmission and when there are no transmissions so that it can remain in sleep mode.

Cluster-based on-demand time sharing (COD-TS) [47] proposes a different solution. Nodes are organised into clusters, and the cluster heads are the nodes in charge of assigning the slots for the next communication round. At the beginning of each round, the cluster head populates the schedule, and each node sends its request to transmit at the end of the communication round. In addition, cluster heads communicate among themselves in order to avoid collisions with neighbouring clusters. 


\section{Random-Based Schemes}

This set of protocols avoids the pre-allocation of resources by allowing nodes to compete with each other and obtain medium access on demand. These protocols usually rely on random access to distribute transmissions. They also normally include some recovery mechanisms in case a collision occurs. Protocols under this category can be subdivided into direct access and reservation access.

Direct Access Protocols under this category do not perform any kind of handshake for channel reservation. However, they can perform carrier sensing prior to transmission in order to avoid disrupting ongoing transmissions, deferring it until the channel is free.

Aloha [42] is the simplest method to access the medium. It simply sends a packet whenever there is data to send, without performing any type of channel assessment or retransmission. In its variant called aloha with carrier sensing (aloha-CS), prior to transmission, each node performs a clear channel assessment (CCA) in order to avoid disrupting any ongoing transmission. If the channel is sensed to be free, the sending node transmits its packet. However, if an ongoing transmission is sensed, the node waits until the channel is free and then sends the packet.

Different studies have been conducted in order to understand the performance of aloha in an underwater medium. In [62], the authors develop an analytic model to study the performance of contention-based protocols by modelling different versions of aloha in an array network. Although the authors make several simplifications, different conclusions are drawn. Arrays longer than five hops are probably not going to perform well when using the simple aloha protocol. However, using p-persistent aloha without dropping packets increases the network throughput at the cost of increasing the delay.

Another study based on the aloha protocol is presented in [63], in which two different variants of aloha are proposed. Aloha with collision avoidance (alohaCA) tries to avoid collisions by overhearing the transmitted packets and knowing the propagation delays between all node pairs. The other proposed modification is aloha with advanced notification (aloha-AN), which consists of sending a short data packet prior to the actual data transmission with information on the sender and the intended receiver.

CSMA [42] is another well-known protocol under this category. Similar to alohaCS, this protocol uses carrier sensing. However, unlike aloha-CS, after waiting for the channel to be free, a node does not immediately send its packet. Instead, it performs random back-offs to mitigate the probability of collisions. This variant is called non-persistent CSMA. In another variant called p-persistent CSMA, a node transmits with a probability of $\mathrm{p}$ when the channel is sensed to be free. Note that 1-persistent CSMA is equivalent to aloha-CS. 
Reservation Access This technique consists of reserving the channel prior to transmission of the actual data packet. In order to do so, usually, short control packets are sent before transmission. By reserving the channel, the frequency of collisions of data packets is minimised, and the additional traffic introduced by the control packets is compensated for.

In the handshake-based alternative, whenever a transmitter wants to send a data packet, it first sends a control packet informing the other nodes that it has data to send. Upon reception of this control packet by the intended receiver, it replies if the channel is not being used. After receiving this reply packet, the transmitter can start the transmission of the data packet.

However, this reservation mechanism cannot guarantee collision freeness because of the well-known hidden and exposed node problems. Many authors have proposed different solutions to cope with this problem.

Multiple access collision avoidance (MACA) [64] is the first approach of a handshake algorithm proposed to reserve the channel. The sender first sends an RTS control packet in order to start the channel reservation. This packet contains the length of the data packet so that other nodes know how long they should wait until they can initiate their own transmissions. Afterwards, the receiver replies with a CTS packet. When the transmitter receives the CTS, it starts the transmission of the actual data packet. However, this algorithm does not entirely solve the exposed terminal problem, especially in channels with long propagation delays, such as the underwater channel. MACA for underwater (MACA-U) [49] adapts MACA to the long propagation delays of the underwater medium by modifying some of the state transition rules of the original protocol.

Another protocol to solve the handshaking problems in the underwater medium is the propagation-delay-tolerant collision avoidance protocol (PCAP) [50]. This protocol splits the transmission of a CTS packet so that it arrives at the transmitter after twice the maximum propagation delay. While waiting for the CTS packet, the transmitter and its neighbours can perform different actions, such as transmitting data packets or starting the handshaking process for another transmission.

Spatially fair MAC (SF-MAC) [51] also tries to avoid collisions by deferring the CTS packet transmission for a predefined amount of time. During this time, the receiver analyses all RTS packets that are sent to it and determines, based on an estimate, which node was the first to send the RTS packet and the node to which the CTS packet should be addressed.

Another random access with reservation protocol is the distance-aware collision avoidance protocol (DACAP) proposed in [52]. This protocol tries to avoid data and RTS packet collisions by deferring the data transmission for $t$ seconds after sending the RTS. This waiting time has to be chosen based on a trade-off between 
throughput and collision probability. Moreover, it also introduces a short warning packet sent by the receiver if it overhears an RTS after sending a CTS. Another approach is given by the original FAMA protocol [53], which completely prevents data packet collisions provided that the RTS and CTS frames are sufficiently long. The length of an RTS packet should be greater than the maximum channel propagation delay, and the length of the CTS packet has to be greater than the length of an RTS plus one maximum round-trip time. In order to introduce some energy savings, Molins et al. propose in [44] the slotted FAMA protocol described in Section 2.2.3.

By reserving different transmissions in one multiple reservation packet broadcasted to all neighbours and trying to arrange data transmission with several nodes, contention-based parallel reservation MAC (COPE-MAC) [54] improves channel utilisation. Moreover, neighbouring nodes can, by overhearing, learn about future scheduled transmissions and adapt their own channel utilisation to avoid collisions.

The reservation-based MAC (R-MAC) protocol is proposed in [55] and is designed for long-term monitoring applications. Nodes alternate between sleep and listen modes periodically and randomly select their schedule. The protocol requires all nodes to know the propagation delay to all of their neighbours and their listen and sleep periods. Afterwards, the protocol reserves the channel in an RTS/CTS fashion but gives higher priority to the CTS packets.

Another protocol that exploits spatio-temporal uncertainty is delay-aware opportunistic transmission scheduling (DOTS) [56], which exploits temporal and spatial reuse by learning the propagation delay to neighbouring nodes and their scheduled transmissions. In order to achieve this, nodes must be synchronised and continually overhear the channel. The protocol is based on a MACA-like random access protocol with RTS and CTS packets. By promiscuously overhearing, a node using DOTS can locally calculate the transmission and reception schedules of its neighbours and schedule on its own to avoid collisions.

Receiver-initiated packet train (RIPT) [57] is different from the previous protocols, as it employs a receiver-initiated four-way handshake mechanism. Instead of the sender, the node that initiates the handshaking process is the receiver, which informs its neighbours that it is available to receive. After that, the neighbouring nodes inform the receiver about the size of their transmissions, and with that information and the previously known propagation delay, the receiver can calculate and broadcast a transmission order. Finally, senders follow this transmission order, and the data arrives at the receiver in a sequence of packets.

Tone-lohi (T-lohi) [58] implements this technique. It automatically adapts the contention time to the number of contending nodes. The nodes send a short packet called tone prior to the actual data packet to count the number of terminals contending for the channel. If a node does not receive any other tones, it starts 
the transmission. If it receives more tones, it adapts its back-off time depending on the number of tones received.

\subsection{Conclusions}

There are a handful of MAC protocols proposed for the UWSN. However, each protocol tries to solve different issues or gives more importance to one characteristic over another. There are many different applications with many different parameters and requirements, and choosing one MAC protocol over another can be a difficult task. Further research should include guidelines to choose between the different MAC alternatives depending on the target application constraints.

In addition, MAC protocols that require synchronisation or localisation do not usually take into account the energy consumption of these two services. MDSMAC [40] is a recently proposed protocol that integrates these two services into its specifications and takes into account the extra energy consumption. Newly proposed protocols that make use of these services should integrate them into their behaviour for a complete evaluation.

Finally, future challenges in the development of MAC protocols for acoustic UWSNS shall include the use of new modem features such as wake-up systems, which can greatly reduce power consumption. Only one protocol, T-lohi [58], makes use of this new characteristic. The applicability of the wake-up system to the existing MAC protocols needs to be evaluated, and new protocols utilising this technique shall be proposed. 



\section{Chapter 3}

\section{Underwater Simulation Platform}

Towadays, acoustic modem design allows for the implementation of a low1 power reception state in which nodes continue listening on the channel but consuming very little power (much lower than the regular reception state) while those elements of the circuitry that are not currently needed remain in the sleep state [28], [30]. In this manner, the modem is able to listen and recognize particular stimuli sent prior to the actual data packet and wake up the main circuitry to receive it. In [65], the authors presented the results of a study which show that this solution is viability as regards energy-efficiency.

In this chapter, a low-power underwater wake-up model for the ns-3 simulator [66] is introduced. Using this model, two different underwater modems with integrated wake-up capabilities are compared in terms of energy consumption.

\subsection{Underwater Simulation Tools}

Several simulators that model the underwater environment and enable the simulation and study of underwater sensor networks currently exist. Aqua-Sim [67] is a simulation package for the ns-2 simulator. This package is configured in conjunction with the CMU wireless package for the ns-2 and has various MAC and routing protocols designed for UWSN that are ready for use.

Ns-miracle [68] is another simulation package for the ns-2 that enables the simulation of underwater sensor networks. This package also includes several protocols designed for UWSN and, when used in conjunction with the WOSS project, can accurately model channel characteristics by including bathymetry data, salinity, and sound speed profile. 


\begin{tabular}{|c|c|c|}
\hline protocol & application & devices \\
\hline \multicolumn{2}{|c|}{ network } \\
\hline \multicolumn{2}{|c|}{ core } \\
\hline
\end{tabular}

Figure 3.1: ns-3 modules

In this thesis, the ns- 3 simulator is used. The ns-3 is a network simulator written from scratch that enables the easy extension and development of new models for underwater simulation. It is designed to overcome the main drawbacks of the ns-2 simulator - such as the use of split objects $(\mathrm{OTcl}$ and $\mathrm{C}++)$ which do not scale well and complicate the debugging process. In addition, ns-3 includes flexible tracing and logging systems, libraries to facilitate memory management, etc.

Among its other modules, the ns-3 simulator also includes an underwater framework that accurately models channel characteristics using, in the same way as the ns-miracle, the bellhop propagation model and environmental data such as bathymetry or sound speed profiles. Like the above-mentioned simulators, the ns-3 includes a model of an underwater modem.

\subsubsection{Underwater Model of the ns-3 Simulator}

The ns-3 is a discrete-event driven simulator for computer networks [66]. The basis of its design is the component-based development paradigm, which enforces modularity and code reuse. Consequently, it offers an object aggregation system. Objects are aggregated with other objects at execution time and communicate via callbacks.

The simulator is organized in modules, as shown in Figure 3.1. The core module implements, among other things, various utilities for programmers, such as smart pointers ${ }^{1}$, support for tracing and logging, or different implementations of random variables.

The network module includes all the basic classes on top of which the various kinds of networks are built. It includes, for example, a basic Packet and Header classes, from which every packet and packet header implementation have to inherit, and a Node class, which represents a network node and is actually a container for various modules such as network interfaces, energy modules, etc.

These two modules, core and network, comprise a generic simulation core that is used by different types of networks, amongst them, sensor networks. On top of

\footnotetext{
${ }^{1}$ Smart pointers are boost-like shared objects. These pointers simply perform a reference count, and when the count is zero the memory is freed.
} 


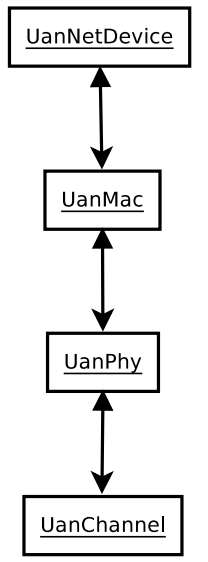

Figure 3.2: Simplified component diagram of the ns-3 underwater model

these modules, various protocols, applications, and device modules are implemented.

Among these device modules, the Underwater Acoustic Network (UAN) module is the one that implements an underwater communication device. A simplified component diagram of the ns-3 underwater modem is depicted in Figure 3.2. Following the diagram from top to bottom, the UanNetDevice component models a Network Interface Card (NIC) and is used by the upper layers to send and receive packets to/from the network. The UanMac component, which models the medium access protocol used by the nodes in the configured network, is next. This is followed by the UanPhy component, which models the underwater physical layer and includes different propagation, Signal-to-Noise-Interference Ratio (SNIR) and Packet Error Rate (PER) models. Finally, the UanChannel module models an underwater channel and delivers all the packets to the UanPhy components connected or listening to this specific underwater channel.

\subsubsection{The Bellhop Channel Model and the WOSS API}

The Bellhop channel model is a beam tracing model for predicting acoustic pressure fields underwater [69] [70]. Ray tracing requires solving of ray equations to determine the ray coordinates. It is designed to perform acoustic ray tracing for a given sound speed profile (SSP), bathymetry, and floor sediment [69].

The ns-3 simulator includes, as an add-on, an implementation of the Bellhop model. The SSP, bathymetry, and floor sediment data can be imported into the model by using the WOSS API [71] and publicly available databases like the Gen- 
eral Bathymetric Chart of the Oceans (GEBCO) [72] and National Oceanic and Atmospheric Administration (NOAA) [73].

\subsection{Low-Power, Wake-Up Modems}

To the best of our knowledge, currently there are two research modems with an integrated wake-up system: the Wills underwater modem [30] and the ITACAS1000 modem [28].

\subsubsection{Wills Underwater Modem}

The Wills underwater modem is a low-power acoustic modem designed for small range networks [30]. It includes a dedicated wake-up tone receiver and a Mica mote for packet processing and coding. When activated, this mote consumes $24 \mathrm{~mW}$ of power [74]. Its communication distance ranges from $50 \mathrm{~m}$ up to $500 \mathrm{~m}$ using frequency-shift keying (FSK) modulation at $1 k b p s$. The wake-up signal is sent in the same frequency band as the regular signal. This modem consumes at most $2 \mathrm{~W}$ when transmitting, $44 \mathrm{~mW}$ in the reception state, and $500 \mu \mathrm{W}$ in the sleep state, including the power consumed by the wake-up circuitry.

\subsubsection{ITACA-S1000 Underwater Modem}

The ITACA-S1000 modem is designed with an energy-efficient architecture for small/medium range networks, with low-power UWSN consumption constraints [28]. Its architecture is based on a microcontroler (MCU) that only consumes $24 \mathrm{~mW}$ in the reception state and $3 \mu \mathrm{W}$ in the sleep state. It is capable of transmitting up to $100 \mathrm{~m}$ using FSK modulation at $1 \mathrm{kbps}$ and consuming $120 \mathrm{~mW}$.

The acoustic wake-up signal is transmitted using on-off keying (OOK), which is compatible without additional hardware, with the FSK modulation used for the regular transmissions. Hence, this modem also transmits the wake-up signal in the same frequency band as the regular signal.

In order to be able to handle acoustic wake-up signals, the ITACA modem includes an off-the-shelf commercial peripheral, the AS3933 from Austria Microsystems [75]. Since this circuit is intended to be triggered using magnetic coupled signals, a net was specifically designed to adapt the acoustic incoming signals to the RFID based wake-up circuit. This IC with the adaptation net consumes $8.1 \mu \mathrm{W}$.

Aside from the wake-up tone capability, this modem also includes the possibility to program and send different wake-up patterns, with the same energy consumption, facilitating selective wake-up and only activating the receiving node. 


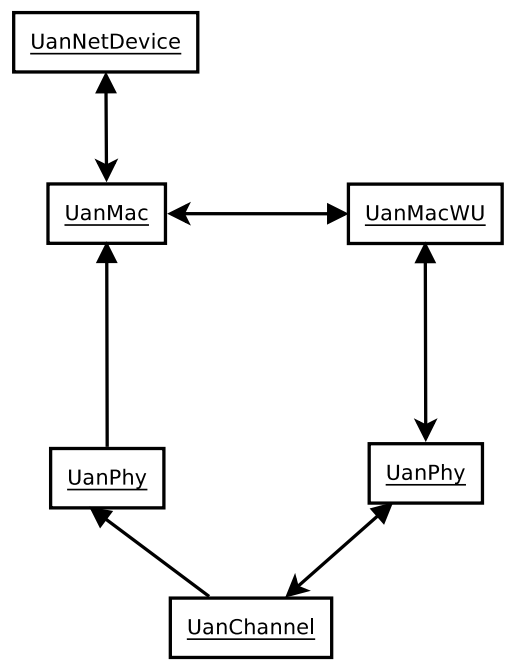

Figure 3.3: Simplified component diagram of the underwater wake-up model

\subsection{Low-power Underwater Wake-up Modem Model}

An underwater modem with wake-up capabilities enables the modem to be in an ultra-low power state and still be able to recognise certain stimuli sent prior to the current data packet and then wake the main circuitry for reception.

The presented wake-up model is based on the ITACA-S1000 and Wills modems. Figure 3.3 depicts a simplified component diagram of the wake-up modem model. As in the regular model, there is one UanNetDevice acting as an NIC card and one UanChannel to emulate the underwater channel. Two UanPhys and two UanMacs are introduced to model the regular modem and the wake-up system.

The two UanPhy modules are equivalent in terms of functionality, with the only differences being the consumption parameters, which are set to match those of the regular modem and the wake-up system.

The UanMacWU module is responsible for actual channel assessment and transmission of the wake-up packet before the packet that the UanMac module intends to send.

The UanMac module is an abstract module. Any class implementing this module should implement the medium access algorithm. The module must encapsulate the data to be sent (namely, the data from the upper layers, or the control packets such as RTS or CTS) into a UanPacket and must also ask the UanMacWU module if the channel is free. If it is free, the UanMac module sends the packet to the 
UanMacWU module. If it is not free, the UanMac module must decide what to do with this packet: discard it, back-off, etc.

When a packet is received from another node, it is always preceded by a UanPacketWU. Thus, on reception, the UanMacWU checks if the packet's destination is correct. If it is not correct, the packet is discarded. If it is correct, the UanMacWU wakes the UanPhy running the regular radio so that it can receive the packet. Figure 3.4 is a diagram showing the interaction between the regular radio model and the wake-up model.

The interaction diagram for the sending sequence is depicted in Figure 3.4a. The diagram illustrates how the UanMac module asks the UanMacWU for the current channel status and how, if the channel is free, it sends its UanPacket with the data from the upper layers, or its own control packets, to the UanMacWU. It also shows how the UanMacWU then sends the wake-up packet, immediately followed by the UanMac packet.

The receiving sequence is shown in Figure 3.4b. The UanPhyWU receives the wake-up signal from the sending node. If the signal is for this node (that is, it is a wake-up tone or a wake-up pattern that matches its own pattern), it wakes the UanPhy associated with the UanMac module so that it can receive the packet and hand it directly to the UanMac.

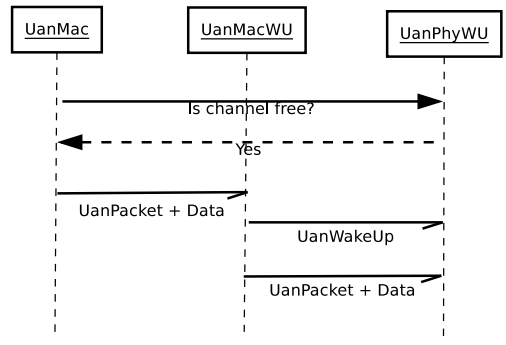

(a) Sending sequence

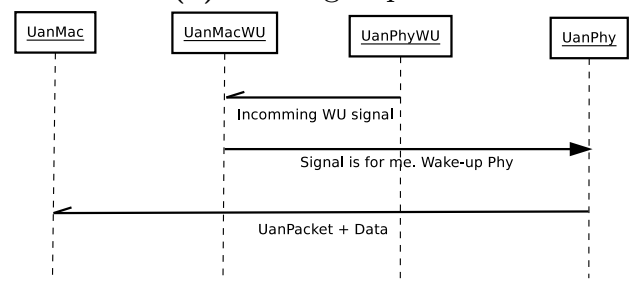

(b) Receiving sequence

Figure 3.4: Interaction diagram showing the interaction between the radio model and the wake-up model 


\subsection{Wake-up Systems Evaluation}

In this section, an evaluation of the two available research wake-up modems, i.e., Wills and ITACA-S1000, is performed using the implemented wake-up model for the ns-3 simulator.

To that end, the first step is to calculate the transmission state power consumption of the Wills modem. In the seminal paper [30] on the modem, only the energy consumption when transmitting at full-power was specified by the authors. Since the ITACA-S1000 modem is able to transmit up to distances of $100 \mathrm{~m}$, in order to perform a fair comparison, an estimation of the Wills modem energy consumption when transmitting to $100 \mathrm{~m}$ has to be performed.

From the data reported in [30], when the output power is set to $2 W$, transmission can reach $500 \mathrm{~m}$. Nevertheless, this is the maximum output level and the output power amplifier can be configured using four different gain values. The shorter the communication distance, the less power is needed to maintain the same communication performance. Therefore, to make a faithful comparison between the ITACA modem and the Wills modem, the output power has to be reduced to match 100 $\mathrm{m}$.

The transmitter of the Wills modem is shown in Figure 3.5. To calculate the amount of transmission power the Wills modem needs to reach $100 \mathrm{~m}$ communications, the following statements are assumed:

1. The Wills modem supports four discrete transmission power levels: $15 \mathrm{dBm}$, $21 \mathrm{dBm}, 27 \mathrm{dBm}$, and $33 \mathrm{dBm}[30]$.

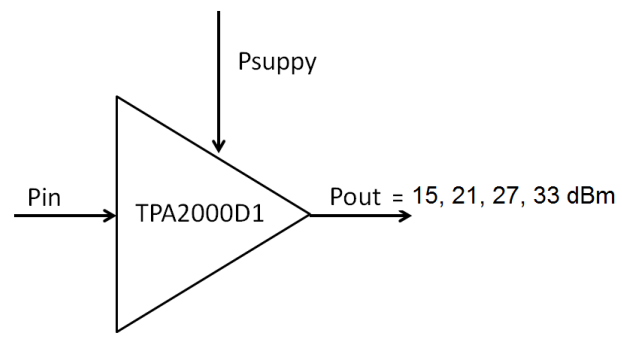

Figure 3.5: Wills Modem acoustic wave transmitter diagram 
2. Output power should be close to $108 \mathrm{~mW}(20.3 \mathrm{dBm})$, which is the power reported in [28] as being required to reach $100 \mathrm{~m}$ using the ITACA-S1000 modem.

3. Transmission power consumption is mainly due to two central blocks: power amplifier and node micro-controller. The rest of the modem elements are considered to be switched off or their power consumption is considered negligible.

The minimum value found to reach $100 \mathrm{~m}$ was reported in [28] to be $20.3 \mathrm{dBm}$. Hence, we have to choose the higher value closer to this one in the Wills power amplifier, which is $21 \mathrm{dBm}$.

The energy demanded of the power supply by the amplifier output power is obtained using the expression (3.1). The Wills output amplifier efficiency $(\eta)$ is around 0.85 [76]. Thus, it can be estimated that the Wills power consumption to transmit messages to nodes placed at a distance of $100 \mathrm{~m}$ is $148 \mathrm{~mW}(21.7 \mathrm{dBm})$. This value only accounts for the power amplifier; a Mica for which power consumption in the active mode corresponds to $24 \mathrm{~mW}$ [74], is also embedded in the architecture of the Wills modem. Hence, for $100 \mathrm{~m}$ links in TX mode, the power can be estimated at $172 \mathrm{~mW}(22.3 \mathrm{dBm})$.

$$
P_{\text {supply }}(m W)=\frac{P_{\text {out }}(m W)}{\eta_{\text {amplifier }}}
$$

The other energy consumption parameters used from the Wills modem and those from the ITACA-S1000 modem are listed in Table 3.1. The parameters are from [30] for the Wills modem and [28] for the ITACA-S1000 modem. Although these modems integrate wake-up systems, this table differentiates between the energy consumption due to the wake-up circuitry and the power consumption due to the regular modem. The reception mode consumption of the Wills wake-up system was set to zero, since this consumption is integrated in the $500 \mu \mathrm{W}$ of the sleep state of the regular modem [30]. There is no sleep mode for the wake-up, since this system is supposed to always remain awake. Nevertheless, in order to provide maximum flexibility, the wake-up model allows researchers to put the wake-up module into a sleep state and specify its energy consumption.

In order to compare the two wake-up systems, a set of simulations were performed using the proposed wake-up model with the consumption parameters specified in Table 3.1. The tests where run using the Aloha-CS medium access protocol and a simulation time of $1000 \mathrm{~s}$. Ten nodes were deployed in a $20 \times 20 \mathrm{~m}$ square area. The transmission speed was set to $1 k b p s$ and traffic generated according to a Poisson process with a generation interval varying between 1 and $100 \mathrm{~s}$. Once a packet was generated, source and destination were selected randomly using a uniform 
Table 3.1: Energy consumption of the Wills and ITACA radios

\begin{tabular}{ccccc}
\hline \hline MODE & Wills Wake-up & Wills modem & ITACA wake-up & ITACA modem \\
\hline TX mode & $172 m W$ & $172 m W$ & $120 m W$ & $120 m W$ \\
RX mode & 0 & $44 m W$ & $8.1 \mu W$ & $24 m W$ \\
IDLE mode & 0 & $44 m W$ & $8.1 \mu W$ & $24 m W$ \\
SLEEP mode & - & $500 \mu W$ & - & $3 \mu W$ \\
\hline \hline
\end{tabular}

distribution. The payload was set to $20 B$; hence, the total length of the data packet was $23 B$ and the length of the wake-up packet $1 B$.

Figure 3.6 shows the results of the simulations. Note how the ITACA-S1000 modem using the wake-up mode tone outperforms the Wills modem by saving $54 \%$ more energy with the lowest traffic rate (Wills $1.83 J$, ITACA $0.83 J$ ) and $41 \%$ with the highest traffic (Wills 55.08J, ITACA 32.40J). However, the highest performance gain comes with the wake-up pattern mode. Using this mode, only the intended receiver of the transmission wakes-up. This provides huge energy savings ranging from $74 \%$ up to $80 \%$.

Comparing this pattern mode to the optimum wake-up (where there is no energy consumption from the wake-up circuitry or from transmitting the wake-up signal), Figure 3.6 shows how the ITACA-S1000 modem with the pattern mode behaves. The results show how the wake-up energy consumption of the ITACA-S1000 modem represents only an $8 \%$ increase in the worst case.

One may want to compare these consumption values with the ones where there is no wake-up system. As a reference, the energy consumption of the ITACA-S1000 modem when there is 100 seconds of traffic generation interval and there is no wake-up system available would be approximately $24 J$, which is much higher than the $0.83 J$ achieved with the wake-up tone.

\subsection{Conclusions}

Simulations have been proven to greatly facilitate the research and development of new algorithms for underwater sensor networks. Using simulation tools, researchers can develop and test new algorithms before actual deployment, giving insights on the performance an algorithm might have in a real environment. Needless to say, the system to be simulated has to be as accurately modelled as possible to match the features of the real system.

In this chapter, a model of an underwater wake-up modem was presented. The model is designed to match the most important features of the only two research 


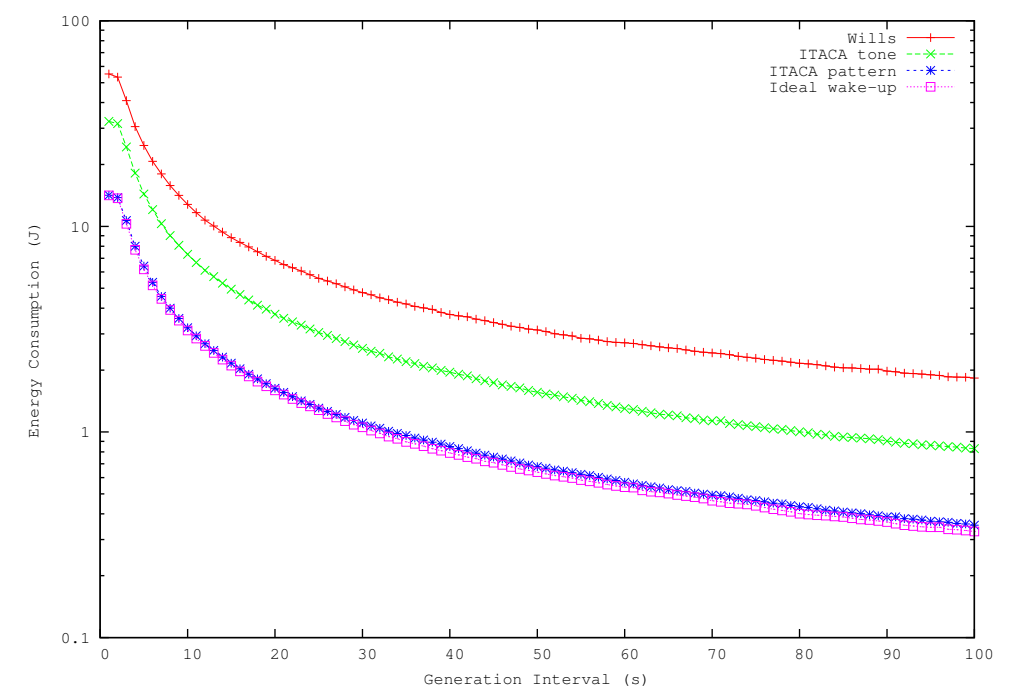

Figure 3.6: Comparison of the two different modems

wake-up systems available today, but maintain certain flexibility to be able to adapt it to new features the researchers might want to test.

Using this model, these two wake-up systems were tested and compared in terms of energy consumption to the optimum wake-up (the one in which the wake-up circuitry consumes nothing), with results showing that the ITACA-S1000 modem can provide up to $80 \%$ energy savings compared to the Wills modem and only expends $8 \%$ more energy than the optimum case.

These huge savings in energy consumption show the necessity of including these new hardware capabilities in the current protocols for underwater communication. In Chapter 5, an evaluation of existing medium access protocols modified to take advantage of this new hardware is performed. 


\section{Chapter 4}

\section{Energy-Harvesting Simulation Platform}

It is currently viable to prolong the lifetime of a sensor node indefinitely using energy-harvesting techniques combined with low-power architectures and energyaware protocols. Energy-harvesting techniques are used to collect energy from ambient sources and produce electrical energy that is stored in a buffer. These techniques are growing in importance due to their falling prices and improved technical performance - as well as accumulated scientific experience [77] [78].

Current state of the art in energy-harvesting WSNs is focused on modelling and designing energy-harvesting circuits [79] [80]. However, there has been little work done on the integration of these models into a WSN simulator. Since network simulators are not well-prepared for simulating nodes with energy-harvesting capabilities, the various networking protocols that have been proposed usually perform some type of simplification - such as assuming a uniformly distributed random variable [81] or even constant replenishment [82] [83]. In [84] the authors model the energy flow at different rates or according to different on-off processes. However, it can be difficult to describe the energy variations over long periods of time at different world locations.

Various frameworks are used to carry out power-profiling estimation of hardware [85]. However, it can be difficult to model a whole network with hundreds of nodes and numerous communication protocols (collisions, backoff time, etc).

PAWiS (power aware wireless sensors) [86] is a simulation framework developed on top of the $\mathrm{OMNeT}++$ network simulator. Only basic, but energy-aware, simulator models are available. WSNSim [87] is another energy-aware network simulator built from scratch. WSNSim includes various energy-related models such as: a 
solar panel energy model, a battery model, and a super-capacitor model. However, accuracy has not been demonstrated.

Another simulation tool has been proposed by De Mil et al. and developed over the Castalia network simulator [88]. It provides solar panel and battery models and validates them with prototype nodes. However, the prototype nodes use software to emulate the solar panel and super-capacitor.

Finally, another framework is presented in [89]. However, modelling protocols other than TDMA (time division multiple access) can be complex since the model needs to know how much time a node will be in the various radio states.

In this chapter, it is presented an energy-harvesting model based on solar power and super-capacitors for the ns-3 network simulator. The model can be easily extended to include new mechanical [90] [91] or thermal [92] [93] energy sources, as well as different storage modules such as batteries (although the ns-3 already includes a Li-ion battery model). Moreover, since the implementation is completely uncoupled from the radio model, it can be easily included with the already available models for network communication - and this allows to simulate network behaviour by only modifying the simulation scripts.

The result is a dynamic energy model since the energy available in the buffer is continuously recalculated to reflect solar radiation and the current node operating mode. The energy model uses an event-driven schedule to update radio mode changes notified by the radio model.

Moreover, it is possible to simulate several days, weeks, months, or even years, using real solar radiation curves observed in specific world locations. These data can be easily obtained from meteorological databases like AEMet [94] or PANGAEA [95].

\subsection{Energy Model of the ns-3 Simulator}

Amongst the device modules previously introduced in Chapter 3, the ns-3 includes an extensible energy model. This model is comprised of two basic classes as shown in Figure 4.1, the EnergySource class and the DeviceEnergyModel class.

The EnergySource class is an abstract class which has to be extended to model an energy source. Examples of energy sources modelled in the simulator are a Lithium ion battery, LiIonEnergySource, or an analytical non-linear battery, RvBatteryModel. The DeviceEnergyModel class is an other abstract class which has to be extended to model the energy consumption of a specific device attached to the node. There are models implemented for radio, WifiRadioEnergyModel, and acoustic communication, AcousticModemEnergyModel. 


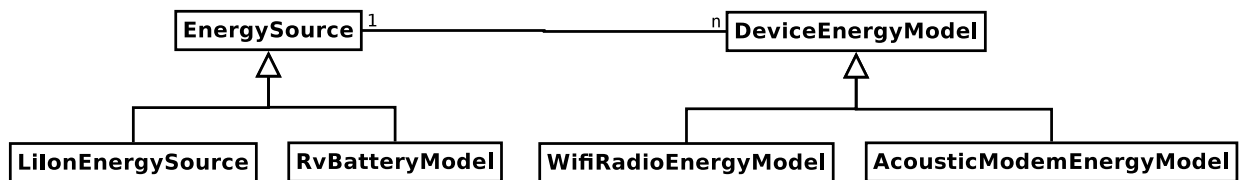

Figure 4.1: Simplified class diagram of the ns-3 original energy model

This models usually implement the different communication states (that is, transmitting, receiving, idle, sleep) and assign to each different state an energy consumption. In turn, the communication model, for example the UAN acoustic modem model, sends each state transition to the DeviceEnergyModel attached to it, following the example it would be the AcousticModemEnergyModel. When the DeviceEnergyModel receives an state transition it calculates the amount of energy used and informs the energy source in order to subtract it.

\subsection{Energy-Harvesting Model for the ns-3 Simulator}

This section introduces the implementation of an energy-harvesting model for the ns-3 simulator. Further details on how the different formulae have been derived can be obtained in [96]. Figure 4.2 depicts a simplified class diagram of the involved classes and Figure 4.3 shows the corresponding block diagram. The block diagram illustrates how the solar panel block is connected to the CapacitorEnergySource module, and this in turn, is connected to a RadioEnergyModel and a SensorEnergyModel.

The SolarPanelEnergyModel class provides energy (when it is able to harvest it) to the CapacitorEnergySource. This module calculates and manages the available energy on the actual capacitor by taking into account its voltage, the current provided by the solar panel, and the current consumed by the different elements of the node. In this case, radio and sensor energy models where developed; however, other models can be developed and easily integrated.

\subsubsection{Capacitor energy source}

Previous energy-based approaches calculate available capacitor energy by adding and subtracting harvested and consumed energy respectively [87] [97] [98]. However, as [99] demonstrates, this energy depends on instant voltage and current conditions. Hence this model accurately calculates the remaining super-capacitor energy using current and voltage values. Expression (4.1) calculates the super- 


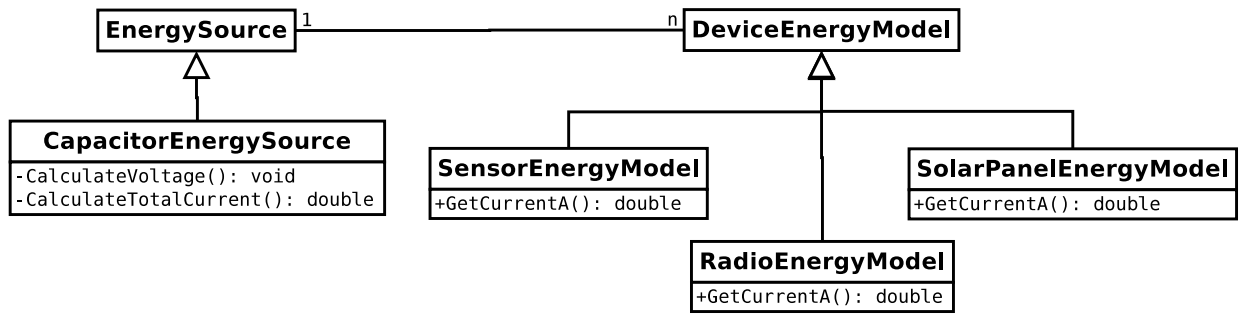

Figure 4.2: Simplified class diagram of the ns-3 energy model

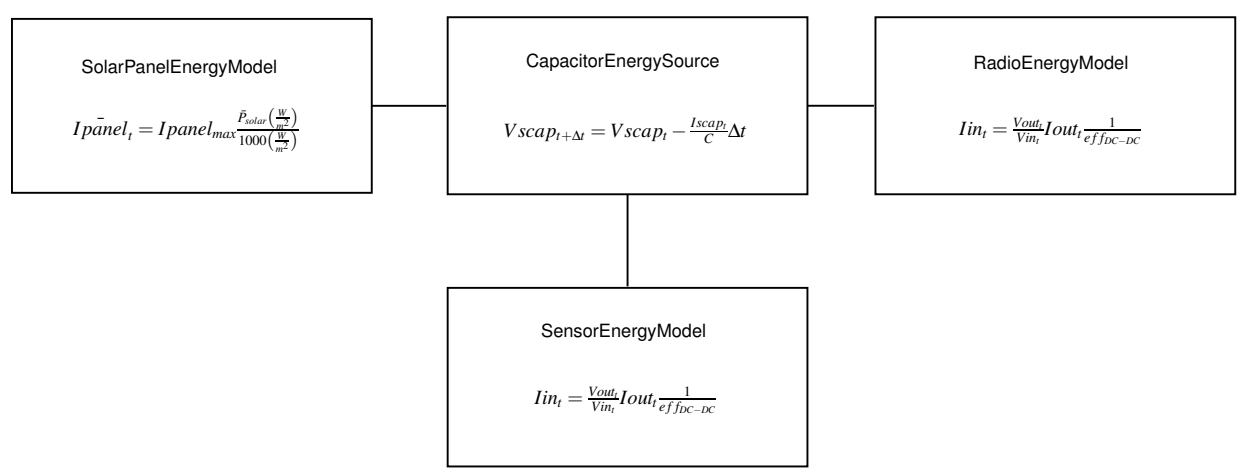

Figure 4.3: Energy-harvesting module diagram block

capacitor voltage variations, where $C$ is the actual capacitor capacity, $V$ scap the capacitor voltage at time $t$, and $\Delta t$ is the minimum time increment.

$$
V \operatorname{scap}_{t+\Delta t}=V \operatorname{scap}_{t}-\int_{t}^{t+\Delta t} \frac{I s c a p_{t}}{C} \partial t
$$

Iscap is the net current that arrives at the super-capacitor. It depends on different circuit parameters, but it can be simply calculated as the difference between the current demanded by the super-capacitor (Idis) and the current coming from the energy source (Ipanel), (4.2).

$$
\text { Iscap }_{t}=\text { Idis }_{t}-\text { Ipanel }_{t}
$$


Assuming that time intervals $(\delta t)$ are short enough to guarantee nearly constant current (Iscap) during the interval, equation (4.3) is used to describe the supercapacitor voltage level instead of the differential equation (4.1).

$$
V \operatorname{scap}_{t+\Delta t}=V \operatorname{scap}_{t}-\frac{\operatorname{Iscap}_{t}}{C} \Delta t
$$

Expression (4.3) is calculated iteratively to progressively obtain the super-capacitor voltage from the previous voltage and current consumption. Under this assumption, equation (4.3) can be seen as an approximation of equation (4.1) using the midpoint integration method. The error of applying the midpoint numerical integration method is bounded by expression (4.4).

$$
E_{V \text { scap }} \leq \frac{\Delta t^{3}}{24} \frac{\text { Iscap }^{\prime \prime}(t)}{C}
$$

It can be seen that the shorter the time interval taken for each interval, and the larger the capacitance chosen, the greater the accuracy obtained. Moreover, error decreases if the super-capacitor current is constant.

Taking a look at the actual model implementation (Figure 4.2), the CalculateVoltage method returns the voltage, calculated using expression (4.3), that a capacitor can deliver after $\Delta t$. This $\Delta t$ is a trade-off between simulation accuracy and efficiency and must be configured. In addition, the capacitor voltage is always updated whenever a change in the radio and sensor model occurs (or any other connected model).

Component demands and current delivered by the solar panels are calculated using the CalculateTotalCurrent method, which, at the same time, calls to the GetCurrent $A$ method for the current that is being used (or delivered in the case of the solar model) by each component. Both demanded and delivered currents are added using expressions (4.2) to update capacitor voltage.

As stated above, the Calculate Voltage method is always called when a mode change occurs at either radio or sensor models. Any change causes an event to recalculate capacitor energy based on the previous state (mode) of the radio (or sensor) and the amount of time since the previous calculation was performed. However, if no event is triggered during a period $\Delta t$, an automatic call to CalculateVoltage is made in order to maintain simulation accuracy. 


\subsubsection{Radio energy model}

This class models the energy consumption of a radio transceiver. Basically, it calculates the actual current consumption of the radio transceiver and communicates with the super-capacitor model.

To uncouple the implementation of the radio transceiver from the implementation of the energy model, the radio transceiver makes callbacks about its state transitions to the energy model. Hence, this radio energy model can be reused with different models of a radio transceiver by simply adjusting the consumption parameters and available states.

The current implementation includes four different states (RX, TX, IDLE and SLEEP) and a configurable amount of current can be set for each state. Moreover, since the capacitor voltage varies over time depending on its actual charge, and a node needs a fairly constant voltage to correctly operate, a DC-DC converter is included and its efficiency is taken into account when calculating the final current consumption.

Expression (4.5) is used to calculate the actual current consumption of the radio transceiver by taking into account the current consumed by the radio transceiver and the efficiency of the DC-DC converter.

$$
\operatorname{Iin}_{t}=\frac{\text { Vout }_{t}}{\text { Vin }_{t}} \text { Iout }_{t} \frac{1}{\text { eff } f_{D C-D C}}
$$

where Vout corresponds to the node operational voltage, Vin to the current capacitor voltage, Iout to the current consumed by the radio transceiver (which depends on the operational mode) and ef $f_{D C-D C}$ corresponds to the efficiency of the DC-DC converter.

\subsubsection{Sensor energy model}

This class models the energy consumption of a sensor attached to the node. This model is fundamentally the same as the radio energy model; but with only two operational modes: enabled and disabled.

In the enabled state, the sensor consumes a configurable amount of current; while consuming zero in the disabled state. Final current consumption is estimated taking into account the consumption of the DC-DC converter by using expression (4.5).

Hence, expression (4.5) is reused to calculate current sensor consumption by setting Iout to the current consumed by the sensor. 


\subsubsection{Solar panel energy model}

This class models the current delivered by solar panels. This model receives as a parameter a file containing the integrated global radiation values $\left(\frac{J}{m^{2}}\right)$ per each simulated hour.

Hourly-integrated global radiation values can be easily obtained from solar radiation databases and directly imported into the simulation tool. This procedure obtains more realistic scenarios for location conditions and meteorological variability during the day. Clouds or even eclipses can be simulated from real data. Besides, these databases are continuously updated and historical curves are publicly available.

For example, AEMet weather service database [94] stores hourly-integrated global radiation $\left(\frac{J}{m^{2}}\right)$ for several Spanish locations. Average power can be calculated using expression (4.6), which is a simple ratio between hourly-integrated energy and average power.

$$
\bar{P}_{\text {solar }}\left(\frac{W}{m^{2}}\right)=\frac{\bar{E}_{\text {solar }(\text { hourly })\left(\frac{J}{m^{2}}\right)}}{3600(s)}
$$

Another example is the PANGAEA database [95], which stores hourly-averaged direct and indirect radiation power. Assuming no reflections, hourly-averaged global radiation is the sum of both values.

Finally, actual current delivered by the solar panel is calculated in Expression (4.7). $1000\left(\frac{W}{m^{2}}\right)$ corresponds to the solar power density received on the Earth's surface with no clouds at midday and the sun in its zenith $( \pm 1.5 \%$ error $)$. The model assumes expression (4.7) using the maximum Ipanel given by the manufacturer [96].

$$
\text { Ipanel }_{t}=\text { Ipanel }_{\max } \frac{\bar{P}_{\text {solar }}\left(\frac{W}{m^{2}}\right)}{1000\left(\frac{W}{m^{2}}\right)}
$$

\subsection{Model Evaluation}

Evaluation has been carried out by comparing simulation outcomes with experimental results obtained using a prototyped hardware test bed. To avoid distortions from uncontrollable factors, tests were conducted over deterministic circumstances.

The prototyped hardware nodes have a micro-controller based design. They consist of a Texas Instruments CC1110 System-On-Chip with its radio transceiver working on the $868 \mathrm{MHz}$ frequency band [76]. Consumption parameters are shown 
Table 4.1: Radio current consumption

\begin{tabular}{cc}
\hline MODE & $\mathrm{I}$ \\
\hline TX mode & $30 \mathrm{~mA}$ \\
RX mode & $27 \mathrm{~mA}$ \\
IDLE & $27 \mathrm{~mA}$ \\
SLEEP mode & $12 \mu \mathrm{A}$
\end{tabular}

Table 4.2: Sensor current consumption

\begin{tabular}{cc}
\hline MODE & $\mathrm{I}$ \\
\hline Sensor enabled & $6 \mathrm{~mA}$ \\
Sensor disabled & $0 \mathrm{~mA}$ \\
\hline
\end{tabular}

in Table 4.1. Nodes also include a high-precision, low-power ambient temperature sensor catalogued as TI TMP $112[76]$ with $6 \mathrm{~mA}$ consumption as shown in Table 4.2 .

Hardware nodes also include a 50-farad capacitor with a maximum voltage of 2.3 $\mathrm{V}$. Node operation can start only if the capacitor voltage is above $1 \mathrm{~V}$ and the DCDC converter efficiency is measured at $92 \%$. In addition, the solar panel included in the prototype delivers $40 \mathrm{~mA}$ maximum current.

\subsubsection{Solar panel characterization}

The proposed solar panel model has been verified. Figure 4.4 shows the curves registered by the real prototype and the ns-3 simulator based on AEmet data.

The experiment was carried out on May 12, 2011. The prototype was located at the ITACA Institute in Valencia (GPS: 39.478654,-0.333595). Solar radiation data was obtained from the Manises (Valencia) Airport station (GPS: 39.492736,$0.475042)$.

Although a high correlation is shown, some inaccuracies are observed due to the $10 \mathrm{Km}$ distance between the meteorological station and the ITACA institute. As a consequence, some effects may be shifted in time. 


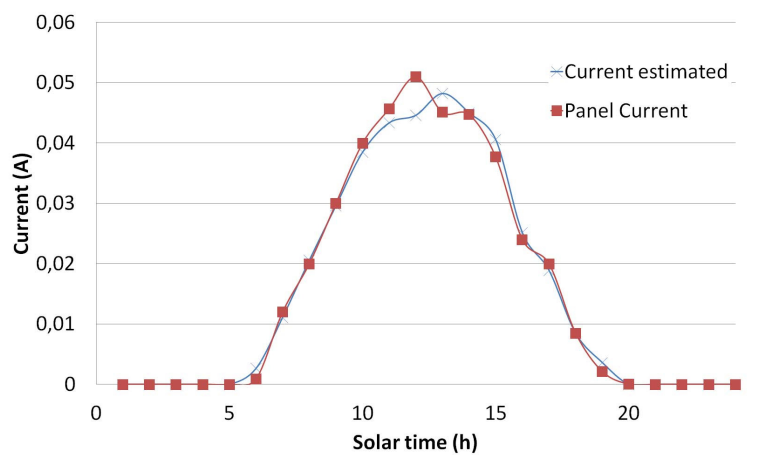

Figure 4.4: Solar panel current (Ipanel mean per hour)

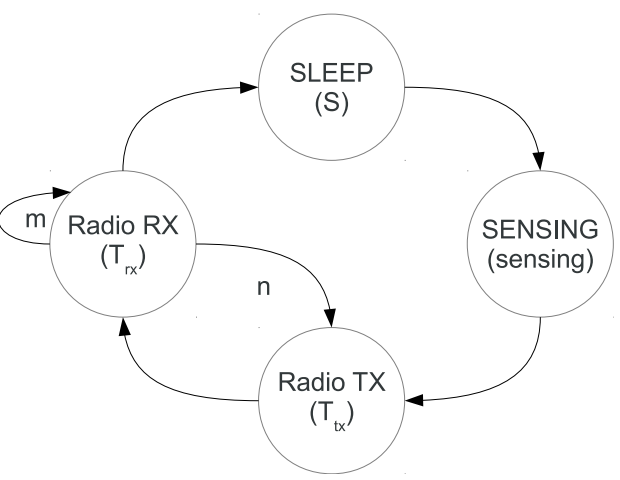

Figure 4.5: Sensor node simplified state diagram. Where each state duration is defined in Table 4.3 and $\mathrm{S}$ is the node Sleep time (60 s by default).

\subsubsection{Stressful testbench}

Several experiments have been performed discharging the capacitor under different radio energetic demands to verify the model outcomes with the real hardware prototype. The duty cycle is described in Figure 4.5. Where $\mathrm{n}$ is the number of $\mathrm{TX} / \mathrm{RX}$ cycles performed by the radio before returning to SLEEP mode again; and $\mathrm{m}$ is the number of RX cycles. The sensor is only enabled during sEnsing STATE and during this state the radio remains in sleep mode.

Three different duty cycles have been tested with 1,2 , and 5 sleep time minutes. Receive mode time was established at $500 \mathrm{~ms}$, while a transmission needs $570 \mu \mathrm{s}$. The $\mathrm{n}$ and $\mathrm{m}$ parameters were set to 0 and the remaining corresponding values are shown in Table 4.3 . 


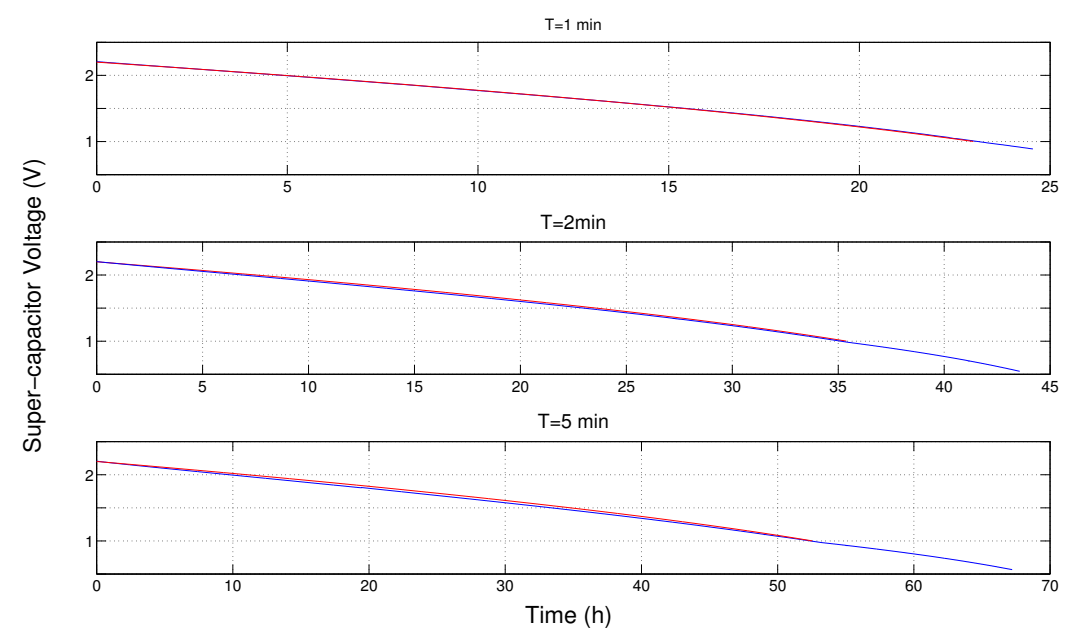

Figure 4.6: Capacitor voltage in discharge tests: real prototype measures (blue) and ns-3 model (red)

Capacitor energy was continuously powering the system up to 23,35 , and 51 hours and corresponding with 1,2 , and 5 minutes sleep time respectively. In all the experiments, the capacitor starts with maximum charge.

Figure 4.6 shows super-capacitor voltage evolution. It can be seen that the proposed analytical model fits the measurements as the maximum error is $2.3 \%$.

\subsubsection{3-Day outdoor Test}

To combine charge and discharge cycles in a real scenario, a three-day benchmark test was carried out. Experiments were run from 2 pm April 1, to 10 am April 4, 2011. Solar energy conditions are shown in Figure 4.7.

The node iterates the different states specified in Figure 4.5 cyclically - with their duration given in Table 4.3. Energy consumption is specified in Tables 4.1 and 4.2.

Table 4.3: 3-day test Benchmark

\begin{tabular}{lll}
\hline Task & Symbol & Time \\
\hline Sensing & sensing & $44 \mathrm{~ms}$ \\
Node transmission mode & $t_{T X}$ & $570 \mu \mathrm{s}$ \\
Node receive mode & $t_{R X}$ & $100 \mathrm{~ms}$ \\
\hline
\end{tabular}




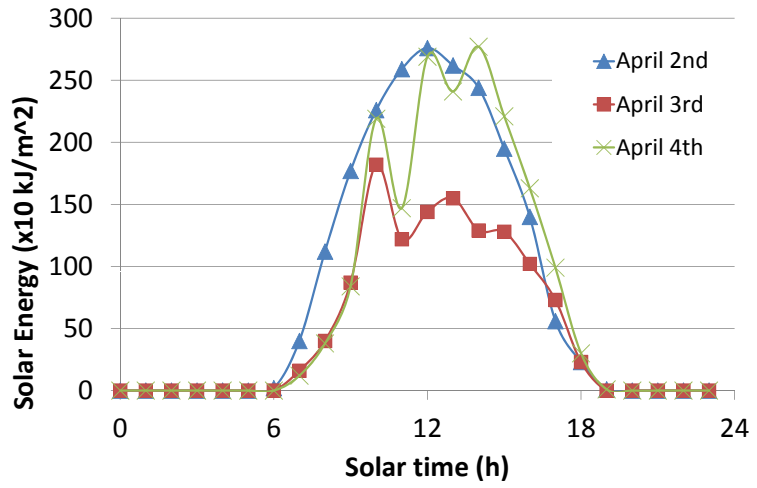

Figure 4.7: Solar energy conditions during the model validation experiments

Figure 4.8 shows a comparison between the real test results and the ns- 3 model estimation. Although some inaccuracies are found, both charge and discharge slopes are very similar. However, charge and discharge cycles seem to be shifted in time. This effect is due to the solar database import process. Since solar data offered by AEMet is the energy integrated during a whole hour, the model uses a constant panel supplied current per hour. Consequently, the sun rises earlier in the simulations and sunset is also delayed.

\subsection{Conclusions}

In this chapter, a new energy model for the ns-3 simulator using super-capacitor energy storage with solar energy-harvesting recharge has been presented. The model is dynamic because it considers radio mode changes and solar radiation variations per hour. This innovation is very important for future network designs because it makes it possible to simulate the impact of radiation variability during several days, weeks, or months. Moreover, solar radiation values can be easily imported from public meteorological databases in different world locations. As an example, this chapter uses the records of the AEMet Spanish office.

The presented experiments are focused on validating the solar panel energy model, the capacitor energy model, and the node consumption model. Results show a good correlation between the simulations and real experiments.

As will be shown in Chapter 5, this model can provide assistance during the design phases by testing different component magnitudes before deploying the network and can also be used aiding in the research of energy-aware policies. 


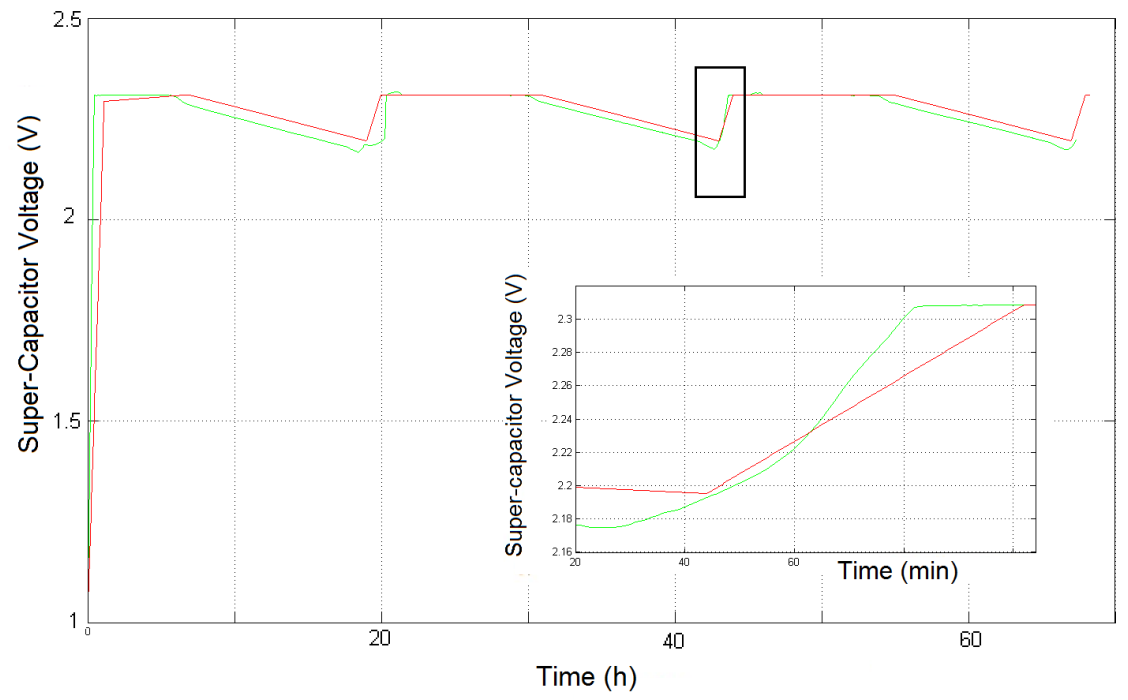

Figure 4.8: Real test results and model estimation 


\section{Chapter 5}

\section{Experimentation}

\footnotetext{
Arefully planning the necessary hardware and algorithms that are going to be utilized is necessary to succeed in the deployment of UWSN. This can obviate node and even network failures once the applications are deployed, which is of extreme importance since retrieving the already deployed nodes can be very expensive and sometimes even impossible.
}

Simulations are a very convenient way of testing algorithms and protocols before their actual deployment. However, one has to be certain that the simulation results are as accurate as possible. The use of, for example, a physical model that is too simple or supposing synchronization among all nodes can alter the behaviour of protocols and sometimes lead to wrong or incomplete conclusions

In this chapter, the utility of the models previously presented is demonstrated by analysing their usefulness in a real monitoring installation scenario in an offshore fish farming facility. A brief description of the scenario is given, followed by a comprehensive study on the performance of the MAC protocols using an underwater wake-up modem. Subsequently, an extensive study of various configurations for energy-harvesting is performed pursuing energy-neutral operations. Finally, both models are employed for planning the deployment of the underwater application.

\subsection{Case Study}

This scenario is part of the project entitled "UWSN ambient monitoring for fish farming facilities". It is a Spanish research project involving an unattended monitoring installation in an offshore fish farming facility. The general architecture is depicted in Figure 5.1, where different sensor nodes are placed near the fish nets and the sea bottom. These nodes are capable of measuring various environmental variables on demand and sending the data to the sink. 


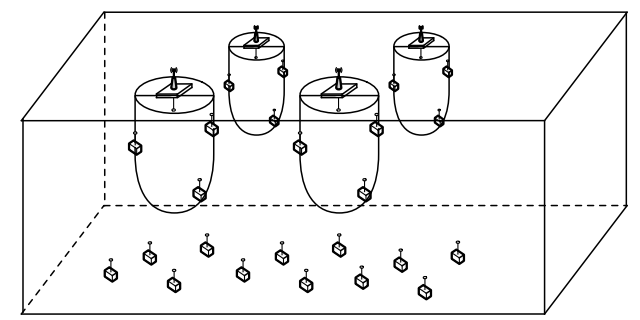

Figure 5.1: Diagram of the simulation scenario

Sink nodes (there might be more than one sink node depending on the installation requirements) are placed on buoys on the sea surface and equipped with solar energy-harvesting capabilities. They also include a radio modem to communicate with an onshore installation.

A sink may require that a group of nodes periodically send some environmental variables. To keep the architecture as flexible as possible, there is no need for these nodes to be known a priori. For example, the sink can send a message asking for certain information and the desired sample frequency. The nodes that are capable of providing this information have to compete to acquire the channel and send the information.

Finally, all nodes are equipped with an underwater acoustic modem with integrated wake-up capabilities like the ones introduced in Chapter 3.

\subsection{Simulation Experiments with MAC Protocols and the Wake-Up Modem}

Given the application constraints of the underwater nodes having to remain operative and unattended over extended periods of time and the network load not being periodically and uniformly distributed, we discarded schedule-based protocols from this study. Moreover, the use of any type of time slots for transmitting data would imply time synchronization, which would increase both the energy communication costs and its complexity.

The FDMA alternative was also abandoned due to the reduced bandwidth of the underwater modems and the fact that previous empirical experiments discarded it [59] [6]. In addition, CDMA, although promising, requires high computational power. Moreover, as it has been previously introduced in Chapter 2, the transmission speed of the different network users decreases considerably with the number of codes. 
Table 5.1: Underwater modem energy consumption

\begin{tabular}{ccc}
\hline \hline MODE & Wake-up & Modem \\
\hline TX mode & $120 \mathrm{~mW}$ & $120 \mathrm{~mW}$ \\
RX mode & $8.1 \mu W$ & $24 \mathrm{~mW}$ \\
IDLE mode & $8.1 \mu W$ & $24 \mathrm{~mW}$ \\
SLEEP mode & - & $3 \mu W$ \\
\hline \hline
\end{tabular}

For the foregoing reasons, random-based protocols that do not need to know the propagation time between nodes, or have any need to synchronize, were found more appropriate. The backoff mechanism is a common feature of these protocols as a contention and collision recovery tool. Hence, it is important to analyse how this mechanism influences the performance of the UWSN MAC protocols. Thus, the specific protocols chosen for this study were ALOHA-CS, MACA, FAMA, and T-Lohi. All four protocols performance is studied with and without the use of acknowledgements. Moreover, FAMA's original specification allows for a burst mode, which is also included in the study.

UWSNs are very energy dependant, which is why a study focusing only on the network performance would not be complete without taking care of the energy consumption. With the exception of T-Lohi, which was specifically developed for UWSNs and which takes advantage of the wake-up mechanism, the remaining protocols under study were not designed to make use of this feature. Nonetheless, they have been adapted to use this energy-saving wake-up mechanism.

\subsubsection{Simulation Parameters}

The Bellhop propagation model was used for the simulations. It was fed with the SSP and bathymetry data, obtained using the WOSS API [71], for the Spanish Mediterranean coast near Burriana where the application is to be deployed. The modem consumption parameters, extracted from [28], are shown in Table 5.1. Finally, the simulations were executed at 500, 1000, 1500, and $2000 \mathrm{bps}$ so the effects of the transmission speed could be analysed.

A scenario comprising an area $100 \times 100$ m was tested with 10 to 100 nodes randomly deployed. Only one sink was placed at the centre of the scenario. Each scenario was simulated several times in order to achieve a confidence interval of \pm 5 with a confidence level of $95 \%$. All simulations were seeded using the number 1330703057, independent replications were performed advancing the run number [66], and the simulation stop time was set to $30 \mathrm{~min}$.

Data payload was set to $60 \mathrm{~B}$ and all traffic was generated by a Poisson distribution with an average packet inter-arrival time of $0.2 \mathrm{~s}$, which ensured that the 


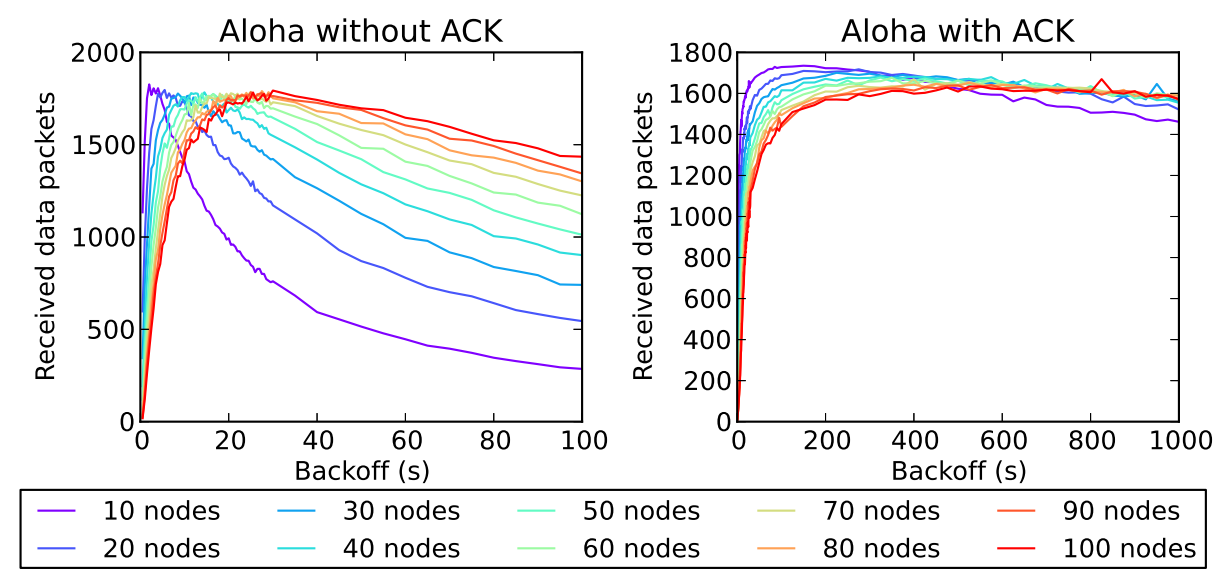

Figure 5.2: Aloha throughput vs backoff analysis

network was working under saturation for all protocols and transmission speed, and directed to the sink node.

\subsubsection{Throughput vs backoff study}

This section aims to find the optimum backoff for the different MAC protocols for various node densities and transmission speeds. The protocols under study were Aloha, FAMA, and MACA. T-Lohi's backoff time is given by the protocol specification and dependent on the maximum propagation time; therefore, it was excluded from this study.

\section{Aloha protocol}

This protocol is the simplest of the implemented MAC protocols in this thesis. Each time there is a packet to be sent the protocol asserts the channel and, if it is free, it begins the transmission. Before sending the actual data packet, a small wake-up signal is sent in order to wake up the receiver. Immediately after this wake-up signal, the actual data packet is sent. However, if the channel is busy, the protocol waits and backs off for a predefined amount of time, after which it restarts the procedure. Packets are kept in a FIFO queue until they are sent. In addition, the same protocol is implemented but including acknowledgements. In this case, packets were kept in the FIFO queue until the acknowledgement was correctly received. 


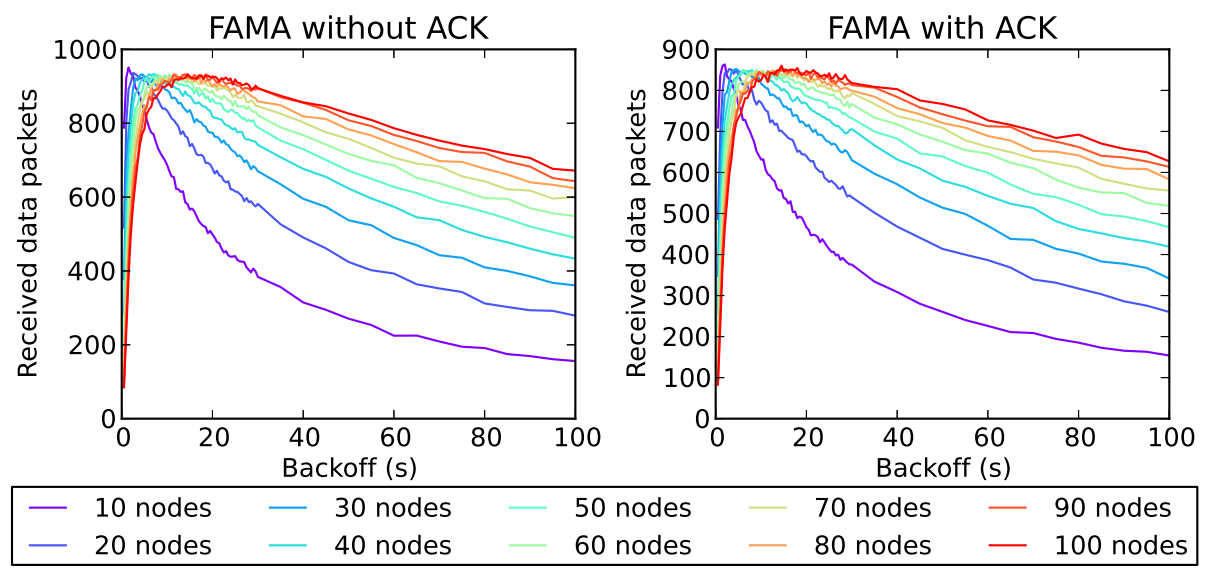

Figure 5.3: FAMA throughput vs backoff analysis

Figure 5.2 shows the protocol throughput for various backoff times and node densities at $1000 \mathrm{bps}$. The results for $500 \mathrm{bps}, 1500 \mathrm{bps}$, and $2000 \mathrm{bps}$ show the same behaviour, and so they are not shown here. Nevertheless, the interested reader can find them in Appendix A. Saturation curves behave as expected for the Aloha protocol without acknowledgement. It can be seen that the optimum backoff time increases with the number of nodes, and that the throughput increases with the backoff time, up to a maximum where it starts to decrease.

On the other hand, when acknowledgements are introduced, the saturation curve increases up to a maximum, stabilizes and then starts to decrease slowly. This behaviour was first demonstrated by Kelly and MacPhee in [100]. They demonstrated that for a general random access scheme with packet retransmission and a given packet generation interval of rate $v>0$, which follows a Poisson distribution, there exists a critical value $v_{c}$ with the property that the number of packets successfully transmitted is finite with probability zero or one according to whether $v<v_{c}$ or $v>v_{c}$.

However, this was demonstrated for an infinite amount of time. In contrast, these simulations have a maximum duration of $1800 \mathrm{~s}$. Hence, as the maximum backoff increases, throughput decreases because if a node randomly chooses a high backoff time it is possible that that node will remain in the backoff state without transmitting any data packet until the end of the simulation. 


\section{FAMA protocol}

In FAMA, before sending a data packet, a RTS/CTS exchange is done between sender and receiver. As specified by the protocol, these packets have to be of a specific length, and act as a busy tone, in order to avoid data packets colliding with other packets. The FAMA protocol is designed in such a way that all nodes must remain awake listening to the channel and updating their timers.

In order to take full advantage of the wake-up capabilities, in the implementation performed for this thesis, a node remained in a low-power state until a packet was sent to it directly. Instead of continuously listening to the channel through the main radio, it takes advantage of the carrier sensing capabilities of the wake-up modem and updates the timers accordingly to the channel state.

The implementation of this protocol also included a FIFO queue and a second version with acknowledgements. Further, in line with the original specifications, a burst mode is supported where, once the node has acquired the channel it can send more than one data packet. This mode is also analysed by sending two data packets when the node acquires the channel instead of one, which we call in this work FAMA X2.

Only saturation curves for the non-bursty mode at 1000 bps are shown in Figure 5.3. The results for $500 \mathrm{bps}, 1500 \mathrm{bps}$, and $2000 \mathrm{bps}$ depict the same behaviour and are not shown here. However, interested readers can find them in Appendix A. In addition, since the curves of our so-called FAMA X2 behave the same but with higher throughput, they are also included in the appendix.

As depicted in Figure 5.3, saturation curves behaved as expected for the FAMA protocol without acknowledgement. It can be seen that the optimum backoff time increases with the number of nodes, and that the throughput increases with the backoff time up to a maximum where it starts to decrease. On the other hand, in contrast to the Aloha protocol with acknowledgements, FAMA with acknowledgements has the same saturation curves as FAMA without acknowledgements because there are no collisions, hence no retransmissions.

\section{MACA-like protocol}

Like the previous protocol, before sending the data packet, an RTS/CTS exchange is done between sender and receiver. These packets include the estimated amount of time that it will take the complete communication. This time is estimated using knowledge of the maximum propagation time and the length of the data packets being transmitted. As in the other protocols, packets are kept in a FIFO queue. We also implemented a second version of the protocol that utilizes acknowledgements. 


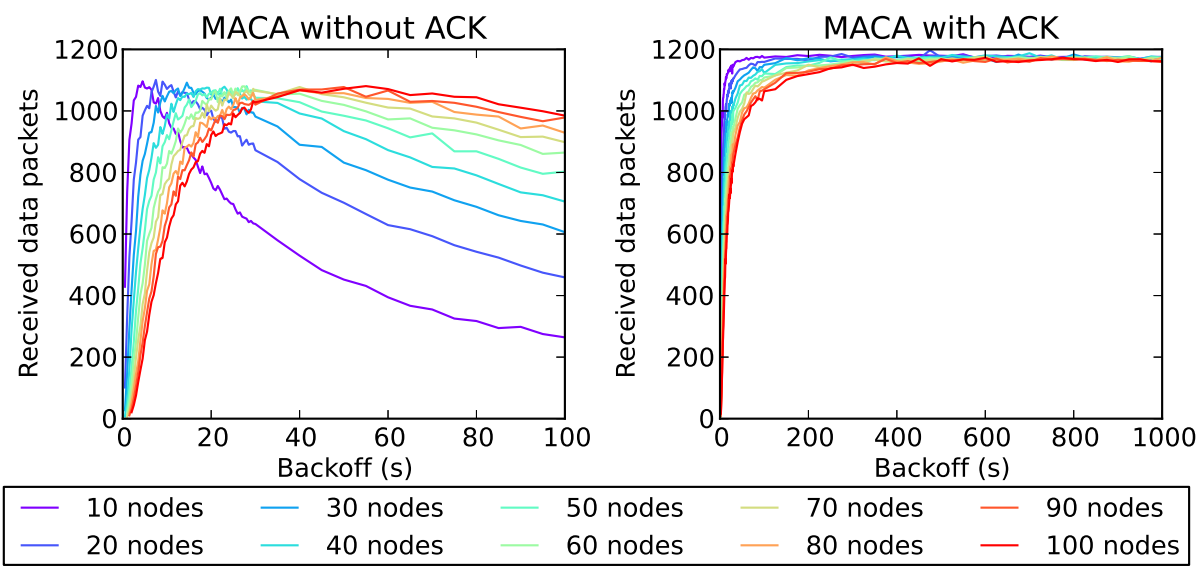

Figure 5.4: MACA throughput vs backoff analysis

Figure 5.4 shows the protocol throughput for various backoff times and node densities at $1000 \mathrm{bps}$. The results for $500 \mathrm{bps}, 1500 \mathrm{bps}$, and $2000 \mathrm{bps}$ depict the same behaviour, and so they are not shown here. However, the interested reader can find them in Appendix A. The saturation curves behave as expected for both versions of the MACA-like protocol, with and without acknowledgements. It can be seen that, without acknowledgements, the optimum backoff time increases with the number of nodes, and that the throughput increases with the backoff time up to a maximum, where it then starts to decrease. On the other hand, with acknowledgements, and because collisions can occur when this protocol is used, and hence retransmissions, the saturation curves behave similar to those of Aloha with acknowledgements.

\subsubsection{Study of delay vs backoff}

This section aims to analyse the effect varying the backoff time has on the delay time. Figure 5.5 shows the Aloha protocol delay with and without acknowledgements for various numbers of nodes and backoff times. The behaviour depicted is also seen in the remaining protocols and is due to network saturation. It can be seen that it stabilizes at approximately $900 \mathrm{~s}$, which is exactly half the simulation time. Because the network is saturated, only the first enqueued packets are transmitted before the simulation ends. Hence, the average packet delay tends to be exactly half the simulation time.

However, it can clearly be seen that for the 10 nodes scenario, the delay decreases with the maximum backoff time. The simulated time is $1800 \mathrm{~s}$ and the maximum 


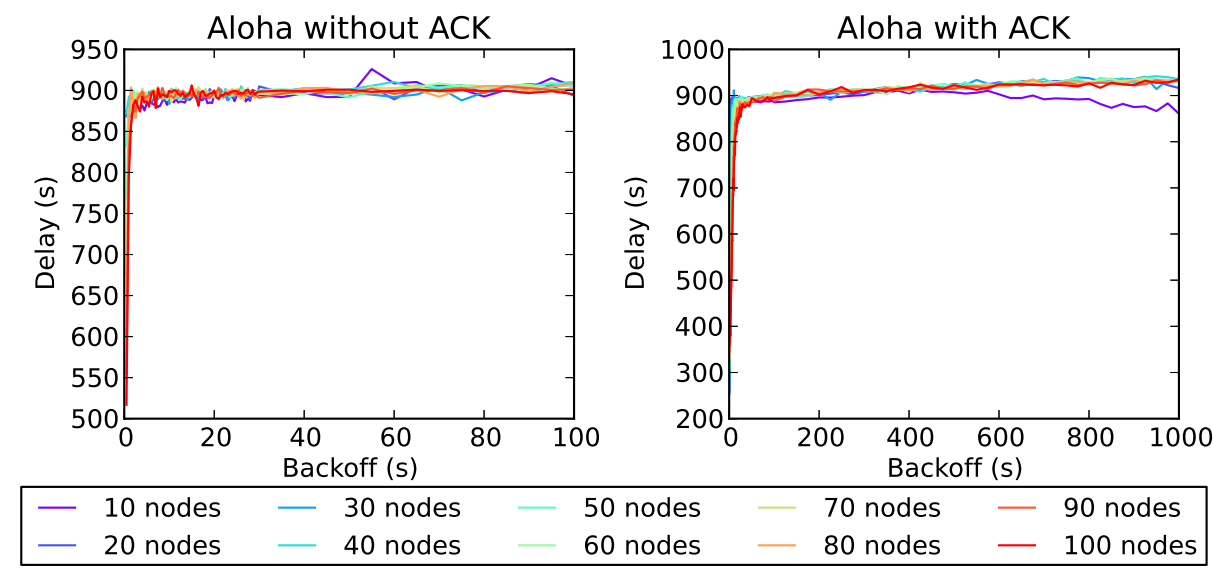

Figure 5.5: Aloha delay vs backoff analysis

backoff time is $1000 \mathrm{~s}$. Hence, if a node randomly chooses a large backoff time, it is possible that that node will remain in the backoff state without transmitting the data packet until the end of the simulation. At the end of the simulation, this effect alters the average delay time rendering these results. Nevertheless, for an arbitrarily long simulation time, the average time when working under saturation is half the simulation time.

\subsubsection{Study of node energy vs backoff}

Energy consumption is also a necessary metric that must be analysed. Figure 5.6 depicts the energy that a node must expend when using the Aloha protocol with and without acknowledgements per data packet correctly received at the sink. The depicted behaviour is also seen in the remaining protocols. It can be seen that the required energy stabilizes to a minimum, which decreases with the number of nodes. It decreases with the number of nodes because the number of data packets correctly received is approximately the same for each case. Hence, the energy expended per node to transmit the same number of packets decreases with the number of nodes.

The figure also shows an increase with the backoff time in the energy expended per correctly transmitted data packet for the 10 node scenario with acknowledgements. The simulation time is $1800 \mathrm{~s}$ and the maximum backoff time is $1000 \mathrm{~s}$. Hence, if a node randomly chooses a high backoff time, it is possible that this node will remain in the backoff state without transmitting the data packet until the end of the simulation. This results in fewer nodes contending for the channel, and so the 


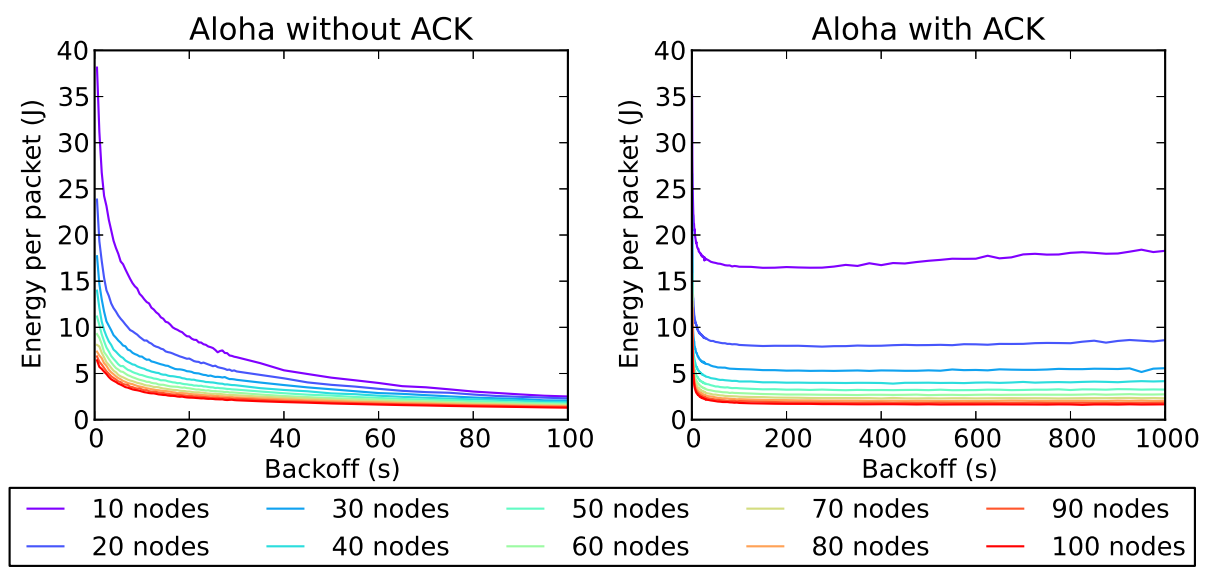

Figure 5.6: Aloha energy vs backoff analysis

energy expended per correctly received data packet increases because the number of packets correctly received is the same.

\subsubsection{Study of sink energy vs backoff}

Figure 5.7 shows the energy that the sink node needs to expend when using the Aloha protocol with and without acknowledgements per data packet correctly received at the sink. The behaviour depicted is also seen in the remaining protocols. As can be seen, the required energy stabilizes to a minimum independent of the number of nodes because the number of packets received is approximately the same regardless of the number of nodes.

\subsubsection{Estimation of optimum backoff}

The results in the previous section show how, once the optimum backoff is reached, energy consumption and delay stabilize. In addition, by closely analysing the saturation curves, the optimum backoff can be calculated depending on the number of contending nodes.

To do this, we employed two methods, one for the general case and the other one for the case with acknowledgements and retransmissions (Aloha and MACA with acknowledgements) since the saturation curves are different.

The general case includes Aloha and MACA without acknowledgements and all versions of FAMA. In Figures 5.2, 5.3, and 5.4, it can be seen how throughput 


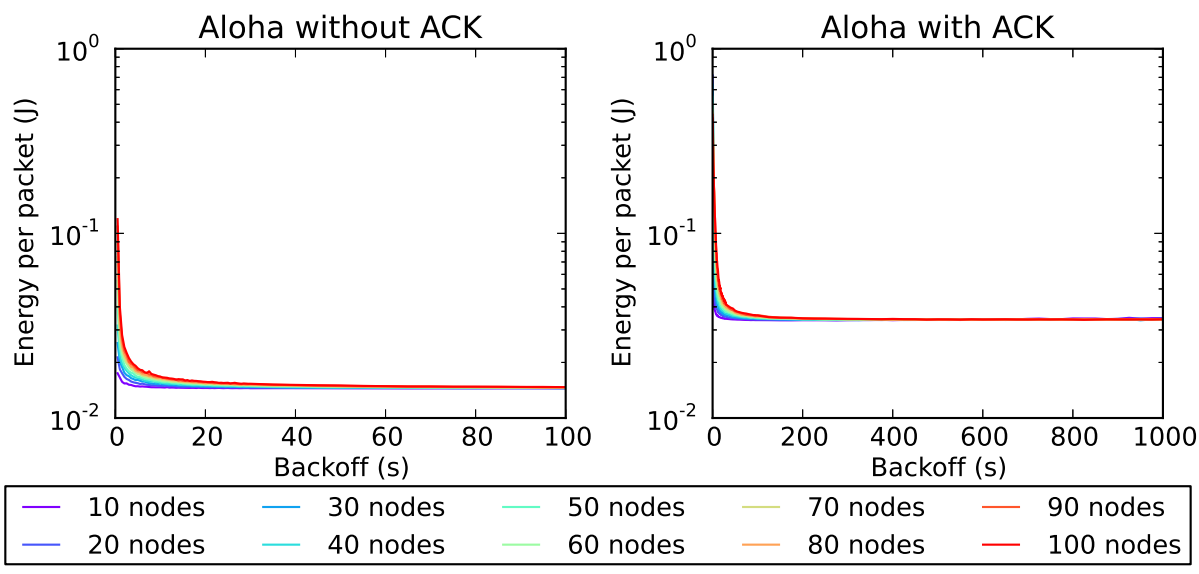

Figure 5.7: Aloha sink energy vs backoff analysis
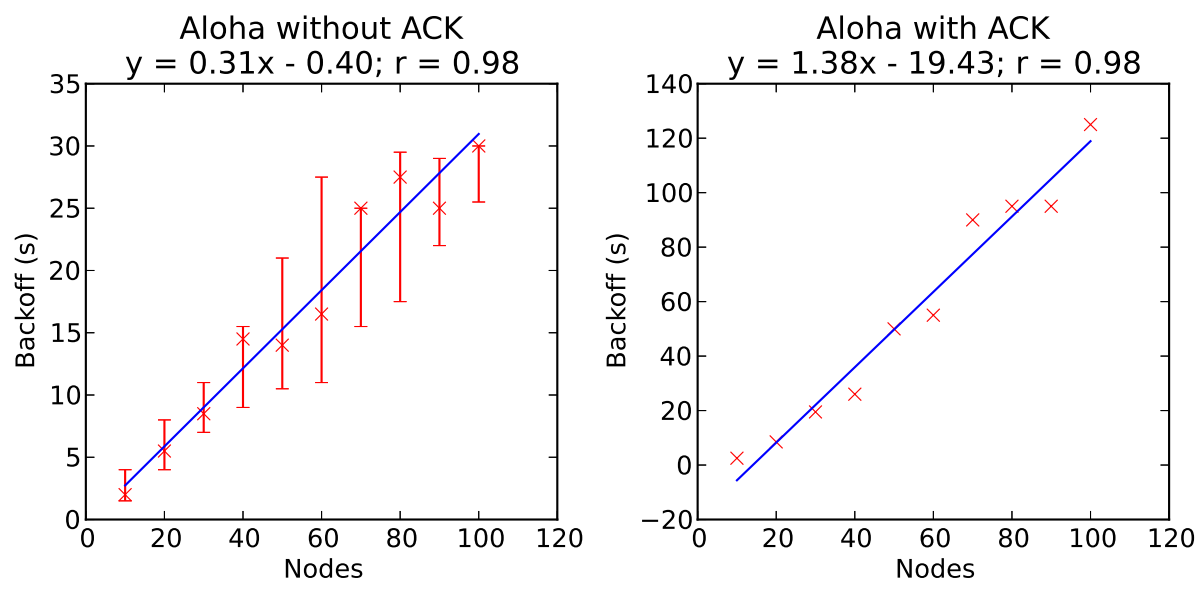

Figure 5.8: Aloha optimum backoff estimation 
increases with backoff up to a maximum and then begins to decrease. Using these points, a linear regression can be calculated for the different protocols, facilitating estimation of the optimum backoff according to the number of contending nodes. The left side of Figure 5.8 shows the calculated linear regression for the Aloha protocol without ACK. The figure includes the range in which, according to the throughput's standard deviation, the backoff time can vary and the maximum throughput still obtained. The figure shows that the linear regression is fairly accurate with a high correlation coefficient " $r$ ".

In addition, on the right side of Figure 5.8, a linear regression of the Aloha protocol with acknowledgements throughput is shown. However, the data for this case, as well as that for the MACA protocol with acknowledgements, are extracted differently. According to [100] and the experimental results, the throughput in this case increases with the backoff time up to a maximum and then stabilizes; thus, the data points used to estimate the optimum backoff are the first ones in which throughput stabilizes, taking into account the standard deviation.

This section shows only the linear regression calculated for the Aloha protocol, the remaining protocols show a similar behaviour with a high correlation coefficient. However, interested readers can find the remaining protocols in Appendix A.

\subsubsection{Influence of acknowledgements on protocol performance}

Having studied how the various protocols behave in terms of throughput, delay, and energy efficiency when the number of nodes and the backoff time are varied, this section investigates the influence of acknowledgements on the MAC protocols. The performance of the Aloha, FAMA, and MACA protocols at their optimum backoff values is closely analysed. Further, we look at how the T-Lohi protocol uses its backoff strategy.

The results shown in this section correspond to the scenarios with a transmission speed of 1000 bps. Interested readers can find the results for 500, 1500, and 2000 bps in Appendix A.

\section{Aloha protocol}

Figure 5.9 shows the throughput, delay, and energy consumption for the Aloha protocol with and without acknowledgements. As expected, the throughput is higher when acknowledgements are not used. In addition, since the network is under saturation, throughput is fairly stable regardless of the number of nodes contending for the channel.

This behaviour explains the energy results seen. The energy expended per correctly received packet at the normal nodes decreases with the number of nodes 

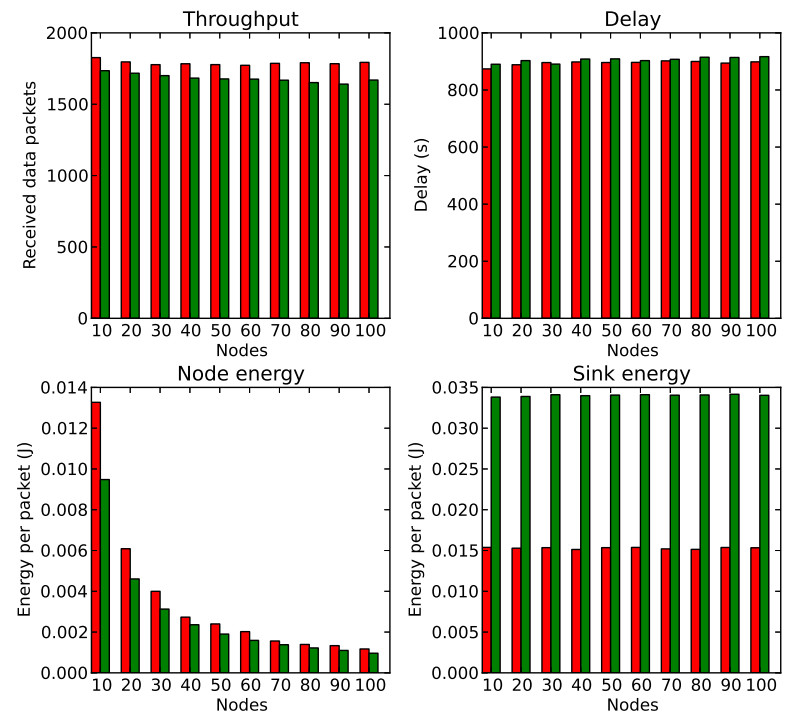

Aloha $\square$ Aloha ACK

Figure 5.9: Comparison of Aloha with and without ACK

because the total number of packets correctly received remains the same. When acknowledgements are introduced, approximately the same amount of data is delivered. However, this mechanism allows for a small energy saving per correctly received packet because the packet delivery ratio increases. On the other hand, acknowledgements introduce a high energy overhead in the sink node, doubling the energy consumption per correctly received data packet.

Finally, as was already shown in the previous sections, packet delay is on average half the simulation time since the network is under saturation. Although it can be clearly seen that, on average, the delay with acknowledgements is a bit higher than without acknowledgements, the standard deviation shows that statistically there are no significant differences.

\section{FAMA protocol}

The throughput, delay, and energy consumption results for all the variants of the FAMA protocol at 1000 bps are shown in Figure 5.10. The throughput is very stable, regardless of the number of contending nodes. As expected, the introduction of acknowledgements incurs overhead which degrades the average throughput. This degradation is higher for the FAMA X2 alternative. When FAMA and FAMA $\mathrm{X} 2$ are compared, the benefit of sending two data packets instead of one in a single 

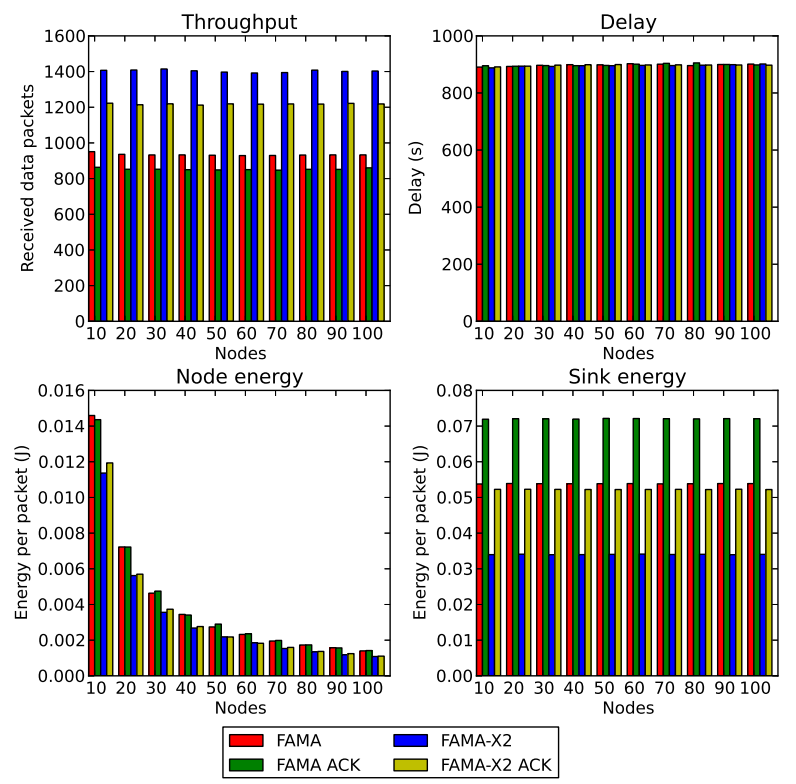

Figure 5.10: Comparison of FAMA with and without ACK

handshake process is very clear, with approximately a $45 \%$ throughput increase without acknowledgements and approximately a $40 \%$ increase when acknowledgements are introduced. The delay, as previously shown in the other sections, is stable and independent of the number of nodes and protocol.

On average, the energy consumption of the nodes is equal for FAMA with and without acknowledgements as well as for FAMA-X2 and there are statistically no significant differences. However, the benefits to be derived from sending two data packets instead of one can be clearly seen when there are few nodes contending for the channel. On the other hand, the overhead of sending acknowledgements is clearly evident in the energy consumption of the sink node. In addition, the benefit of sending two data packets instead of one for the sink energy is also evident.

\section{$M A C A$ protocol}

The results for the MACA protocol performance in terms of throughput, delay, and energy consumption are shown in Figure 5.11. The first thing of note is that throughput is higher when acknowledgements are used. The benefit of including them and assuring correct reception of data packets surpasses their inherent overhead. In addition, packet throughput is independent of the number of nodes 

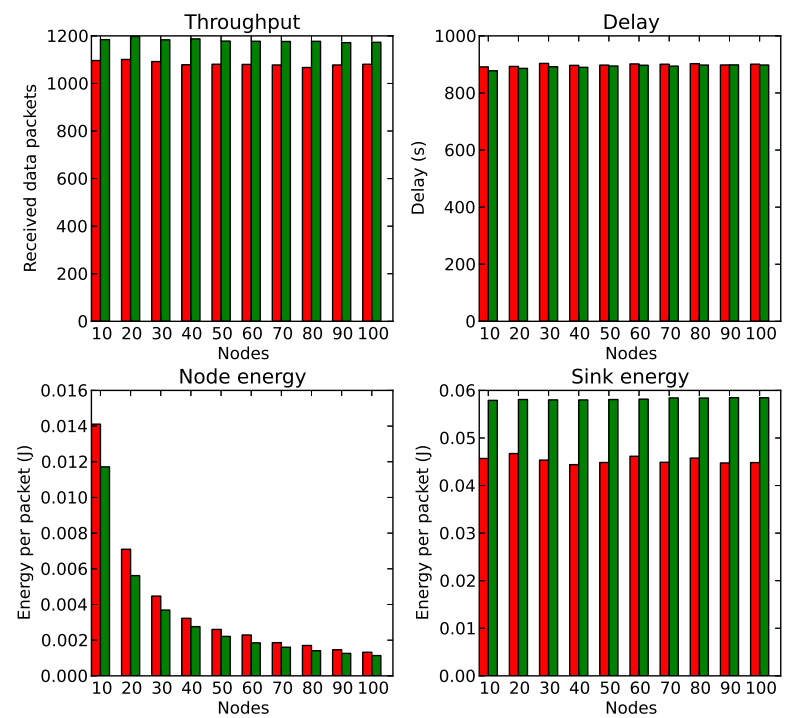

$\square$ MACA $\square$ MACA ACK

Figure 5.11: Comparison of MACA with and without ACK

contending for the channel. On the other hand, delay is stable and independent of the number of nodes, as already seen in the previous sections.

Average node energy consumption per correctly received data packet is higher when acknowledgements are not used due to the low packet delivery ratio. However, this difference tends to decrease as the number of nodes increases because the energy wasted in collisions is divided between more nodes. Moreover, acknowledgements introduce an energy overhead in the sink node, as expected.

\section{T-Lohi protocol}

The results for the T-Lohi protocol at 1000 bps are shown in Figure 5.12. Irrespective of whether acknowledgements are used, T-Lohi's throughput decreases significantly with the number of contending nodes because nodes are continuously backing off. However, as will be shown in the ensuing sections, and can also be seen in Appendix A, T-Lohi's throughput stabilizes for higher transmission speeds, which clearly shows its original design geared towards relatively high speed underwater networks. Nevertheless, as expected, packet delay is stable and independent of the number of nodes and protocol. 

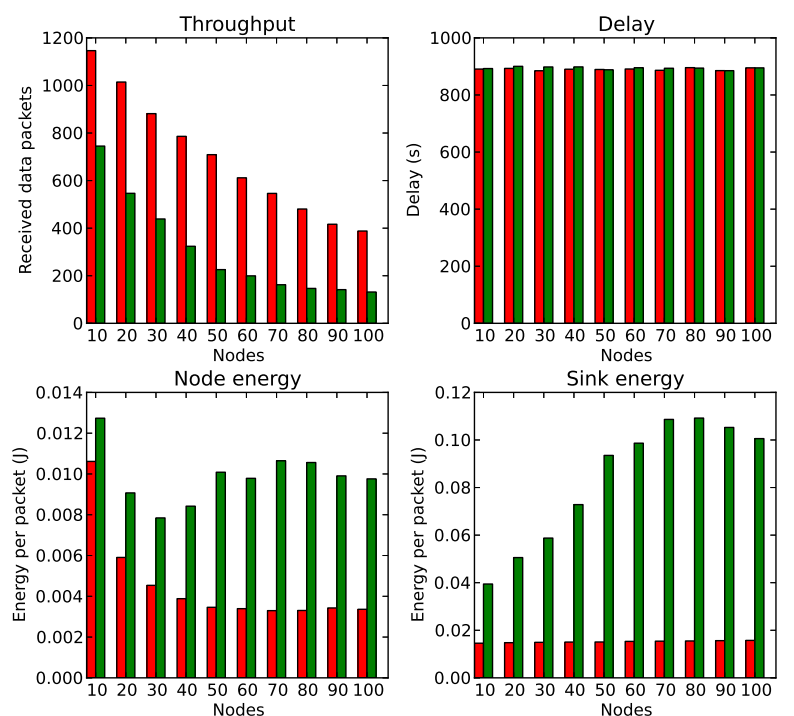

$\square$ T-Lohi $\square$ T-Lohi ACK

Figure 5.12: T-Lohi with and without ACK comparison

Although T-Lohi without acknowledgements shows the same trend in terms of node energy consumption, dividing the total energy expended between all nodes in the network, the continuous backoff and tone transmission imposes an energy overhead for the alternative using acknowledgements. Sink energy consumption per correctly received data packet also shows this. Although this energy consumption is stable regardless of the number of contending nodes for the alternative without acknowledgements, the acknowledgement and tone overhead is clearly in evidence when they are used.

\subsubsection{Influence of transmission speed on protocol performance}

It is obvious that the higher the transmission speed the better a network will perform. Nevertheless, it is advisable to study how this performance increase is affected by the different protocols. To this end, in the remainder of this section, the performance increases of the various protocols when the transmission speed increases is analysed. 


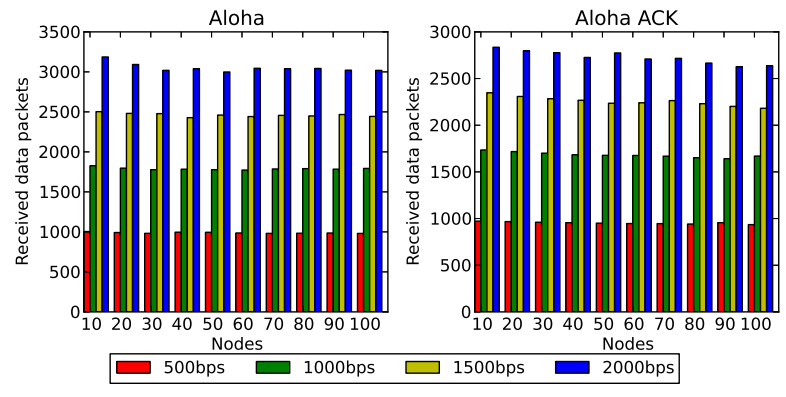

Figure 5.13: Influence of transmission speed on Aloha throughput

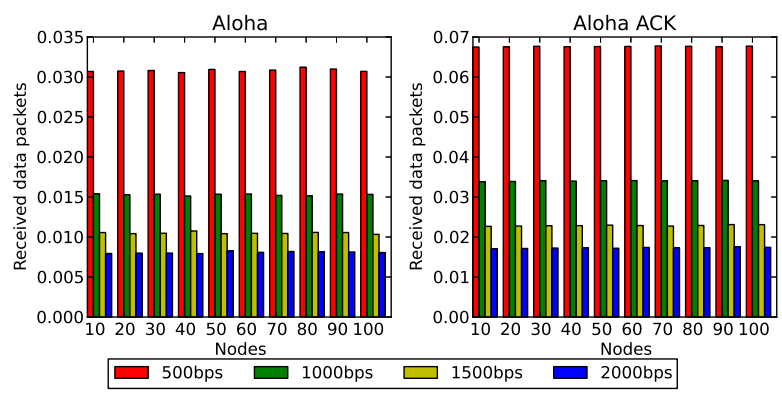

Figure 5.14: Influence of transmission speed on Aloha energy consumption

\section{Aloha protocol}

Figure 5.13 illustrates how throughput varies with various transmission speeds and number of contending nodes for the Aloha protocol. Throughput varies only marginally for one transmission speed and different number of contending nodes, and tends to decrease. When acknowledgements are not used, doubling the transmission speed from 500 bps to 1000 bps increases throughput by approximately $82 \%$ regardless of the number of nodes. However, any further increase in the transmission speed - from 1000 bps to 2000 bps - gives a lower gain in throughput, which also correlates with the number of nodes, $74 \%$ for 10 nodes and $68 \%$ for 100 nodes. As transmission speed increases, the limiting factor for throughput performance changes from the transmission speed to the propagation time.

When acknowledgements are introduced, doubling the transmission speed from 500 to 1000 bps gives a throughput increase of approximately $74 \%, 4 \%$ less than when acknowledgements are not used. Further, increasing the transmission speed from 1000 bps to 2000 bps results in the throughput increasing by $10 \%$ less than the case when acknowledgements are not used, $63 \%$ for 10 nodes and $58 \%$ for 100 nodes. 


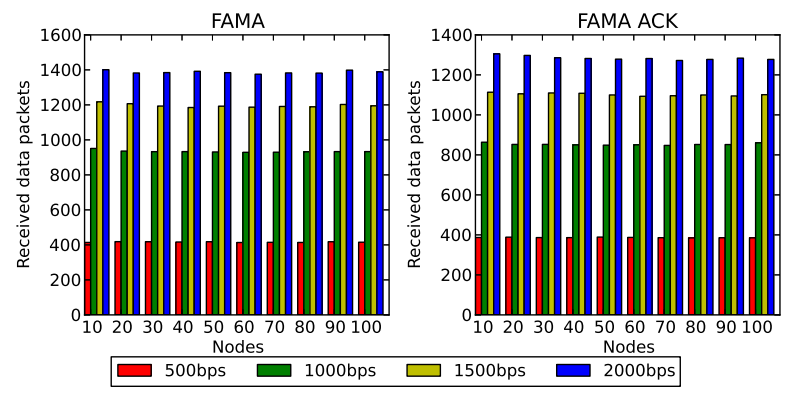

Figure 5.15: Influence of transmission speed on FAMA throughput

Increasing the transmission speed also provides huge energy savings. In underwater acoustic radios, the transmission state is the most energy demanding one and when wake-up mechanisms are used, the main radio remains in the reception state only for the length of time needed to correctly receive the packet. Hence, the percentage of energy spent in transmission increases. This can be clearly seen in Figure 5.14, where the energy expended by the sink node per correctly received data packet is illustrated. The energy expended to correctly receive a packet at $1000 \mathrm{bps}$ is approximately two times higher than the energy needed at $2000 \mathrm{bps}$. At the same time, the energy spent at 1000 bps is half that needed at 500 bps. Results for regular nodes show the same behaviour and can be found in the Appendix A.

\section{FAMA protocol}

Throughput in the FAMA protocol is very stable and there are no statistically significant differences when the number of nodes is varied, as can be seen in Figure 5.15. It can also be seen that there is a significant increment in throughput performance when the transmission speed is doubled from 500 bps to 1000 bps of around $120 \%$ and $130 \%$. However, increasing the transmission speed further from 1000 bps to 2000 bps results in a much lower performance increase of $50 \%$. As transmission speed increases, the limiting factor for throughput performance changes from the transmission speed to the propagation time and, in case of the FAMA protocol, the length of the RTS and CTS packets, which is proportional to the propagation time.

As regards the energy consumption, the conclusions drawn are the same as those for the Aloha protocol. For brevity, these results and the ones showing the performance of the FAMA protocol with a trail of packets are shown in Appendix A. Nevertheless FAMA X2 shows the same behaviour but with higher performance than FAMA. 


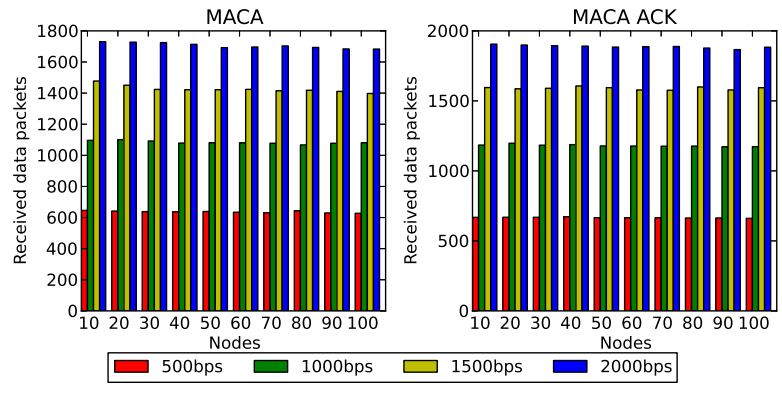

Figure 5.16: Influence of transmission speed in MACA throughput

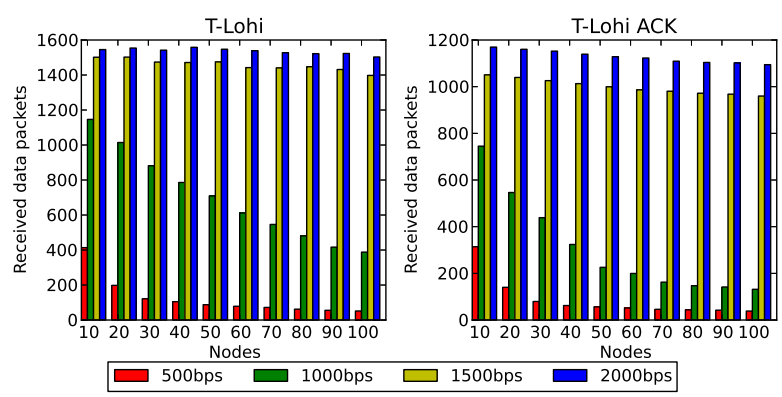

Figure 5.17: Influence of transmission speed on T-Lohi throughput

\section{$M A C A$ protocol}

The MACA protocol also has a very stable throughput performance when the number of nodes is varied, and shows statistically no significant differences, Figure 5.16. Its throughput increases by approximately $70 \%$ when the transmission speed is doubled from 500 bps to 1000 bps and acknowledgements are not used. On the other hand, using acknowledgements allows for an increase in throughput of approximately $77 \%$. Further, increasing the transmission speed from 1000 bps to 2000 bps results in an increase of approximately $60 \%$ in both cases. The conclusions as regards energy consumption are the same as for the previous protocols and are shown in Appendix A.

\section{T-Lohi protocol}

The T-Lohi protocol presents an interesting case. Its throughput is shown in Figure 5.17. Collisions and its backoff mechanism degrade its performance when the number of nodes increases and it is transmitting at relatively low speeds. At 


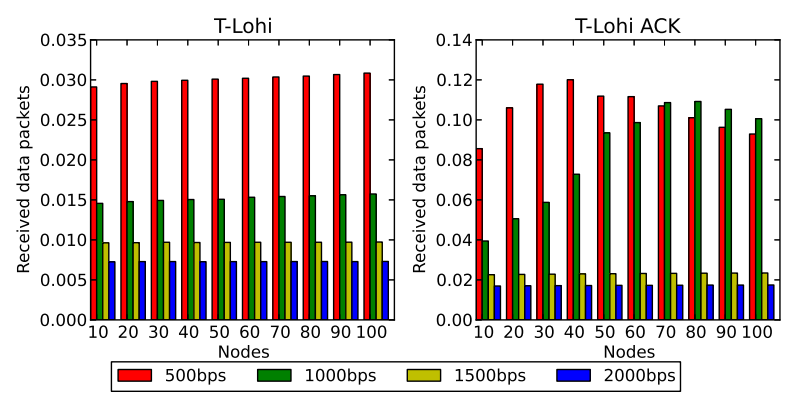

Figure 5.18: Influence of transmission speed on T-Lohi energy consumption

higher transmission speeds, its throughput tends to stabilize although it varies slightly with increasing numbers of contending nodes.

Energy consumption is also affected by the elevated number of collisions. Figure 5.18 shows that the energy consumption of the sink node is as expected when no acknowledgements are used. However, when acknowledgements are introduced, only at high data rates does the energy consumption stabilize and behaves as expected.

\subsubsection{Comparison of protocol performance}

Figure 5.19 compares the protocol throughput for varying number of nodes and transmission speed when no acknowledgements are used. It can be clearly seen that Aloha is the protocol that offers the highest throughput regardless of the transmission speed and number of nodes. Its performance is followed by that of FAMA X2 and MACA. MACA's performance is higher than that of FAMA $\mathrm{X} 2$ for the 500 bps scenario. However, For the remaining scenarios, FAMA X2 achieves slightly better performance. Finally, FAMA and T-Lohi offer the lowest throughput; in the case of T-Lohi, this is because its performance is greatly affected by the number of nodes at lower transmission speeds.

When acknowledgements are used, as depicted in Figure 5.20, Aloha still offers the highest throughput. Its performance is followed by that of FAMA-X2 and MACA. As in the case without acknowledgements, T-Lohi's throughput is severely affected by the number of nodes at lower transmission speeds. However, as the bitrate increases, its performance stabilizes, and it achieves better results than FAMA.

The energy consumption of the regular nodes is depicted in Figure 5.21. T-Lohi's energy efficiency greatly improves with the transmission speed, from being the worst to being the one that offers the best energy consumption. At low trans- 

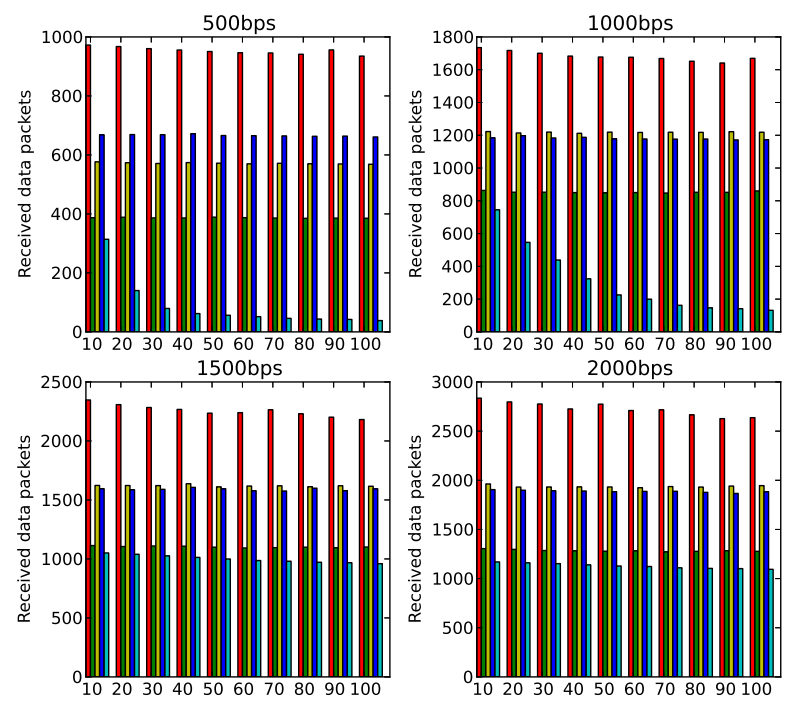

$\square$ Aloha $\square$ FAMA $\square$ FAMA-X2 $\square$ MACA $\square$ T-Lohi

Figure 5.19: Comparison of protocol throughput without acknowledgements
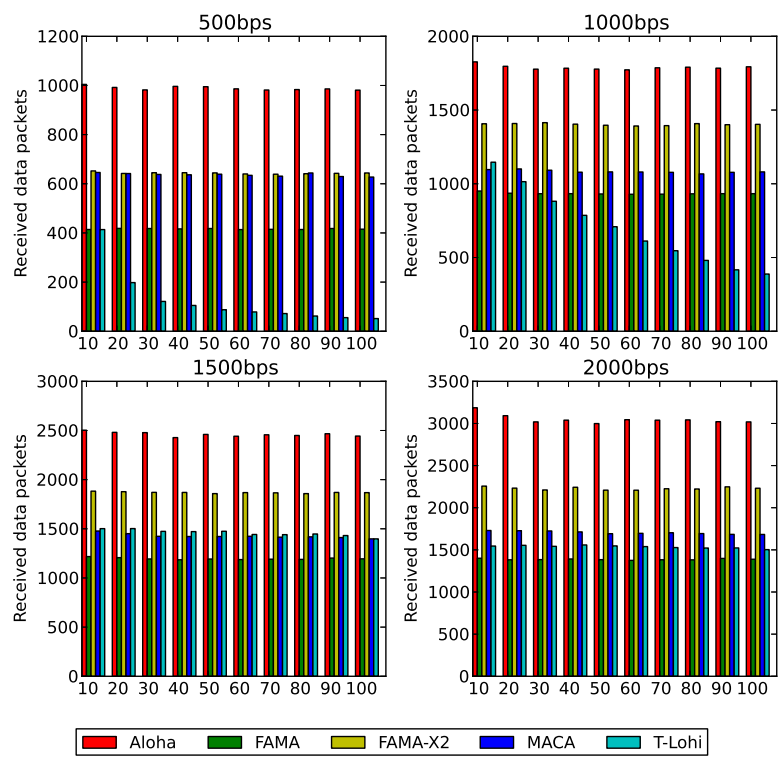

Figure 5.20: Comparison of protocol throughput with acknowledgements 

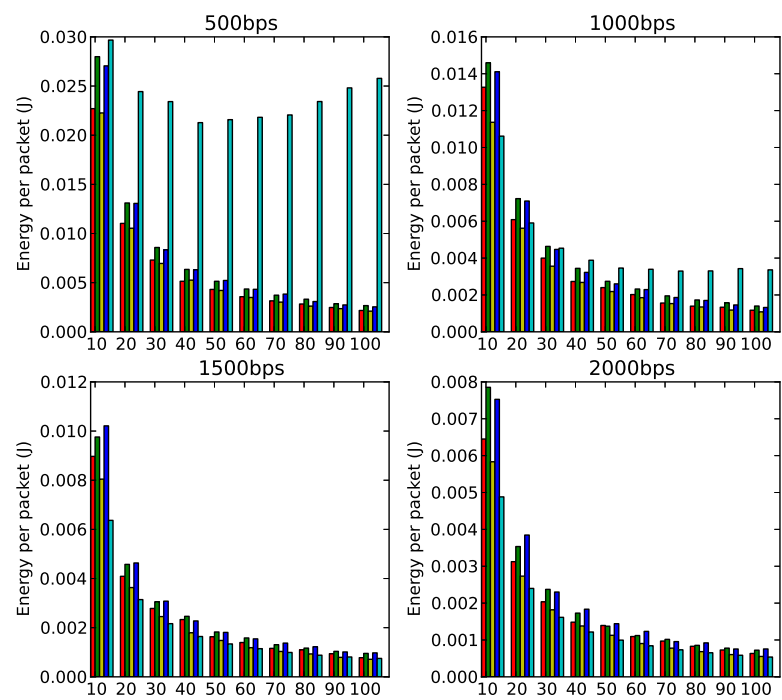

$\square$ Aloha $\square$ FAMA $\square$ FAMA-X2 $\square$ MACA $\square$ T-Lohi

Figure 5.21: Comparison of protocol energy without acknowledgements

mission speeds, FAMA X2 offers the lowest consumption, followed very closely by Aloha.

When acknowledgements are used, as shown in Figure 5.22, Aloha is the most efficient protocol. Its performance is followed by that of FAMA X2 and MACA with almost no difference for high transmission speeds and a large number of nodes. T-Lohi's energy consumption significantly improves with the transmission speed, outperforming FAMA X2 at 1500 bps and 2000 bps with low node density.

Finally, Figure 5.23 shows the energy consumption of the sink node when acknowledgements are not used. As expected, FAMA and MACA have the highest energy consumption since they have to send CTS messages back to the normal node. T-Lohi and Aloha offer the lowest energy consumption, being the last one outperformed by T-Lohi as the transmission speed increases.

Introduction of acknowledgements does not change protocol behaviour in terms of energy consumption of the sink node, as shown in Figure 5.24. However, TLohi is greatly affected by collisions and retransmissions for 500 bps and 1000 bps. Nevertheless, as the transmission speed increases, the performance of T-Lohi stabilizes and its energy consumption matches that of the Aloha protocol. 

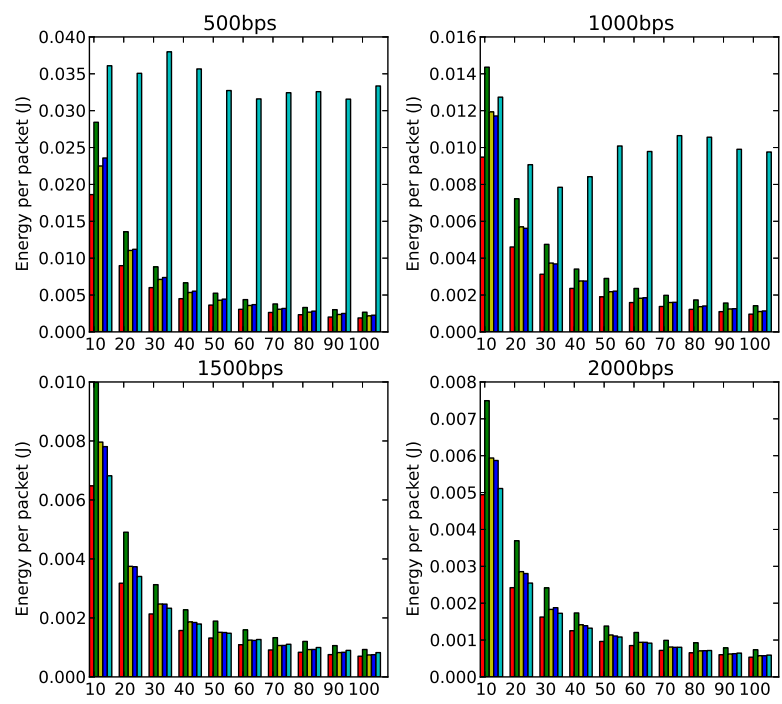

Aloha $\square$ FAMA $\square$ FAMA-X2 $\square$ MACA $\square$ T-Lohi

Figure 5.22: Comparison of protocol energy with acknowledgements
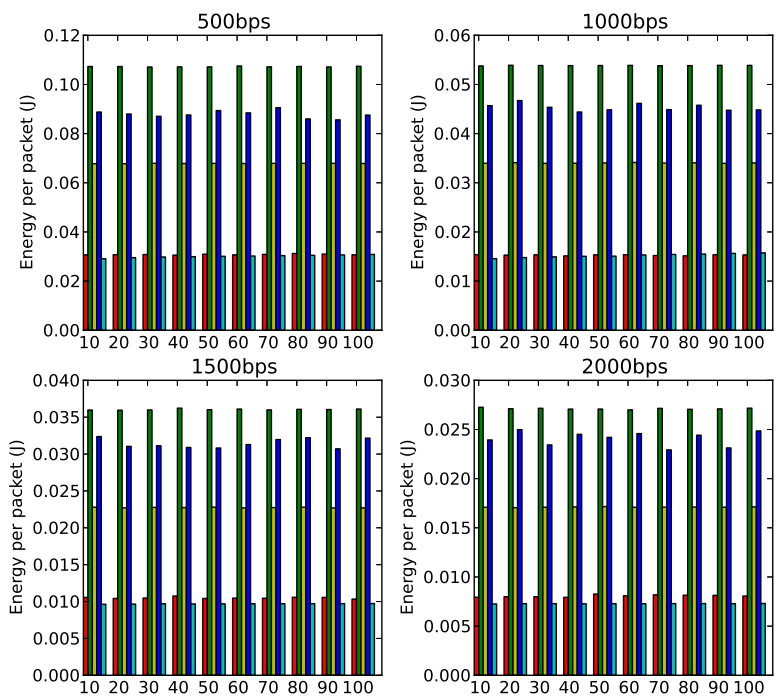

Aloha $\square$ FAMA $\square$ FAMA-X2 $\square$ MACA $\square$ T-Lohi

Figure 5.23: Comparison of protocol energy sink without acknowledgements 

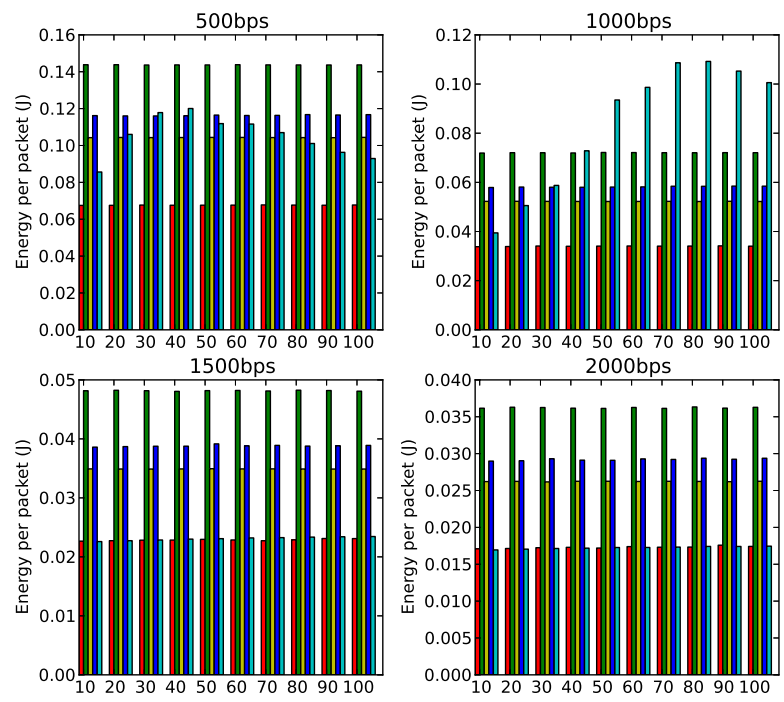

$\square$ Aloha $\square$ FAMA $\square$ FAMA-X2 $\square$ MACA $\square$ T-Lohi

Figure 5.24: Comparison of protocol energy sink with acknowledgements

\subsubsection{Conclusions}

This section investigated the suitability of the various MAC protocols for a real underwater sensor network application. To this end, various MAC proposals were analysed and compared in terms of correctly received packets, energy consumption, and packet delay. The protocols were selected and implemented bearing in mind the hardware characteristics.

In order to perform accurate simulations, real SSP and bathymetry data from the location where the application will be deployed were used, as well as real energy consumption parameters measured from the actual hardware. Further, models such as the Bellhop propagation model and the modem model introduced in Chapter 3 were used.

First of all, a study of the optimum backoff was conducted, with the results showing that it is possible to predict the optimum time according to the number of simultaneous nodes accessing the channel. With this optimum backoff time, we conducted several other studies and, from the simulation results it could be seen how in UWSN a simple approach like Aloha can outperform other more complex alternatives. 


\subsection{Simulation Experiments and Results Using the Energy-Harvesting Module}

Before simulating complex energy-aware protocols, there is a need to predict the maximum current consumption that can be demanded by a network node. The objective is to define energy-neutral strategies during energy-available periods and energy-saving strategies during periods when no energy is available. As an example, several scenarios are proposed in this section to compare energy consumptions. The aim is to show how the proposed model can assist in the discovery of energy depletion conditions and possible unrecoverable energy node failures - and so improve communications reliability.

To perform this evaluation a basic scenario with the following statements is set:

(i) Network star topology. Sensor nodes communicate to a single node that acts as network coordinator and packet sink.

(ii) No failures in the network coordinator power-supply. The rest of the sensor nodes are powered using the energy harvesting system presented in Chapter 4.

(iii) ALOHA MAC protocol. Although throughput can be severely restricted, channel access time is deterministic. No ACK messages are sent in order to minimize network traffic.

(iv) Sensor measurement frequency is configurable in each node.

(v) Nodes can transmit and receive information from the coordinator. For that purpose, sensor nodes will listen to the channel in the duty cycle waiting for incoming packets.

The duty cycle is the same as the one described in Section 4.3.2 and depicted in Figure 4.5. If not stated differently, the simulation parameters for these experiments are the following: time for one packet transmission, 0.57 milliseconds; time that a node remains in the idle/RX state, 102.5 milliseconds; time that a node remains in the sleep state, 60 seconds; and time for sensing, $44 \mathrm{~ms}$. Capacitor capacitance was set to 50 farads, its starting voltage to $0 \mathrm{~V}$, and its maximum voltage to $2.3 \mathrm{~V}$. The node can start its operation only if the capacitor voltage is above $1 \mathrm{~V}$. The efficiency of the DC-DC converter was set to $92 \%$. Solar panel maximum current was set to $40 \mathrm{~mA}$ and the simulation start time was set to match 7 a.m. solar time. Solar radiation data corresponds to radiation measured by AEMet at Valencia (Spain) during April 3 2011. Simulation stop time was set to 48 hours. The sensor is only enabled during Sensor Enabled state and during this state the radio remains in sleep mode. 


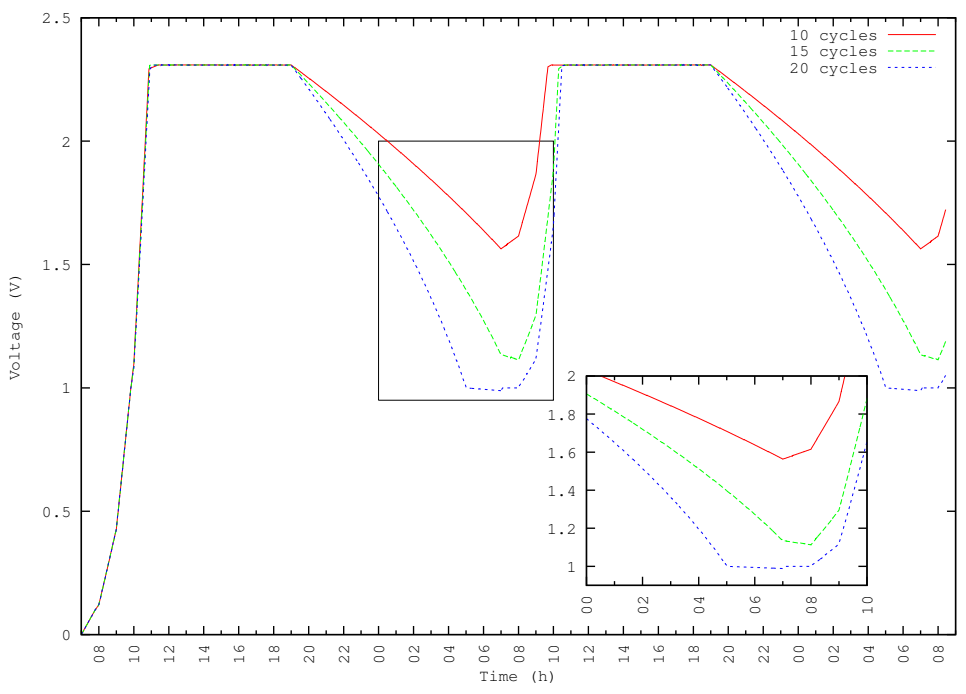

Figure 5.25: TX/RX cycle variation

\subsubsection{Wireless Sensor Network Scaling}

With the aim of understanding how the duty cycle affects the node energy consumption and study the different energy-depletion scenarios (or unrecoverable energy-failures), Figure 5.25 depicts the super-capacitor charge and discharge curves during 48 hours for different TX/RX cycles ( $n$ parameter in Figure 4.5).

At sunrise, charge curves match similarly in the three cases $(\mathrm{n}=10,15$ and 20 cycles). Discharge curves start at sunset and differ for each studied case. A detailed view is shown in Figure 5.25. With 10 and 15 cycles the node never stops its operation since capacitor voltage never falls below one volt, which is the minimum required voltage for the node to maintain operation. At 20 cycles, the energy consumed at night is more than the super-capacitor can provide without recharging - and the node stops operation before sunrise.

At sunrise on the second day, the super-capacitor starts receiving energy from the solar panel between 7 and 8 a.m. When performing at 10 cycles, the supercapacitor is able to recharge while the node is in operation. However, it can be seen how the discharge continues at 15 cycles, although with a much less pronounced slope. A special case is shown in the 20 cycle simulation. In this case, the node is unable to acquire enough energy to start its operation until 8 a.m.

This effect occurs because the energy demands during this hour are greater than the energy provided by the panel, although the super-capacitor is able to recharge, 


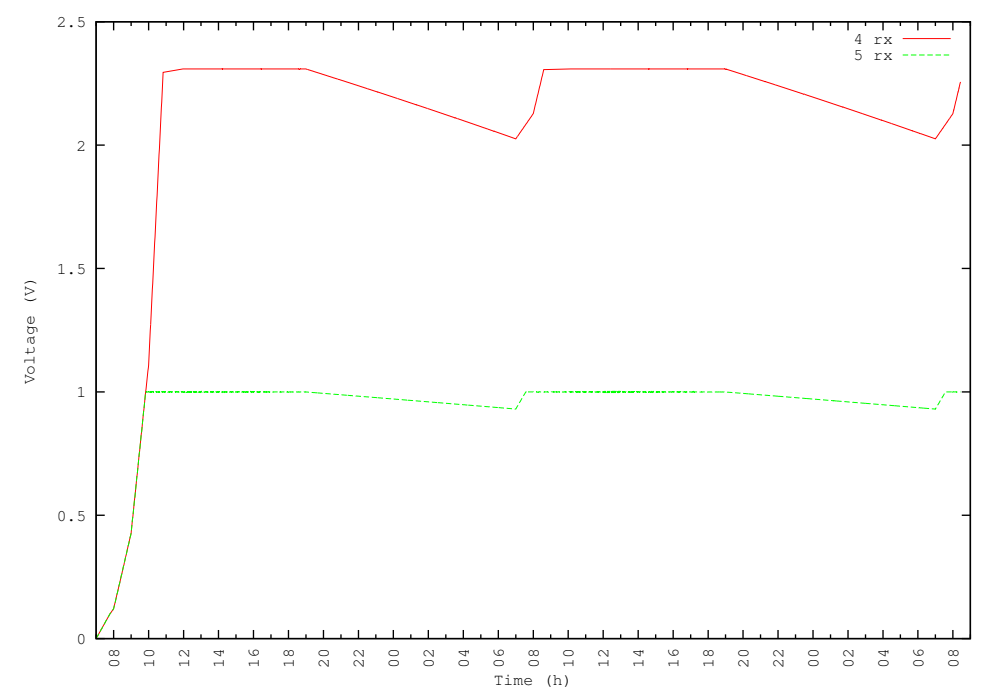

Figure 5.26: Increasing the RX time by $m=4$ and $m=5$ times without hysteresis

the energy is quickly consumed; and consequently, the node is unable to recover operation until 8 a.m. - when the solar panel can provide enough energy.

To study this effect, different benchmarks were performed and shown in Figure 5.26. In this experiment, the duty cycle is modified differently. Instead of varying the $\mathrm{n}$ parameter, which effectively modifies the number of TX/RX cycles performed by the node, the $\mathrm{m}$ parameter is altered. Hence, while maintaining one TX/RX cycle, the RX state will increase its duration by a factor of $\mathrm{m}$. Two different values of $\mathrm{m}$ were chosen to illustrate this experiment: 4 and 5 .

When $m=4$, the node is able to perform as normal. However, when the simulation is configured to $m=5$, the solar panel is never able to provide the node with enough energy to start operation. When the capacitor voltage reaches $1 \mathrm{~V}$, the node starts an operative cycle but the radio RX mode consumes too much energy and decreases the capacitor voltage below $1 \mathrm{~V}$ again, producing an unrecoverable energy node failure.

This problem can be easily solved. The key issue is to store enough energy to carry out a complete cycle before the node becomes active, which can be achieved by including a comparator with hysteresis. The node will stop its operation when voltage falls below $1 \mathrm{~V}$, but it will not become active again until super-capacitor voltage exceeds a certain higher value (a threshold voltage).

To calculate this threshold voltage $\left(V_{\text {tresh }}\right)$ the capacity of the super-capacitor must be taken into account, as well as the total amount of energy consumed 


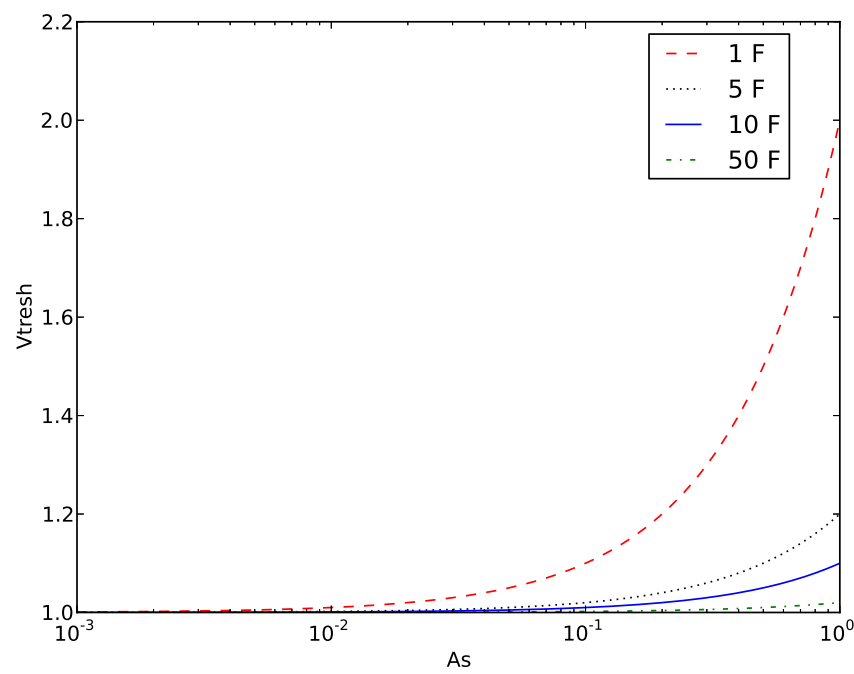

Figure 5.27: Threshold voltage depending on capacitor capacitance and energy consumption

during one cycle. Expression (5.1) calculates this value, and Figure 5.27 plots the voltage threshold for various super-capacitor capacities depending on the amount of energy consumed in one cycle.

$$
V_{\text {Thresh }}=V_{o f f}+\frac{1}{C} \sum_{i} I_{i} t_{i}
$$

Where $C$ is the capacity of the super-capacitor, $V_{\text {off }}$ the minimum voltage at which the node can operate, and the sum represents the total aggregated energy demanded from the super-capacitor during one cycle. This expression has been evaluated for different capacitor capacities and energy consumptions in Figure 5.27.

In Figure 5.28 the results of applying the hysteresis mechanism are shown for $m=5$ and, it can be seen that the node is able to start operation and performs as expected. The hysteresis threshold in this experiment is set at $1.1 \mathrm{~V}$. Although a lower voltage threshold could be used according to Expresion (5.1) and Figure 5.27, 1.1 volts was chosen to provide a security buffer.

Aside from studying the different energy-depletion scenarios and unrecoverable energy failures, simulations can be very useful to decide the order of magnitude of system components for a given application. For example, available energy is 


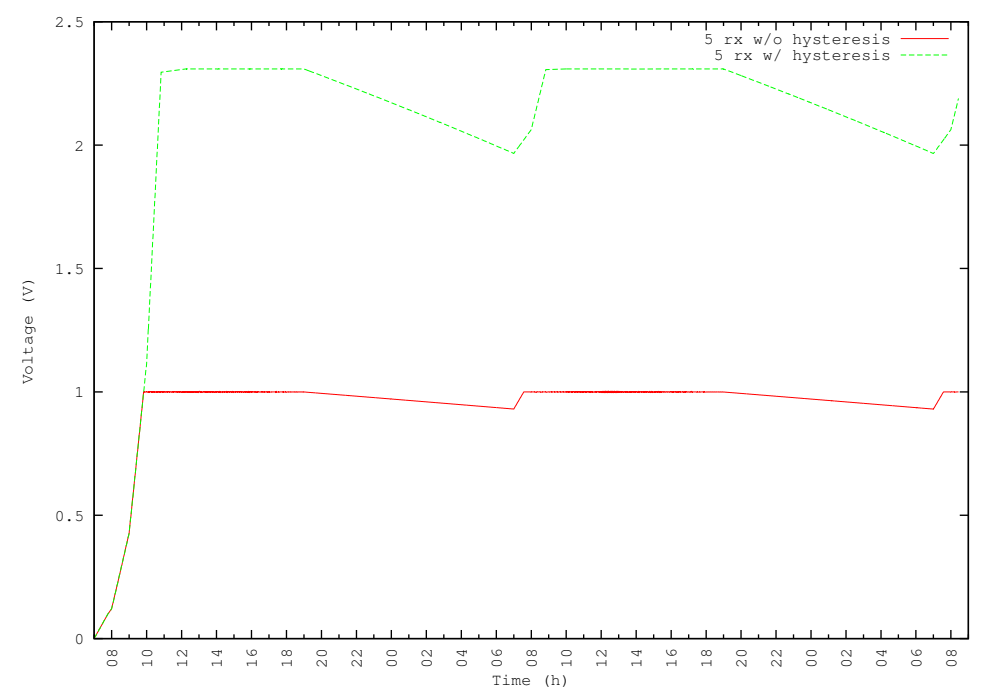

Figure 5.28: Increasing the RX time by $m=5$ times with and without hysteresis

directly proportional to capacitance. In addition, prices are related to this capacitance value, and so making a realistic estimation of the system components is very important when planning the deployment of a WSN.

As an example, Figure 5.29 depicts a comparison between capacitors of 5 and 10 farads. Although our previous experiments were conducted with a 50 farad super-capacitor, it can be observed than using a 10 farad capacitor may suffice. Moreover, different solar panels can be tested via simulation, thereby helping to better tune the hardware nodes to the application requirements. Carrying out this type of study when planning to deploy a WSN can led to important savings in hardware costs.

\subsubsection{Simple Energy Aware Policies}

Previously, it was shown how an unrecoverable energy node failure can be easily solved using a simple mechanism such as hysteresis. In this section, the proposed model is used to show how an energy aware policy can help obtain more statistically meaningful data.

To accomplish this, a one year-long period is going to be simulated in two different locations using solar radiation data from the PANGAEA [95] project. The chosen locations were Tõravere in Estonia during 2011 and Barrow in Alaska (USA) during 2009. Their solar radiation values are depicted in Figure 5.30. 


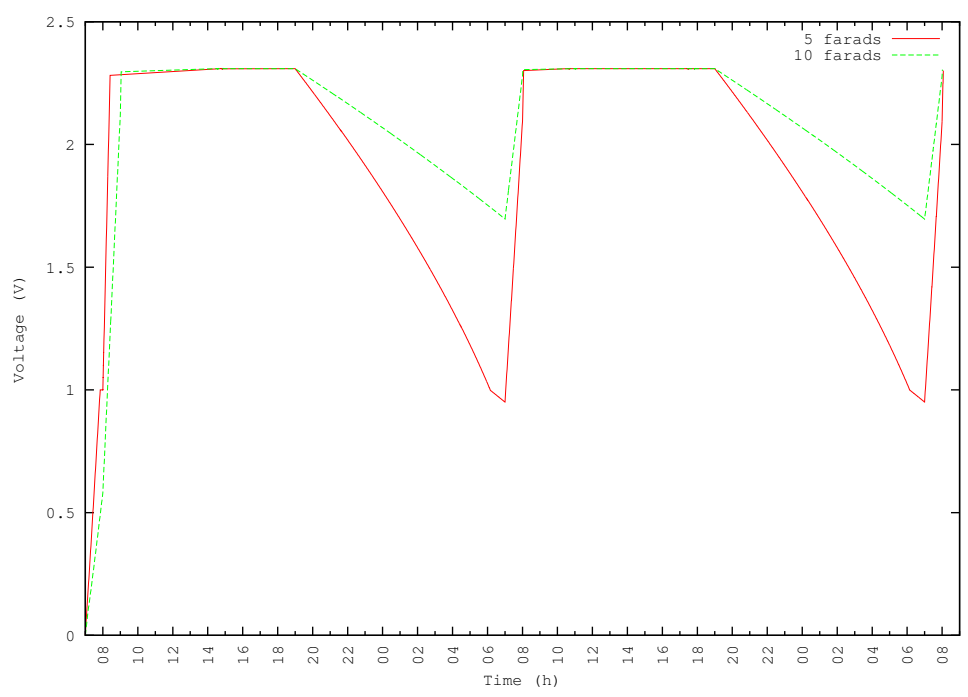

Figure 5.29: Capacitance variation

The study case is as follows. Nodes perform as specified in Figure 4.5; they will only carry out one transmission cycle $(n=0, m=0)$ and their sleep period is five seconds. Power consumption parameters are the same as those specified in Section 5.3.1.

Figure 5.31 depicts the capacitor's maximum and minimum voltage for each day of the year, in the two specified locations. In the Alaska scenario, during the first and the last days of the year, there is not enough solar radiation to charge the capacitor and so the node remains disabled. When the capacitor voltage is higher than 1.1 volt, the node starts its operation and is disconnected below one volt. This means that the days when the minimum voltage is lower than one, but the maximum voltage is higher, the node is disabled for a certain amount of time, usually during the night, without sending data to the sink. This behaviour can lead to several hours passing without information being received, and so isolated islands in the network are created.

Table 5.2, in the "Regular" columns, shows the packet statistics for the two locations compared to the optimum case (in which nodes always have enough energy to send their data). The first thing to notice is that the number of transmissions and receptions in both scenarios is lower than the optimum since there are periods in which the node has not enough energy to operate. As can be seen in Estonia, the mean interarrival time (IAT) between transmissions and receptions is almost $20 \%$ higher than the optimum case - and the standard deviation of the mean in- 


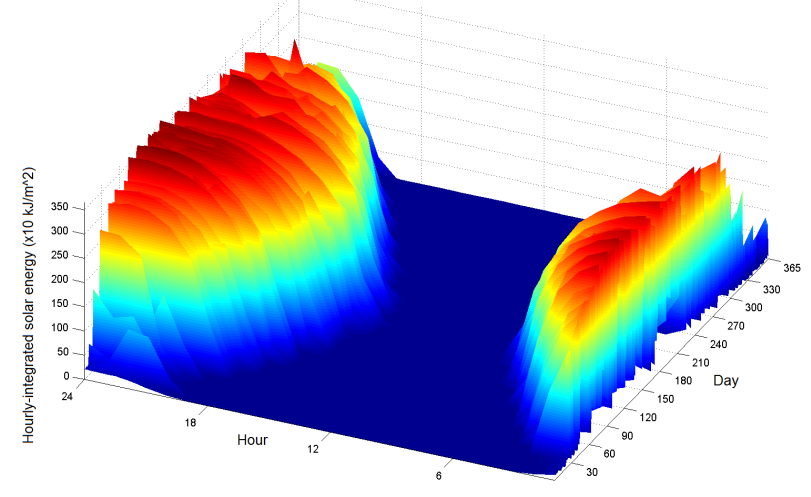

(a) Barrow (Alaska) year 2009

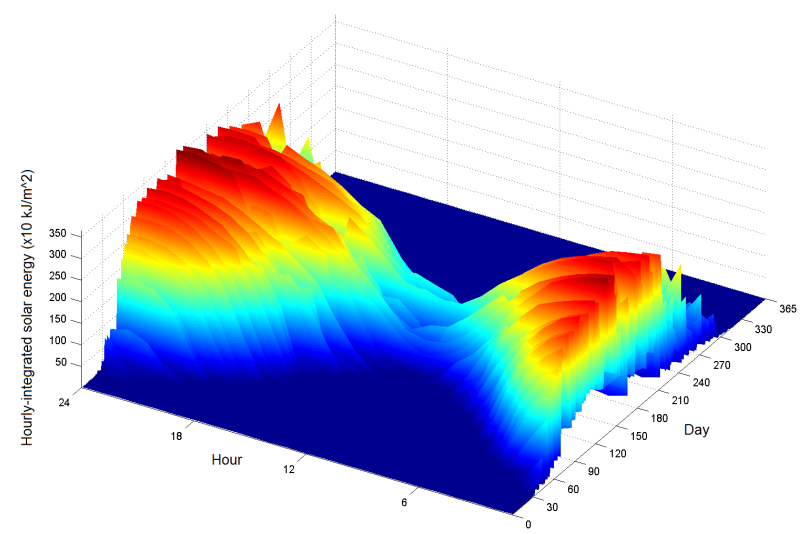

(b) Tõravere (Estonia) year 2011

Figure 5.30: 24 hours, 365 days solar radiation integrated energy (x $10 \frac{\mathrm{kJ}}{\mathrm{m}^{2}}$ ). 0 hours corresponds to maximum solar power time. Day 0 corresponds to January 1 st 


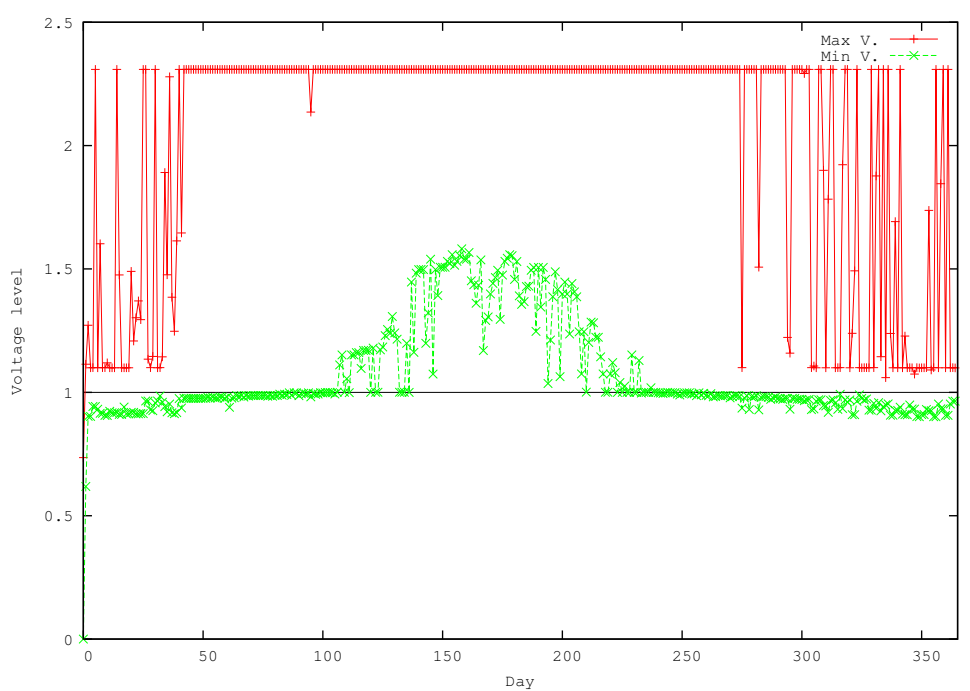

(a) Tõravere (Estonia)

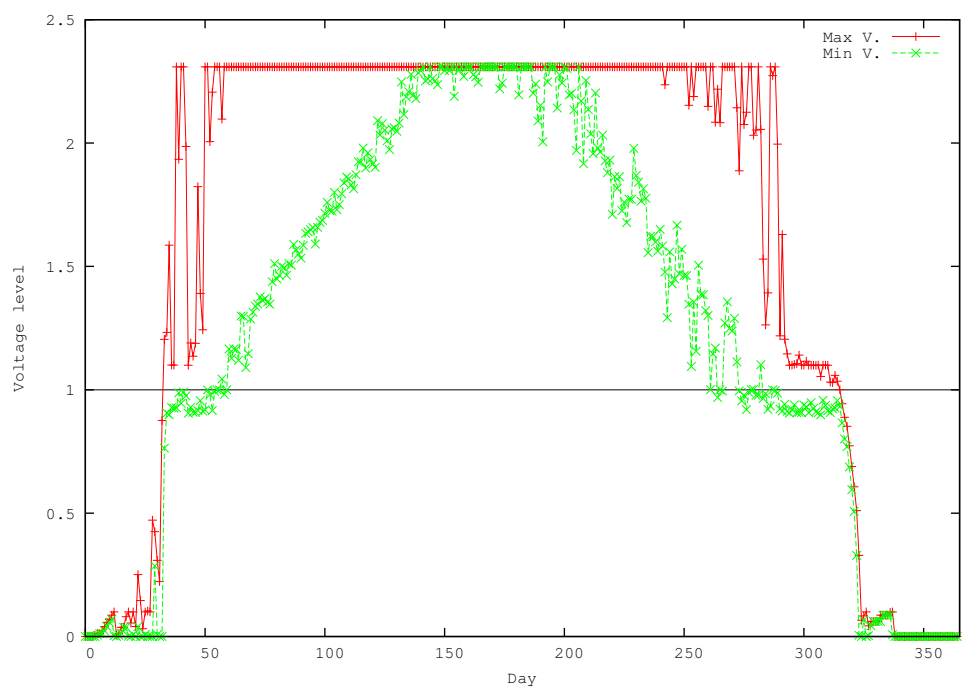

(b) Barrow (Alaska)

Figure 5.31: Node max. and min. voltages during one year 
Table 5.2: Packet statistics with and without energy aware policy in two different locations

\begin{tabular}{lc|cccc|cccc}
\hline & & \multicolumn{4}{|c|}{ Alaska } & \multicolumn{4}{c}{ Estonia } \\
\hline \multirow{2}{*}{ Optimum } & \multicolumn{2}{|c}{ Regular } & \multicolumn{2}{c|}{ Policy } & \multicolumn{2}{c}{ Regular } & \multicolumn{2}{c}{ Policy } \\
& Value & Dev. & Value & Dev. & Value & Dev. & Value & Dev. \\
\hline Total packets & 6127029 & 4051986 & $34 \%$ & 4058898 & $34 \%$ & 5067768 & $17 \%$ & 5049840 & $18 \%$ \\
IAT packets & 5.12 & 5.91 & $15 \%$ & 5.95 & $16 \%$ & 6.19 & $20 \%$ & 6.22 & $21 \%$ \\
STDEV packets & 0 & 231.94 & - & 50.45 & $78 \%$ & 267.69 & - & 58.37 & $78 \%$ \\
\hline
\end{tabular}

terarrival time is also high. This implies no data is received for long periods of time.

To solve this issue, there has been some research effort to design adaptive power management systems [101], [102], [103]. These approaches, although very efficient, are rather complex and might be difficult to implement in a low-power resourceconstrained microcontroller.

Instead, equation (5.2) proposes a simple but effective energy aware policy that modifies the predefined sleep time according to the voltage remaining in the capacitor. This equation only needs to store in memory the previous value of the capacitor voltage - which can be easily obtained using an ADC converter.

$$
S_{t}= \begin{cases}S_{\min } & \text { if } V_{t} / V_{0}>\Theta \\ \max \left(S_{\text {min }}, \frac{S_{t-1}}{\arctan \left(\left(\frac{\Delta V_{t}}{V_{t-1}}+\frac{V_{t}}{V_{0}}\right) \times \alpha\right)} \times \beta\right) & \text { if } \Delta V \geq 0 \\ \min \left(S_{\text {max }}, S_{t-1} \times \arctan \left(\left(\frac{\left|\Delta V_{t}\right|}{V_{t}}+\frac{V_{t}}{V_{0}}\right) \times \alpha\right) \times \beta\right) & \text { if } \Delta V<0\end{cases}
$$

In this equation, $S_{\min }$ and $S_{\max }$ are the minimum and maximum sleep time periods given by the requirements of the application. $S$ is the current sleep time. $V_{0}$ is the maximum current delivered by the capacitor, and $V_{t}$ and $V_{t-1}$ are the current and previous voltages respectively. The constant $\Theta$ defines the voltage threshold value on which the policy will be executed. Finally, $\alpha$ and $\beta$ are two configurable constants that enable an adjustment of the function sensitivity to the application requirements.

By modifying the time a node remains in sleep state (i.e. the duty cycle) this strategy distributes the energy more efficiently and avoids long periods without data collection. Let's suppose a super-capacitor energy-level, and the impossibility of collecting harvested energy (at night for example). Using a good policy, the total number of transmitted packets during this time should be approximately equal to the case when no policy is applied. However, the standard deviation of the interarrival time must considerably decrease. 
Table 5.3: Policy variables values

\begin{tabular}{ll}
\hline Variable & Value \\
\hline$S_{\min }$ & $5 \mathrm{~s}$ \\
$S_{\max }$ & $3600 \mathrm{~s}$ \\
$V_{0}$ & $2.3 \mathrm{~V}$ \\
$\Theta$ & 0.2 \\
$\alpha$ & 10 \\
$\beta$ & 2 \\
\hline
\end{tabular}

The effect of this policy on the simulations can be seen in Figure 5.32, where the parameters of the function were found experimentally and are shown in Table 5.3. In the scenario in Alaska, Figure 5.32b, the policy has no effect on the first and last days of the year since there is not enough energy. But when the node starts working, the policy is able to maintain the node in the working region continuously during more than 300 days compared with 200 days in the scenario without policy.

The same occurs at the scenario in Estonia, Figure 5.32a. When comparing the voltage values for the scenario without the proposed policy, Figure 5.31a, and the policy that implements the policy, Figure $5.32 \mathrm{a}$, it can be seen how by using the policy the node is able to stay in the working region during the entire year.

Table 5.2 includes the packet statistics for the scenarios using the proposed policy. It can be seen that the policy extends the node working time and distributes the transmissions more efficiently. As stated before, the total number of transmissions should be approximately the same with or without the policy, so mean interarrival time (IAT) stays constant in any case. The objective of the presented policy is to communicate with the node with certain predictability and avoid long time gaps, which is accomplished by deceasing standard deviation (STDEV) values. In both cases, this value is reduced by around $78 \%$, and so it can be expected that node activity gaps are reduced to one-fourth - with one transmission cycle almost every minute.

The effect of the policy can also be seen in Figure 5.33, where the distribution of periods without messages sent is plotted according to the voltage level of the super-capacitor. Figure 5.33a shows this distribution without using any policy. As can be seen, until the threshold voltage at which the node stops functioning is reached, packets are sent every five seconds. When this threshold voltage is reached, the node stops its operation and waits between 1 and 60 hours, until there is enough power to restart transmission.

In Figure 5.33b, this distribution is depicted when the proposed policy is applied. It can be seen that the maximum interval without sending messages is one hour, which is the configured maximum interval for the policy. Moreover, the configured 


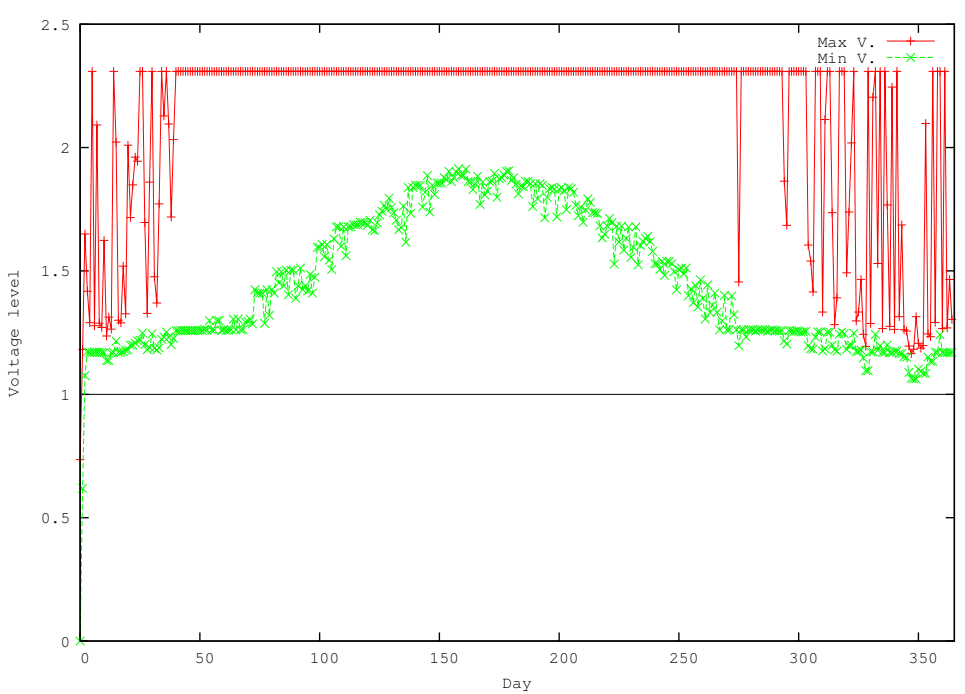

(a) Tõravere (Estonia)

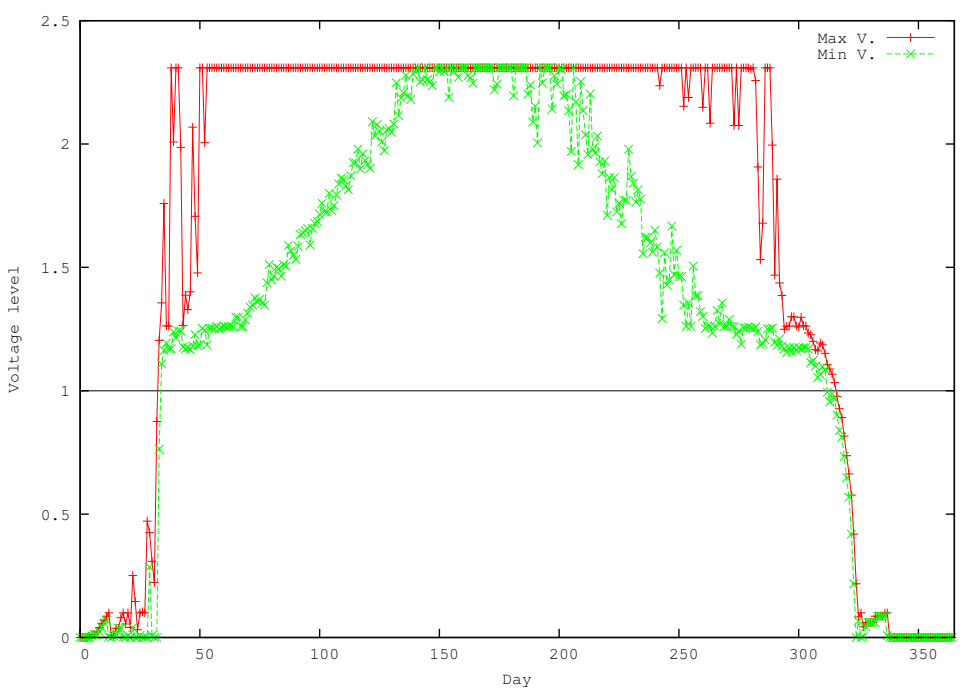

(b) Barrow (Alaska)

Figure 5.32: Node max. and min. voltages when applying the proposed policy during one year 


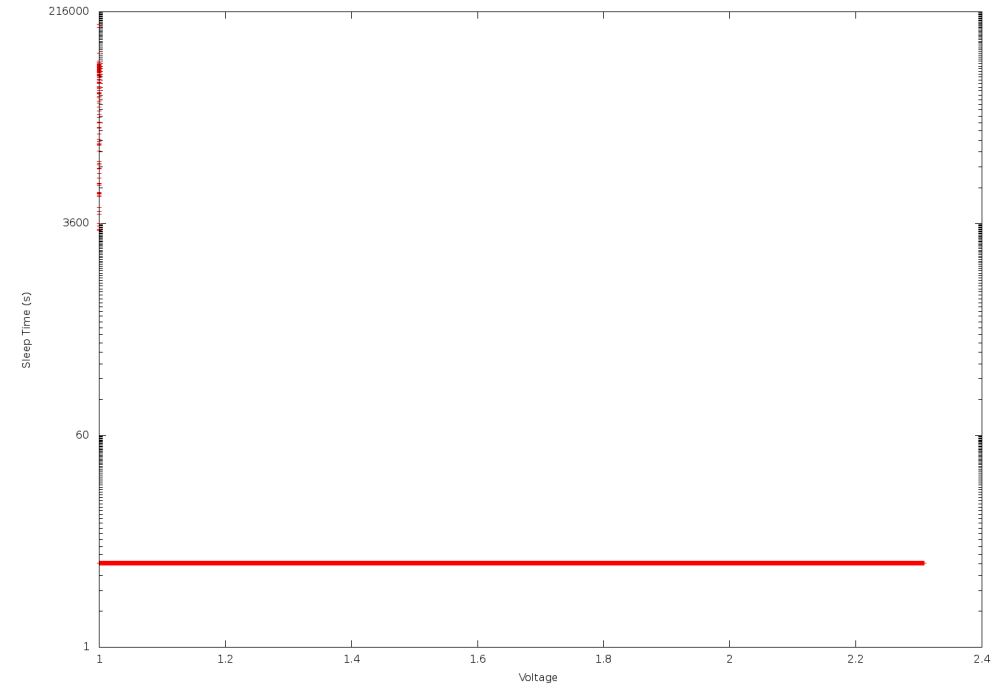

(a) Normal

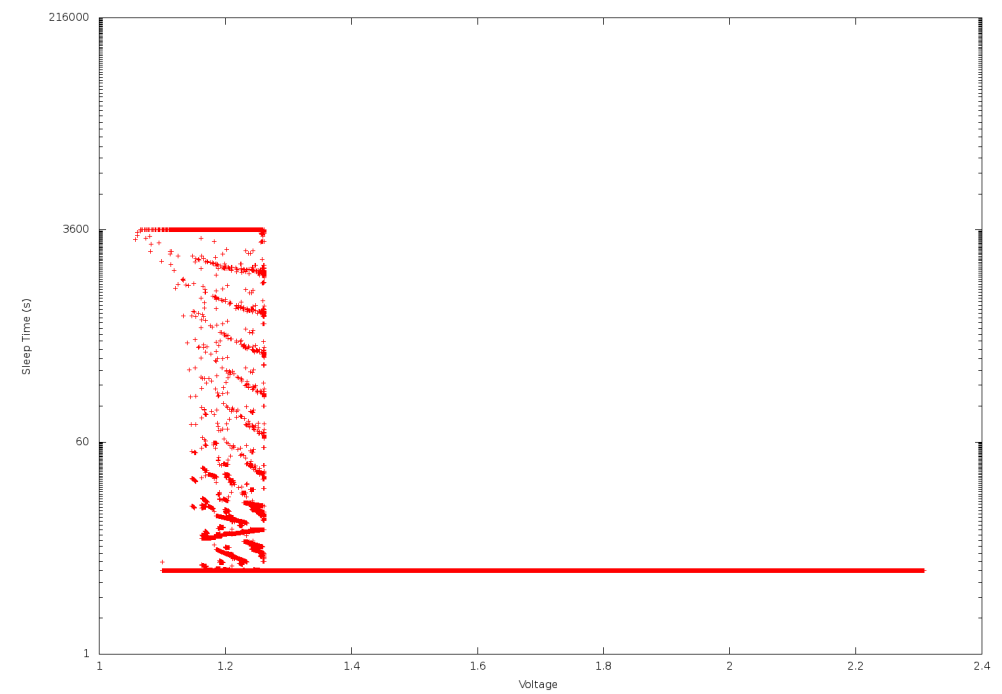

(b) Policy

Figure 5.33: Voltage level and sleep time 
threshold at which the policy starts to be applied can be clearly seen; and it is also possible to see how it is able to distribute the periods without messages in order to reduce the standard deviation of the interarrival time.

In the Alaska case - with no solar radiation during several days - a backup battery is mandatory to guarantee node operation during the whole year. Even when super-capacitor voltage is below $1 \mathrm{~V}$ (node is completely shut down and overall consumption is a few $\mu \mathrm{A}$ ), its energy still decreases until it is depleted. No policy can be applied to extend node life only using super-capacitors. In future work, automatic battery-backup switching will be modelled and evaluated, since the hardware platform implements this feature.

\subsubsection{Conclusions}

This section shows how this energy-harvesting model can provide assistance during the design phases by testing different component magnitudes before deploying the network. Therefore, it opens the possibility of reducing complexity and prices at very early stages of the network design by taking into account the future geographical location.

The experiments conducted reveal the need for intelligent energy control during critical solar periods. In a first approximation, a comparator with hysteresis is introduced - which prevents the operational death of the node. Eventually, an adaptive energy-aware policy has been introduced that enables the operational period of the node to be extended during long and critical solar periods by adjusting the duty-cycle. In an environmental data monitoring application, this can help improve the collected data by avoiding a node total failure and so retrieve more statistically meaningful data.

Generally speaking, this research can assist in the study and development of energy-efficient algorithms that offer energy-neutral operation and dynamic energy adjustment in order to build everlasting wireless sensor networks.

\subsection{Simulation of a Real Underwater Application}

At this point, the underwater model (Chapter 3) and the energy-harvesting model (Chapter 4), have been evaluated and their utility demonstrated separately. In this section, both models are configured to work in conjunction towards the evaluation of a real UWSN application. The aim is to determine which sampling frequency is the minimum that the sink node, which is powered solely by a super-capacitor and recharged with solar energy, can bear without incurring an energy failure. 
This application is part of the Spanish research project entitled "UWSN ambient monitoring for fish farming facilities", already introduced in Section 5.1. Various sensor nodes are placed on the sea floor in order to monitor various parameters required by the application and send them to the sink node, which is on a buoy on the sea surface. The sink node establishes the desired sampling frequency and broadcasts pull messages requesting information to the sensor nodes.

Sensor nodes are equipped with a low-power wake-up system and are battery powered. Conversely, the sink node also has the same low-power wake-up system but is equipped with solar panels in order to harvest energy, storing it in a 100 farads super-capacitor.

The simulation parameters used were the same as those specified in 5.2.1. However, in this scenario, the simulations were done at $1000 \mathrm{bps}$, only scenarios with 10,20 , and 30 nodes were simulated, packet generation was given by sampling frequency, and the simulated time was one year. Solar radiation data was obtained for the year 2011 from Aemet and fed into the simulation.

Given that the traffic pattern is inherently different from that studied in Section 5.2, protocols need to be re-evaluated for these scenarios. However, from the previous results, one can see how FAMA and MACA protocols would be extreme burdens on the energy consumption of the sink. Given that the sink is powered only by a super-capacitor, these two protocols were discarded. This left only the Aloha and T-Lohi protocols, and so they were the ones analysed. Although T-Lohi has a severely limited performance at $1000 \mathrm{bps}$, for low density networks it is still worth studying.

The results of the simulations conducted are shown in Table 5.4. It can clearly be seen that Aloha consumes less sink energy and allows for higher sampling frequency in all scenarios. However, T-Lohi provides a much reliable data transmission with a considerably lower data loss ratio. On the other hand, the delay in the TLohi protocol is considerably higher than that of Aloha. Finally, T-Lohi has a marginally lower energy consumption per correctly received packet for the regular nodes.

Table 5.4: Final scenario simulation results

\begin{tabular}{|c|r|r|r|r|r|r|r|}
\hline Protocol & Nodes & Sampl. Freq. (h) & Sent pkt. & Received pkt. & Lost pkt. (\%) & Delay (s) & Energy (J/pkt) \\
\hline \hline \multirow{3}{*}{ Aloha } & 10 & 2.5 & 25920 & 21942 & $15.35 \%$ & 4.44677 & 0.025 \\
\cline { 2 - 8 } & 20 & 4.5 & 30400 & 25071 & $17.53 \%$ & 8.75827 & 0.017 \\
\cline { 2 - 8 } & 30 & 6 & 34800 & 28047 & $19.41 \%$ & 13.1064 & 0.014 \\
\hline \hline \multirow{3}{*}{ T-Lohi } & 10 & 3 & 21600 & 21313 & $1.33 \%$ & 9.8613 & 0.023 \\
\cline { 2 - 8 } & 20 & 5 & 27360 & 26911 & $1.64 \%$ & 18.9504 & 0.015 \\
\cline { 2 - 8 } & 30 & & 29841 & 29196 & $2.16 \%$ & 27.912 & 0.013 \\
\hline
\end{tabular}





\section{Chapter 6}

\section{Conclusions}

7 He central objective of this thesis was to develop an accurate simulation ecosystem to evaluate UWSN with energy-harvesting capabilities using freely available, high quality, open source tools. Two models were implemented, one of an underwater low-cost, low-power modem with wake-up capabilities and the other of an energy-harvesting circuit with super-capacitor energy storage.

Using these models, we performed a study of several MAC protocols with a view towards the evaluation of a real underwater application. Concomitant with this study, the utility of the energy-harvesting model was shown by analysing how this model can be used to predict energy failures in various parts of the world. Finally, both models were used to simulate a real underwater application and determine its energy requirements.

The most relevant contributions of this work are summarized as follows:

- Review of the basics of the underwater physical layer including currently available modems and in-depth analysis and classification of the most relevant MAC protocols for UWSN.

- Implementation of a model of an underwater modem using the Bellhop model, an accurate underwater channel model. By using the WOSS API, this model can be fed with real SSP, bathymetry, and floor sediment data. In addition, the underwater modem facilitates simulation of an underwater wake-up system based on currently available solutions.

- Implementation of a model of a solar energy-harvesting system with a supercapacitor as an energy storage device. This model can be configured with real radiation data from publicly available databases, facilitating the simulation of several days, weeks, months, or even years. 
- Study of the behaviour of various MAC protocols. The behaviour of the MAC protocols was evaluated for an application which needs a short transmission range and low-power, low-cost devices.

- Study of the energy requirements of an underwater network for monitoring fish farming facilities. Employing the implemented models, the maximum sampling frequency for energy failure was calculated.

\subsection{Future work}

As future work, several lines can be incorporated to further advance this work. Only a reduced subset of the protocols studied in Chapter 2 are implemented in this work. After an in-depth analysis, these protocols were found to best suit the target application needs; consequently, these were the ones analysed. However, other applications might necessitate another approach like, for example, TDMAbased MAC protocol. Hence, more protocols can be implemented to further extend the simulation capabilities.

Some applications might require time synchronization, which is not covered in this work [11]. Correctly implementing synchronization methods in an event-driven simulator is not straightforward because the simulation core only has one clock shared amongst all nodes. Hence, first of all one needs to desynchronise each simulated node. One interesting approach to this de-synchronization can be the one taken by Ferrari et al. in [104].

Once this de-synchronization has been taken care of, various synchronization mechanisms can be implemented. In addition, localization services that usually require synchronization can also be studied [12]. Afterwards, MAC protocols needing synchronization can be accurately evaluated. Moving forward in the protocol stack, there are routing protocols that need localization, and it would also be possible to accurately evaluate them [14].

Regarding the energy-harvesting model, it can be further improved by implementing a battery backup system and further improve the recharge mechanism modelling a charging circuit implementing Maximum Power Point Tracking (MPPT) [105]. In addition, an underwater energy-harvesting system [106] can be modelled to add them to the underwater nodes. 


\title{
6.2 Publications Related to this Thesis
}

The research related to this thesis has resulted in several publications in international journals and conferences which are briefly introduced in the remaining of this section.

\subsubsection{Journals}

This section summarizes the publications in international journals indexed by the Journal Citation Reports (JCR) database.

- S. Climent, J. Capella, N. Meratnia et al., 'Underwater Sensor Networks: A New Energy Efficient and Robust Architecture', Sensors, vol. 12, no. 1, pp. 704-731, 2012.

Abstract The specific characteristics of underwater environments introduce new challenges for networking protocols. In this paper, a specialized architecture for underwater sensor networks (UWSNs) is proposed and evaluated. Experiments are conducted in order to analyze the suitability of this protocol for the subaquatic transmission medium. Moreover, different scheduling techniques are applied to the architecture in order to study their performance. In addition, given the harsh conditions of the underwater medium, different retransmission methods are combined with the scheduling techniques. Finally, simulation results illustrate the performance achievements of the proposed protocol in end-to-end delay, packet delivery ratio and energy consumption, showing that this protocol can be very suitable for the underwater medium.

- S. Climent, J. V. Capella, S. Blanc et al., 'A Proposal for Modeling Real Hardware, Weather and Marine Conditions for Underwater Sensor Networks', Sensors, vol. 13, no. 6, pp. 7454-7471, 2013, ISSN: 1424-8220

\begin{abstract}
Network simulators are useful for researching protocol performance, appraising new hardware capabilities and evaluating real application scenarios. However, these tasks can only be achieved when using accurate models and real parameters that enable the extraction of trustworthy results and conclusions. This paper presents an underwater wireless sensor network ecosystem for the ns-3 simulator. This ecosystem is composed of a new energy-harvesting model and a low-cost, low-power underwater wake-up modem model that, alongside existing models, enables the performance of accurate simulations by providing real weather and marine conditions from the location where the real application is to be deployed.
\end{abstract}


- A. Sanchez, S. Blanc, S. Climent et al., 'SIVEH: Numerical Computing Simulation of Wireless Energy-Harvesting Sensor Nodes', Sensors, vol. 13, no. 9, pp. 11750-11771, 2013

Abstract The paper presents a numerical energy harvesting model for sensor nodes, SIVEH (Simulator I-V for EH), based on I-V hardware tracking. $\mathrm{I}-\mathrm{V}$ tracking is demonstrated to be more accurate than traditional energy modelling techniques when some of the components present different power dissipation at either different operating voltages or drawn currents. SIVEH numerical computing allows fast simulation of long periods of time - days, weeks, months or years - using real solar radiation curves. Moreover, SIVEH modelling has been enhanced with sleep time rate dynamic adjustment, while seeking energy-neutral operation. This paper presents the model description, a functional verification and a critical comparison with the classic energy approach.

- S. Climent, A. Sanchez, S. Blanc et al., 'Wireless sensor network with energy harvesting: modeling and simulation based on a practical architecture using real radiation levels', Concurrency and Computation: Practice and Experience, no. In press, pp. 1-22, 2013

Abstract This paper presents a new energy-harvesting model for a network simulator that implements super-capacitor energy storage with solar energy-harvesting recharge. The model is easily extensible, and other energy harvesting systems, or different energy storages, can be further developed. Moreover, code can be conveniently reused as the implementation is entirely uncoupled from the radio and node models. Real radiation data are obtained from available online databases in order to dynamically calculate super-capacitor charge and discharge. Such novelty enables the evaluation of energy evolution on a network of sensor nodes at various physical world locations and during different seasons. The model is validated against a real and fully working prototype, and good result correlation is shown. Furthermore, various experiments using the ns-3 simulator were conducted, demonstrating the utility of the model in assisting the research and development of the deployment of everlasting wireless sensor networks.

- S. Climet, A. Sanchez, J. V. Capella et al., 'Underwater Acoustic Wireless Sensor Networks: Advances and Future Trends', Sensors, no. In press, pp. 139,2013 


\begin{abstract}
This survey aims to provide a comprehensive overview of the current research on underwater wireless sensor networks, focusing on the lower layers of the communication stack, and envision future trends and challenges. It analyses the current state of the art on the physical, medium access control, and routing layers. It summarizes their security threads and surveys the currently proposed studies. Current envisioned niches for further advances in underwater networks research range from efficient, low-power algorithms and modulations to intelligent, energy-aware routing and medium access control protocols.
\end{abstract}

\title{
6.2.2 International Conferences
}

This section summarizes the publications in international conferences.

- A. Sanchez, S. Climent, S. Blanc et al., 'WSN with energy-harvesting: modeling and simulation based on a practical architecture using real radiation levels', in Proceedings of the 6th ACM workshop on Performance monitoring and measurement of heterogeneous wireless and wired networks - PM2HW2N '11, New York, New York, USA: ACM Press, Oct. 2011, pp. 17-24, ISBN: 9781450309028

Abstract This paper presents a new energy-harvesting provider for ns-3 simulation tool. The provider model assumes solar energy harvesting and super-capacitor storage to supply power to a Wireless Sensor Node. Supercapacitors charge and discharge is dynamically estimated based on both power consumption in the node and solar radiation levels. On one hand, super-capacitors voltage level is refreshed within each radio mode change. However, because a radio mode can be longer than few milliseconds, refreshments are also time schedule. On the other hand, solar radiation data in CSV format (comma-separated values) can be downloaded from public meteorological data bases. The model incorporates these input data being possible to simulate from one minute to weeks or months using real radiation curves observed in a specific world location at a year period. The research presented in this paper will be very useful for the study and development of energy-efficient algorithms with energy-neutral operation to build everlasting Wireless Sensor Networks.

- S. Climent, J. Capella, A. Bonastre et al., 'A new model for the ns-3 simulator of a novel routing protocol applied to underwater WSN', Proceedings of the 2011 International Conference on Wireless Networks (ICWN'11), Las Vegas, NV, USA, pp. 18-21, 2011 
Abstract Providing reliability, scalability and energy efficiency in Underwater Wireless Sensor Networks (UWSN) is very challenging due to its special features. UWSNs usually employ acoustic channels for communications, which compared with radio-frequency channels, allow much lower bandwidths and have several orders of magnitude longer propagation delays. Some routing protocols have been proposed to address these problems, but these approaches usually present unrealistic assumptions or do not consider important questions such as energy consumption or reliability. In this paper we propose the application of EDETA (Energy-efficient aDaptive hiErarchical and robusT Architecture) to the subaquatic acoustic medium. This routing protocol allows a multiple-sink architecture without introducing extra cost. Furthermore, it adds fault tolerant mechanisms to the network structure. An implementation of a subset of EDETA in the ns-3 simulator has been carried out. Extensive simulations have been executed. The results show very high packet delivery ratios with reduced energy consumption while offering scalability and reliability.

- K. Zhang, S. Climent, N. Meratnia et al., 'Practical problems of experimenting with an underwater wireless sensor node platform', in Intelligent Sensors, Sensor Networks and Information Processing (ISSNIP), 2011 Seventh International Conference on, IEEE, 2011, pp. 365-370

Abstract Although in recent years many research activities have been carried out on underwater communication, still many challenges need to be tackled to make the underwater communication suitable for real applications. To this end, availability of an open and well-designed platform and performing real-world experiments are crucial. In reality, the real-world experiments have proved to be much more difficult than expected as the underwater communication channel has not yet fully and precisely been modelled and the performance of signal processing part might be varied from application to application. The deployment itself, online debugging, dealing with variable channel characteristics, and packaging are issues making real-life underwater communication experiments challenging. In this paper, we present initial results of dealing with and solving some practical problems of experimenting with an underwater wireless sensor node platform. We also highlight the drawbacks of current underwater research platform as far as deployments are concerned.

- S. Climent, N. Meratnia and J. V. Capella, 'Impact analysis of different scheduling and retransmission techniques on an underwater routing protocol', in Proceedings of the Sixth ACM International Workshop on Underwater Networks, ACM, 2011, p. 8 


\begin{abstract}
Despite many advances in the area of Underwater Wireless Sensor Networks (UWSN) during the last years, still many challenges need to be successfully tackled before large-scale deployment of underwater sensor networks becomes a reality. UWSNs usually employ acoustic channels for communications, which compared with radio-frequency channels, allow much lower bandwidths and have longer propagation delays. In the past, different methods have been proposed to define how a node must acquire the channel in order to start a transmission. Given the large propagation delays of underwater communication channels, a TDMA-based approach may need big time-guards. On the other hand, the very same large propagation delay increases the occurrence of the hidden terminal problem in a CSMA-based approach. In this paper, impacts of utilization of different scheduling and retransmission techniques on an underwater routing protocol will be analyzed. This analysis, in which energy consumption, packet delay, number of duplicate packets, and packet loss are considered, will be carried out by means of simulation using the Network Simulator 3 and a subset of EDETA (Energyefficient aDaptive hiErarchical and robusT Architecture) routing protocol recently adapted to UWSN.
\end{abstract}

- S. Climent, A. Sanchez, J. V. Capella et al., 'Study of MAC Protocols for a Real Underwater Sensor Network Application', in The 2012 International Conference on Wireless Networks, 2012

Abstract Simulations have proven to be useful in aiding the research in wireless and underwater sensor networks. Simulations can be very convenient also when planning the development and deployment of an underwater sensor network for a real application. However, in order to achieve trustworthy results and to be able to extract correct conclusions, accurate models and real parameters should be used. This paper studies the behaviour of different medium access techniques when they are applied to a real monitoring application. To this end, different MACs where implemented and tested by means of simulation, using accurate models fed with real data.

- S. Climent, A. Sanchez, J. V. Capella et al., 'Simulating MAC protocols under real underwater sensor networks assumptions', in Proceedings of the Seventh ACM International Conference on Underwater Networks and Systems, ACM, New York, New York, USA: ACM Press, Nov. 2012, p. 34, ISBN: 9781450317733 
Abstract Underwater Wireless Sensor Networks (UWSN) have become an important area of research due to its many possible applications. One example are the long-term monitoring applications were the nodes only need to be awake during a small fraction of time. This kind of applications can greatly benefit from low-power, wake-up systems. However, despite the fact that the simulations can greatly improve the development time of new algorithms and features, optimizing their performance, up until today there is no wake-up system model available. In this paper a low-power underwater wake-up model for the ns-3 simulator is going to be presented. Using this model the, as far as we know, only two available underwater modems with integrated wake-up capabilities are compared in terms of energy consumption.

- S. Climent, A. Sanchez, J. V. Capella et al., 'Simulating MAC protocols under real underwater sensor networks assumptions', in Proceedings of the Seventh ACM International Conference on Underwater Networks and Systems, ACM, New York, New York, USA: ACM Press, Nov. 2012, p. 34, ISBN: 9781450317733

Abstract Many research areas have benefited from simulations to acquire reliable data before conducting real experiments. In order to obtain these data, the actual system has to be precisely modelled and fed with accurate parameters. Sensor networks can and also have taken advantage from simulations. For example, they can be very convenient when planning the development and deployment of an underwater sensor network. This paper presents the behaviour of different medium access techniques by means of simulation and employing accurate models fed with real data. Parameters like energy consumption and packet delay are measured and the impact of the transmission speed on the performance of the protocols is studied. 


\section{Appendix A}

\section{Simulation Results of the MAC Study}

This appendix includes all the results regarding the MAC study that were not included in Chapter 5 for brevity. Nevertheless, all the important trends and conclusions are shown in the aforementioned chapter and this appendix is included as a reference for the interested reader. 


\section{A.1 Thoughput vs backoff study}

This section includes all the results regarding the throughput vs backoff study. As explained in Chapter 5, T-Lohi was left from this study since its backoff time is given by the protocol specification and dependent on the maximum propagation time.
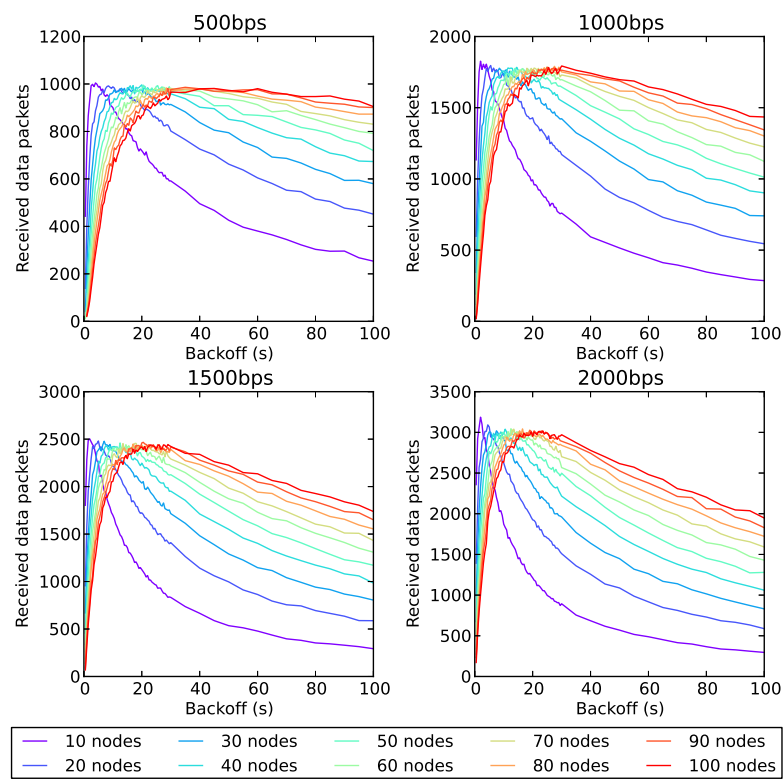

Figure A.1: Aloha throughput vs backoff analysis 

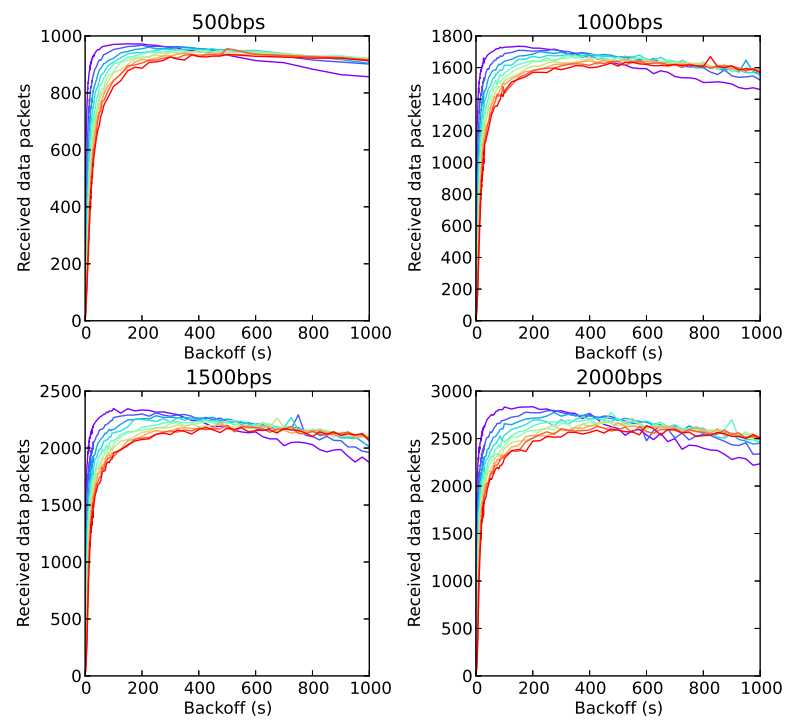

- 10 nodes -30 nodes -50 nodes

Figure A.2: Aloha with ACK throughput vs backoff analysis
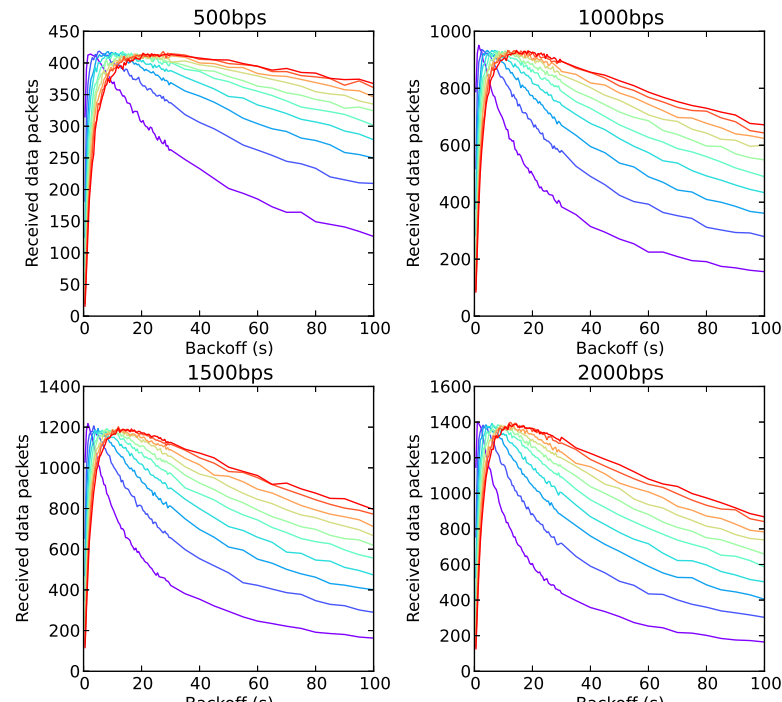

-10 nodes -30 nodes -50 nodes
-20 nodes -40 nodes -60 node

50 nodes -70 nodes -90 nodes
60 nodes -80 nodes -100 nodes

Figure A.3: FAMA throughput vs backoff analysis 

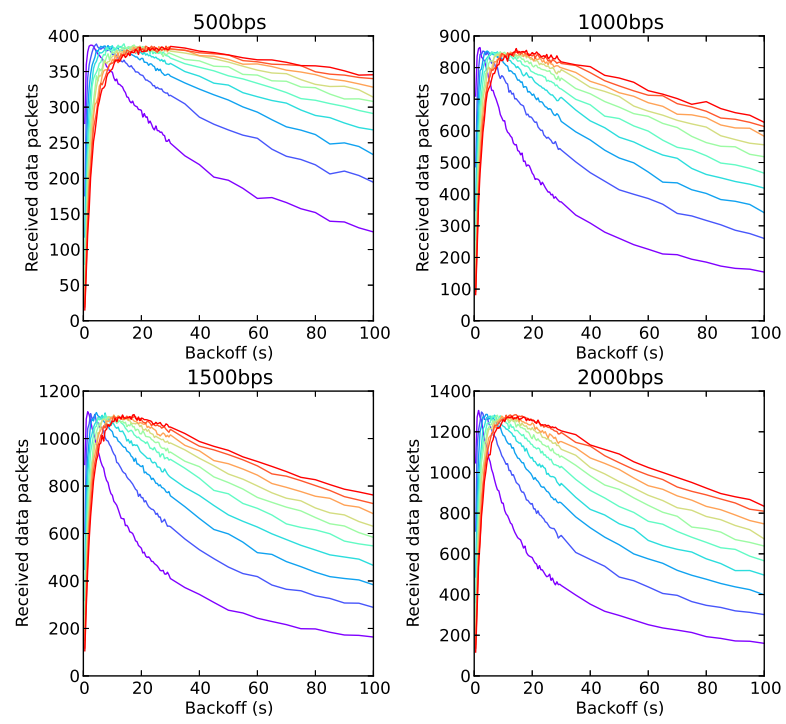

- 10 nodes -30 nodes -50 nodes

- 70 nodes -90 nodes

Figure A.4: FAMA with ACK throughput vs backoff analysis
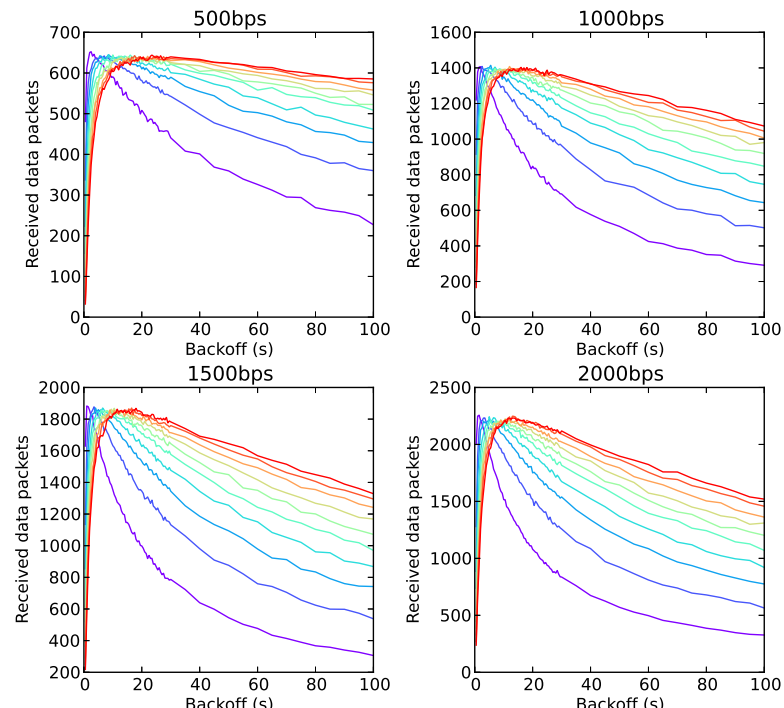

-10 nodes -30 nodes -50 nodes
-20 nodes -40 nodes

70 nodes -90 nodes
-80 nodes -100 nodes

Figure A.5: FAMA X2 throughput vs backoff analysis 

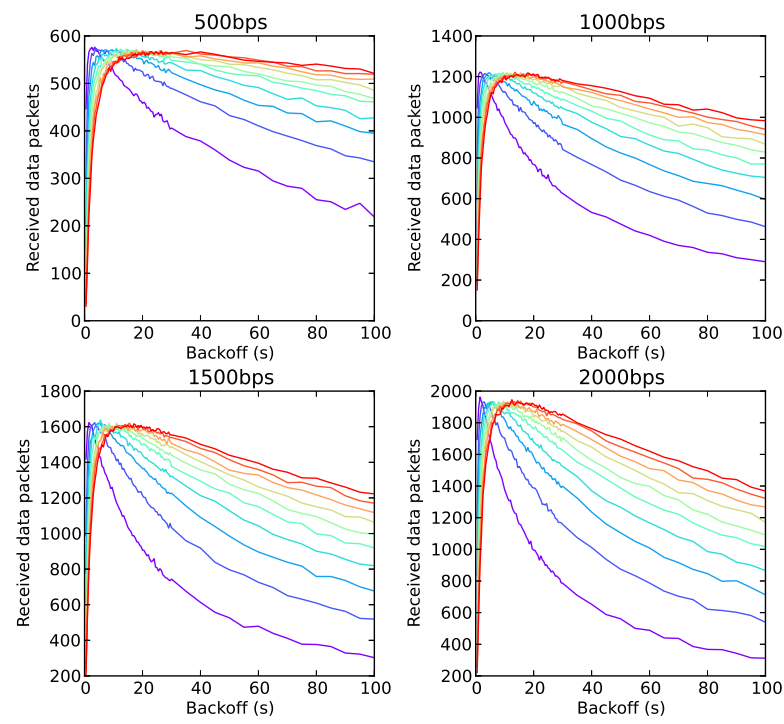

- 10 nodes -30 nodes -50 nodes

70 nodes -90 nodes

Figure A.6: FAMA X2 with ACK throughput vs backoff analysis
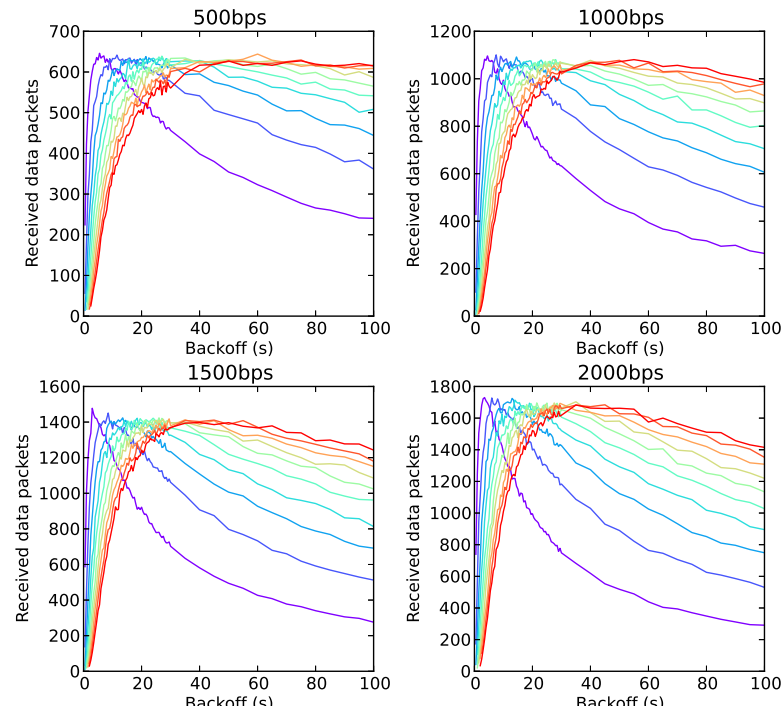

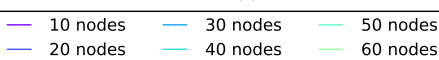

70 nodes -90 nodes
-80 nodes -100 nodes

Figure A.7: MACA throughput vs backoff analysis 

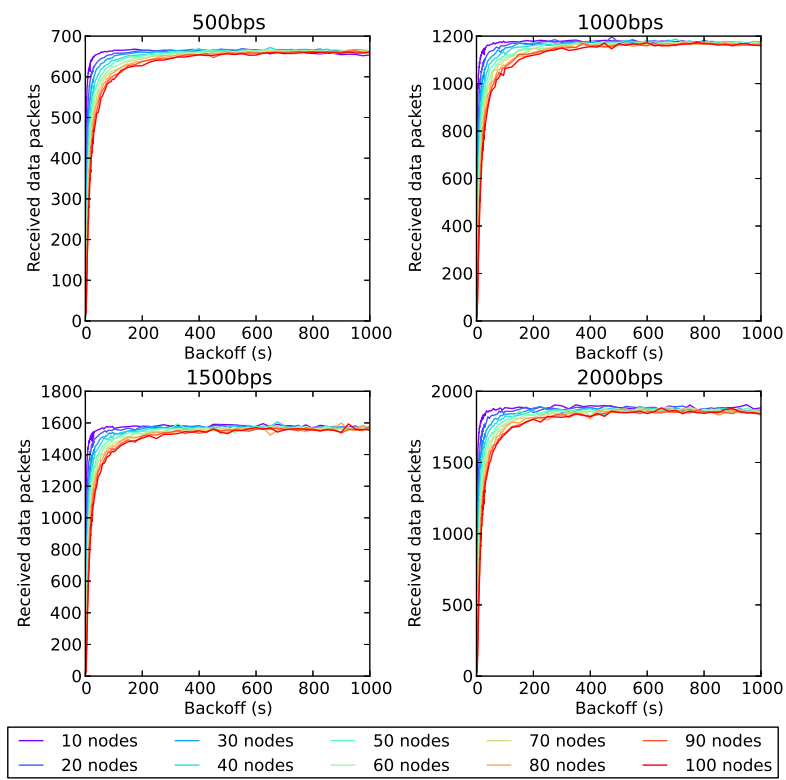

Figure A.8: MACA with ACK throughput vs backoff analysis 


\section{A.2 Delay vs backoff study}

This section includes all the results regarding the delay vs backoff study. As explained in Chapter 5, T-Lohi was left from this study since its backoff time is given by the protocol specification and dependent on the maximum propagation time.
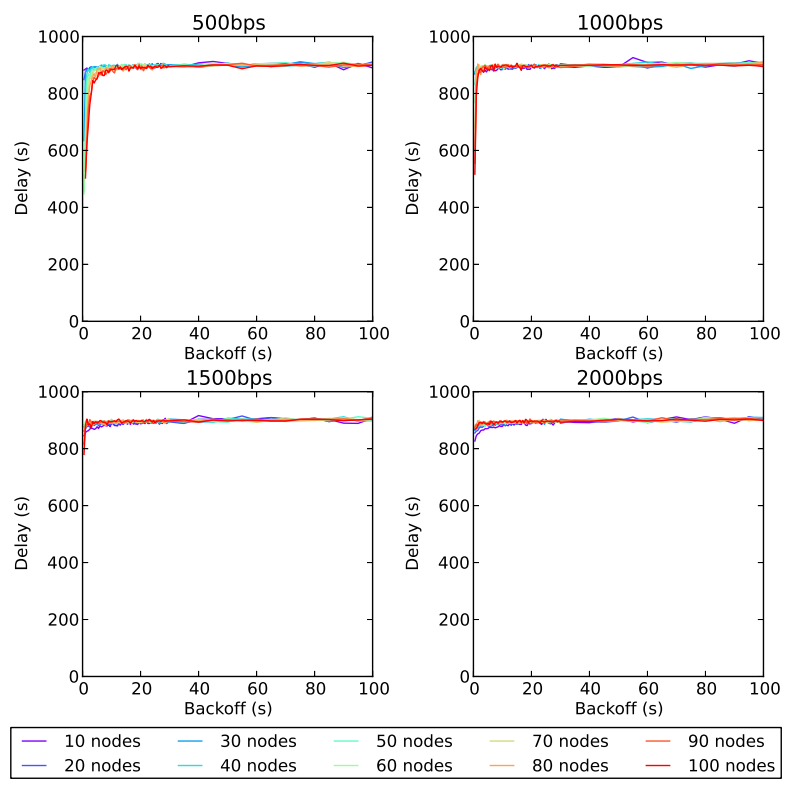

Figure A.9: Aloha delay vs backoff analysis 

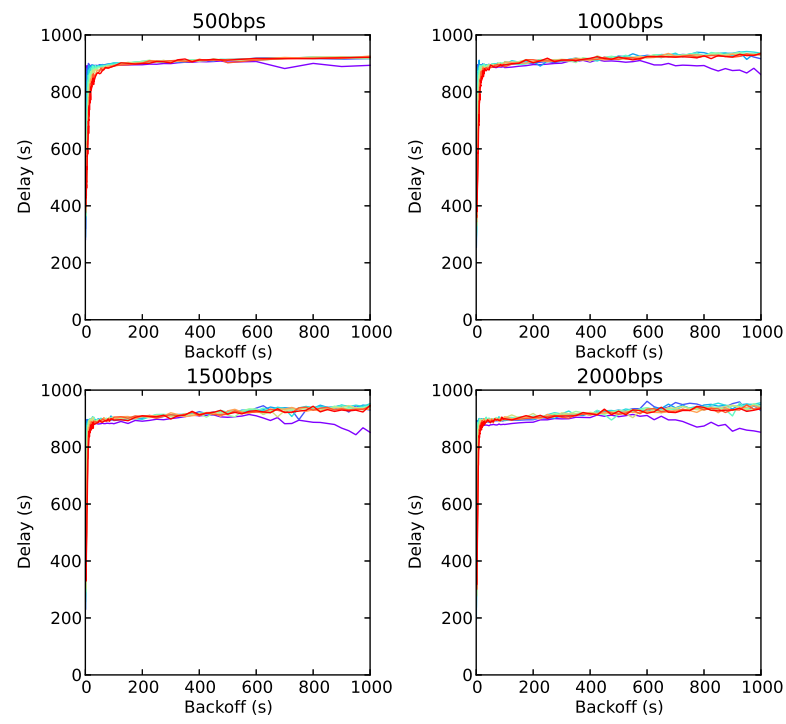

- 10 nodes -30 nodes -50 nodes

60 nodes -70 nodes -90 nodes

Figure A.10: Aloha with ACK delay vs backoff analysis
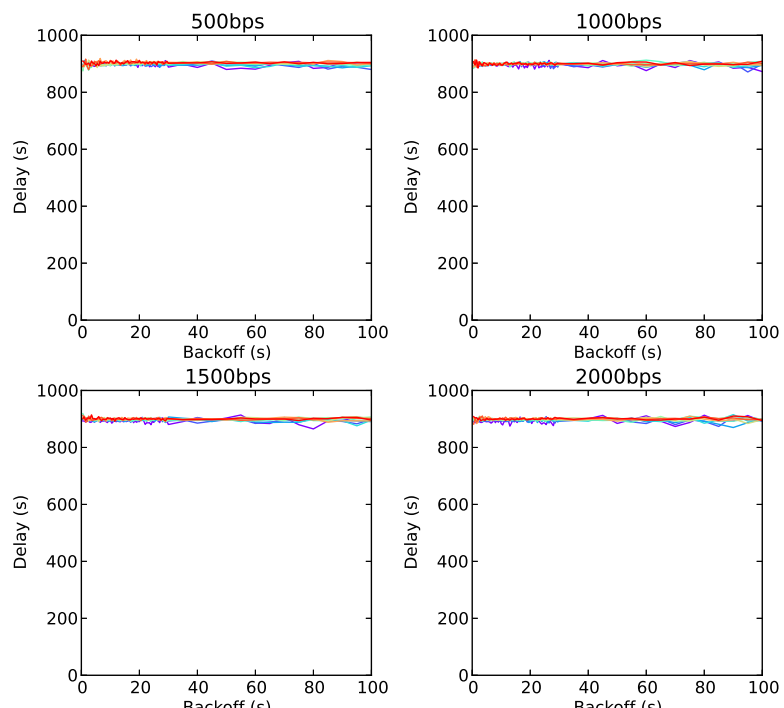

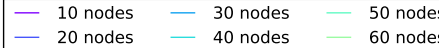

70 nodes -90 nodes

Figure A.11: FAMA delay vs backoff analysis 

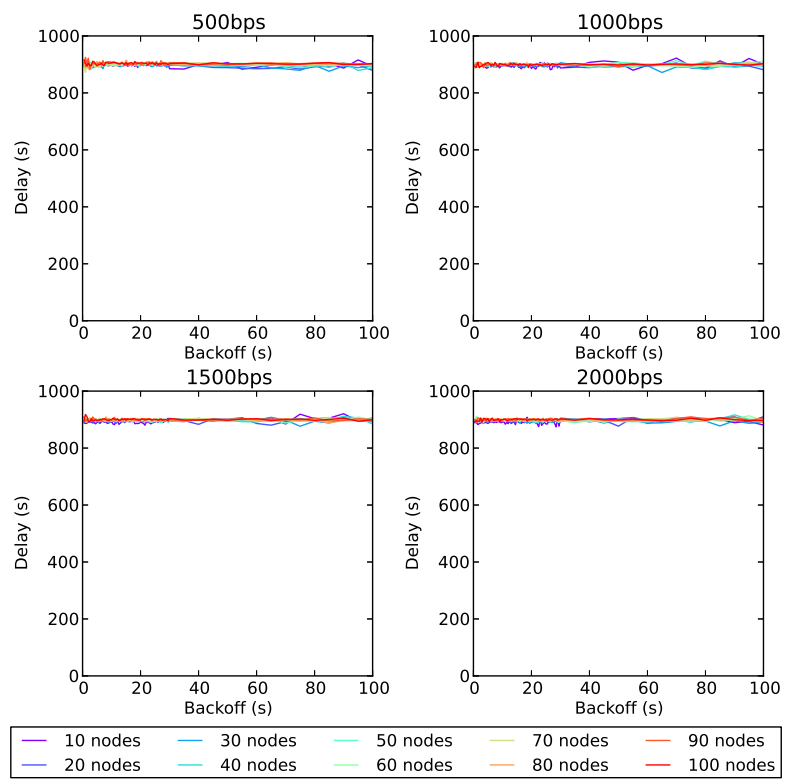

Figure A.12: FAMA with ACK delay vs backoff analysis
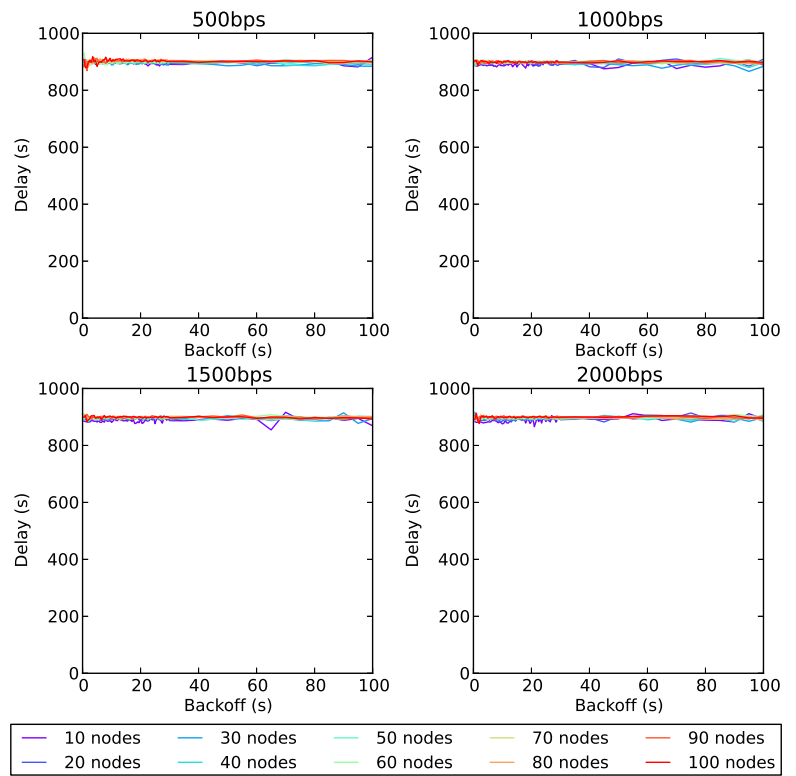

Figure A.13: FAMA X2 delay vs backoff analysis 

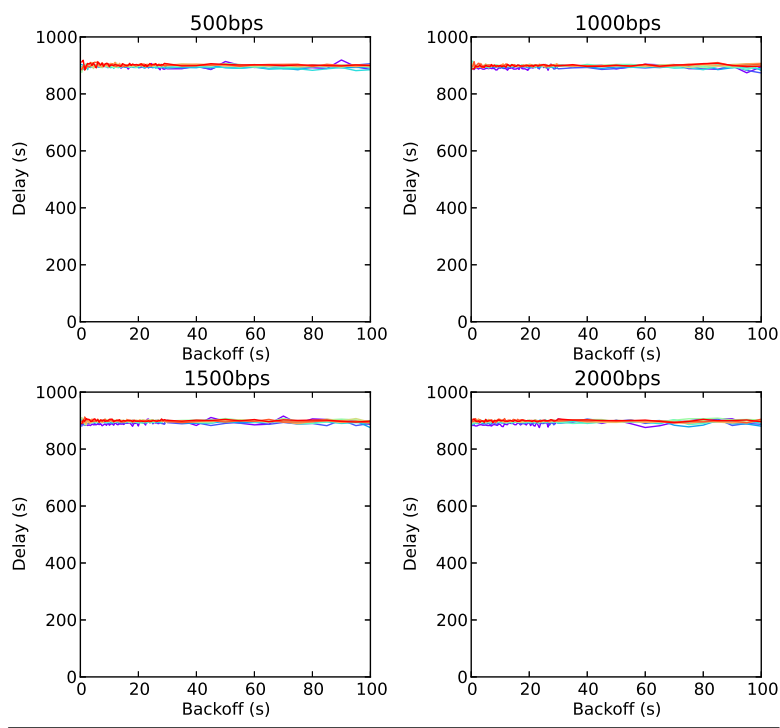

- 10 nodes -30 nodes -50 nodes

Figure A.14: FAMA X2 with ACK delay vs backoff analysis
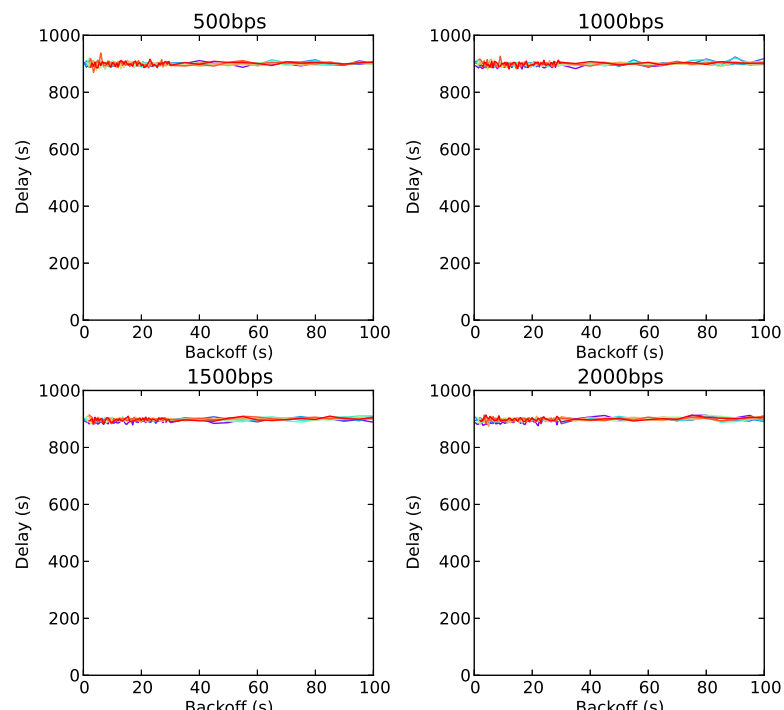

-10 nodes -30 nodes -50 nodes
-20 nodes -40 nodes

70 nodes -90 nodes

nodes -80 nodes

Figure A.15: MACA delay vs backoff analysis 

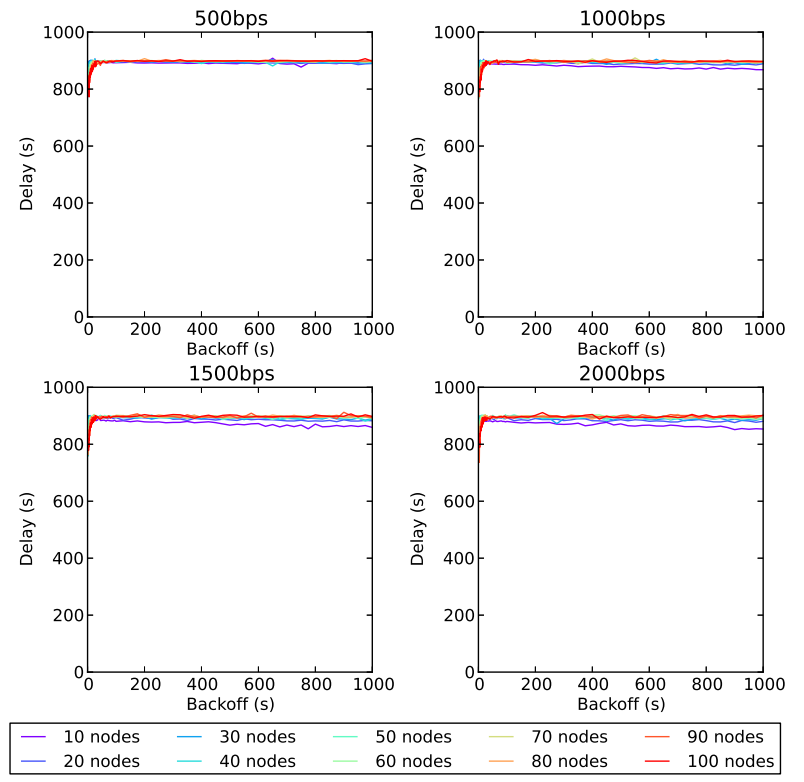

Figure A.16: MACA with ACK delay vs backoff analysis 


\section{A.3 Energy vs backoff study}

This section includes all the results regarding the energy vs backoff study. As explained in Chapter 5, T-Lohi was left from this study since its backoff time is given by the protocol specification and dependent on the maximum propagation time.
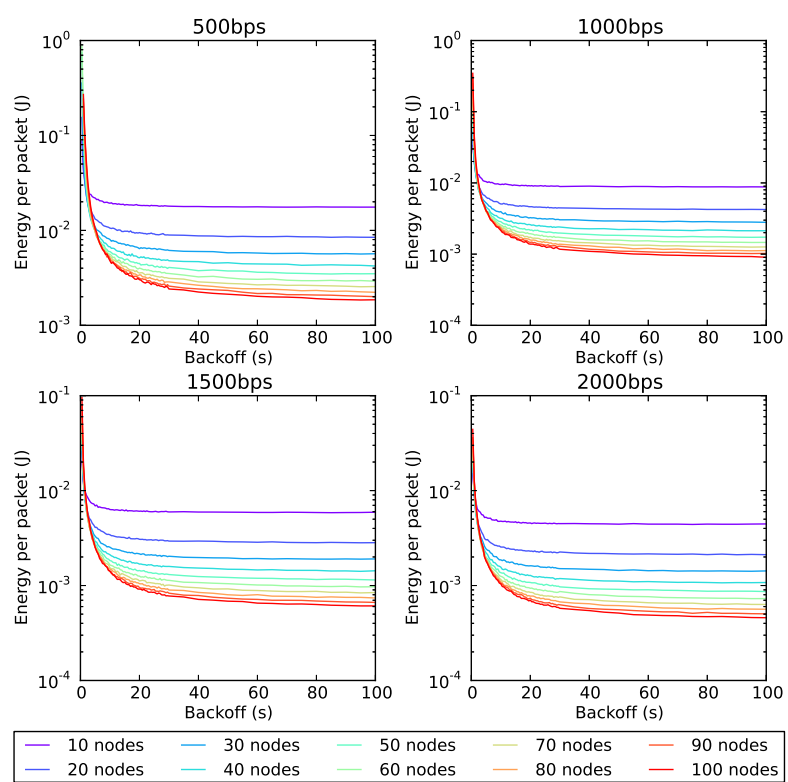

Figure A.17: Aloha energy vs backoff analysis 

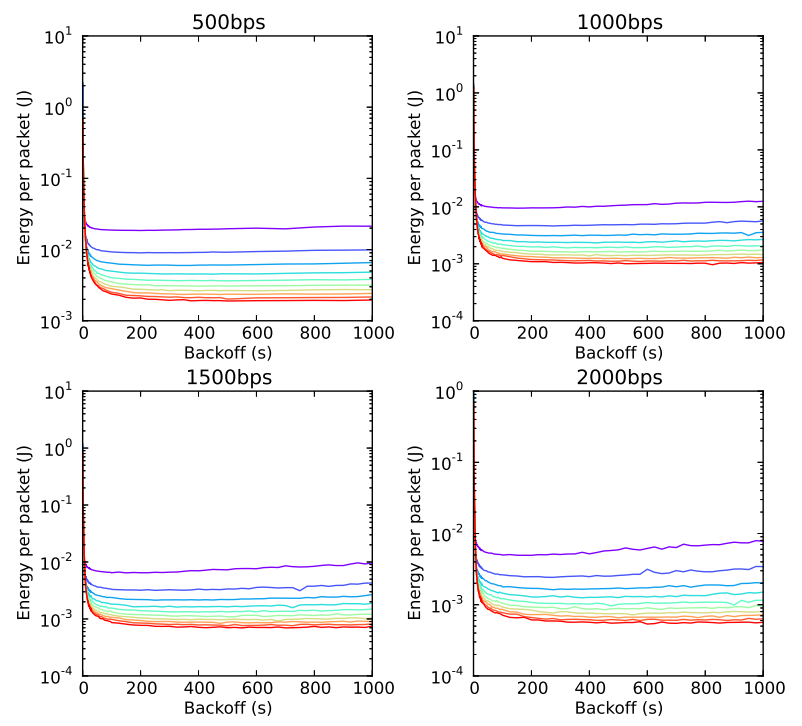

- 10 nodes -30 nodes -50 nodes

70 nodes -90 nodes

20 nodes

nodes

Figure A.18: Aloha with ACK energy vs backoff analysis
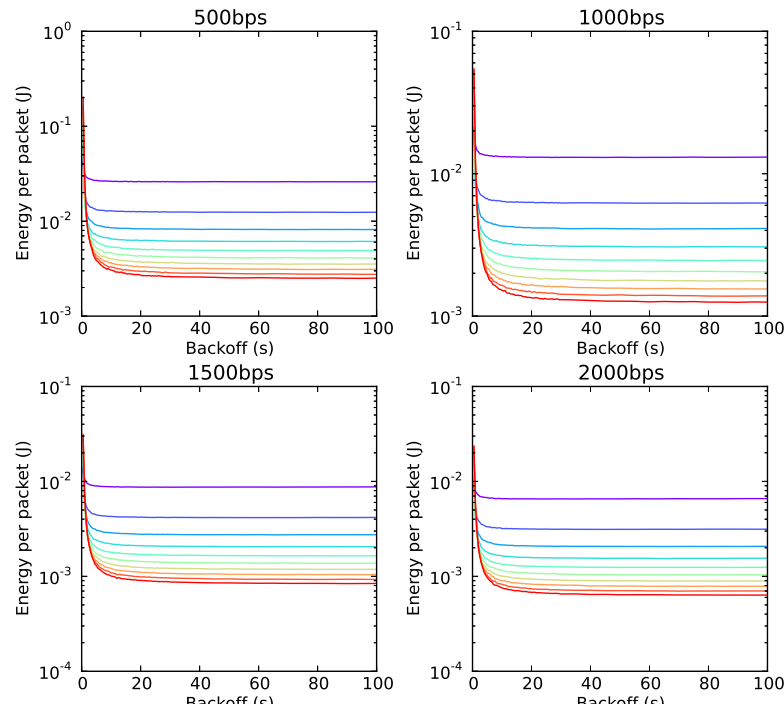

\begin{tabular}{|c|c|c|c|c|}
\hline - 10 nodes & - 30 nodes & $\begin{array}{l}50 \text { nodes } \\
60 \text { nodes }\end{array}$ & $\begin{array}{l}70 \text { nodes } \\
80 \text { nodes }\end{array}$ & $\begin{array}{l}\text { _ } 90 \text { nodes } \\
\text { _ } 100 \text { nodes }\end{array}$ \\
\hline
\end{tabular}

Figure A.19: FAMA energy vs backoff analysis 

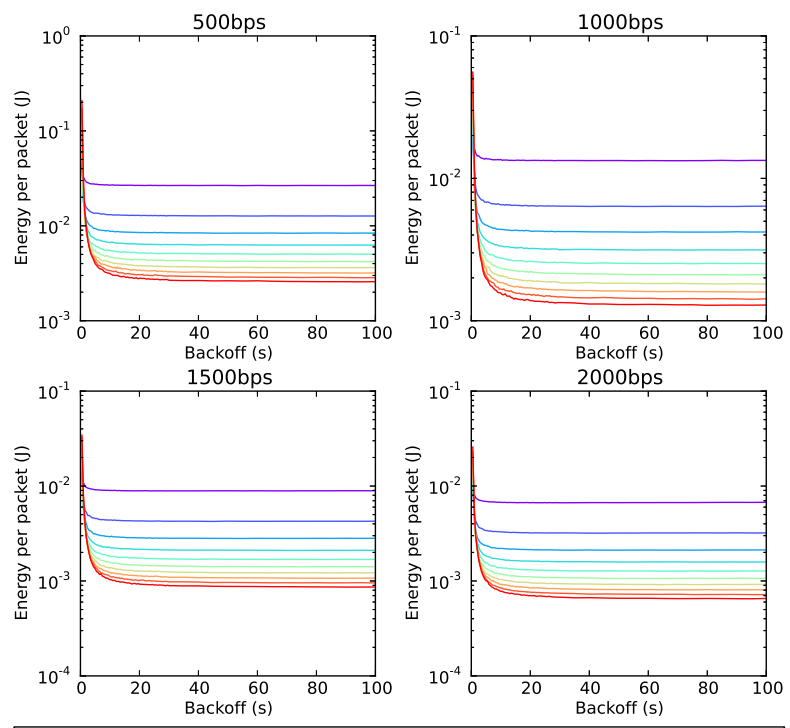

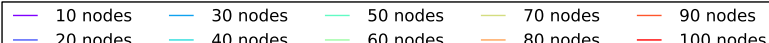

Figure A.20: FAMA with ACK energy vs backoff analysis
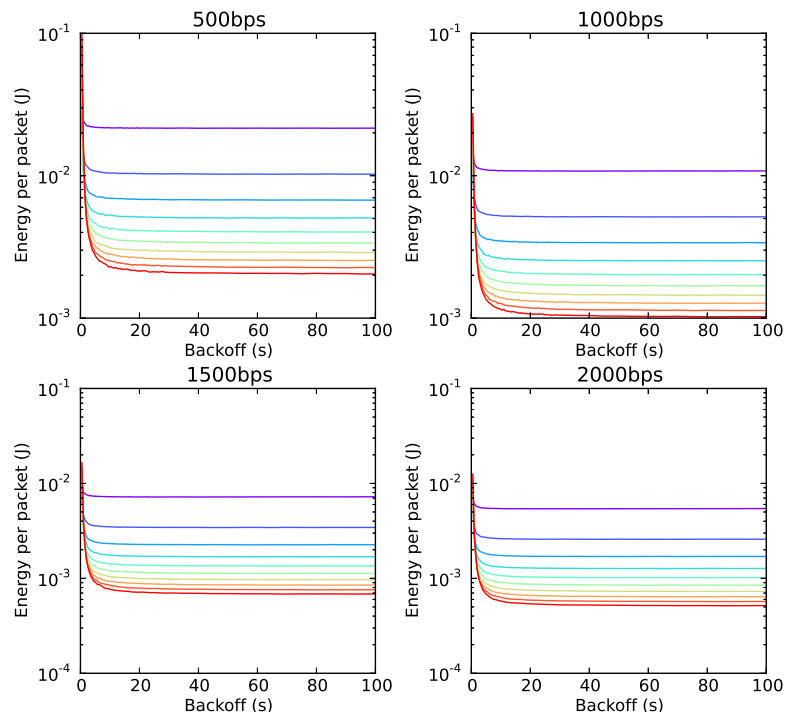

- 10 nodes -30 nodes -50 nodes 50 nodes -70 nodes -90 nodes 30 nodes
40 nodes

Figure A.21: FAMA X2 energy vs backoff analysis 

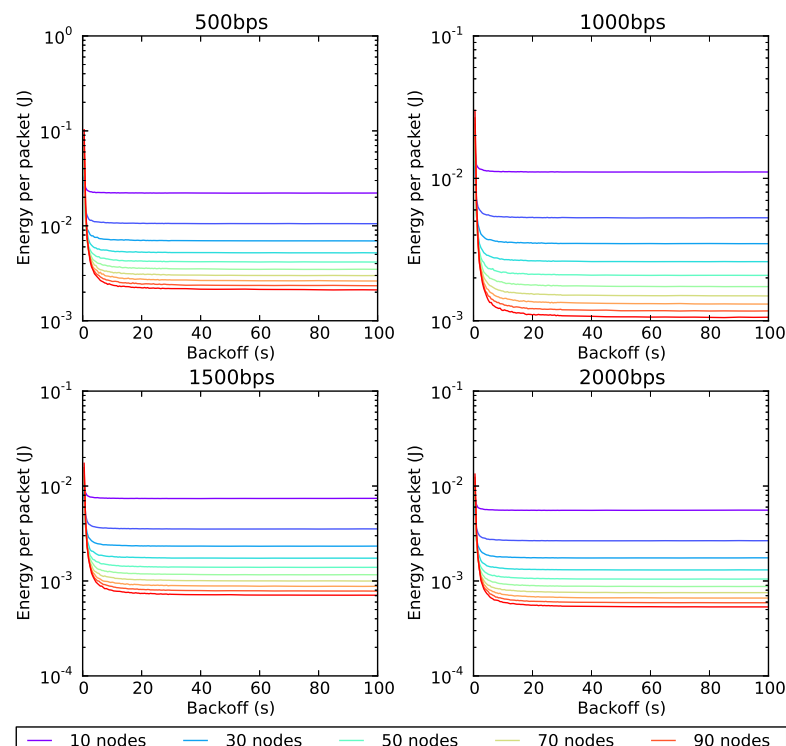

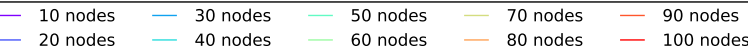

Figure A.22: FAMA X2 with ACK energy vs backoff analysis
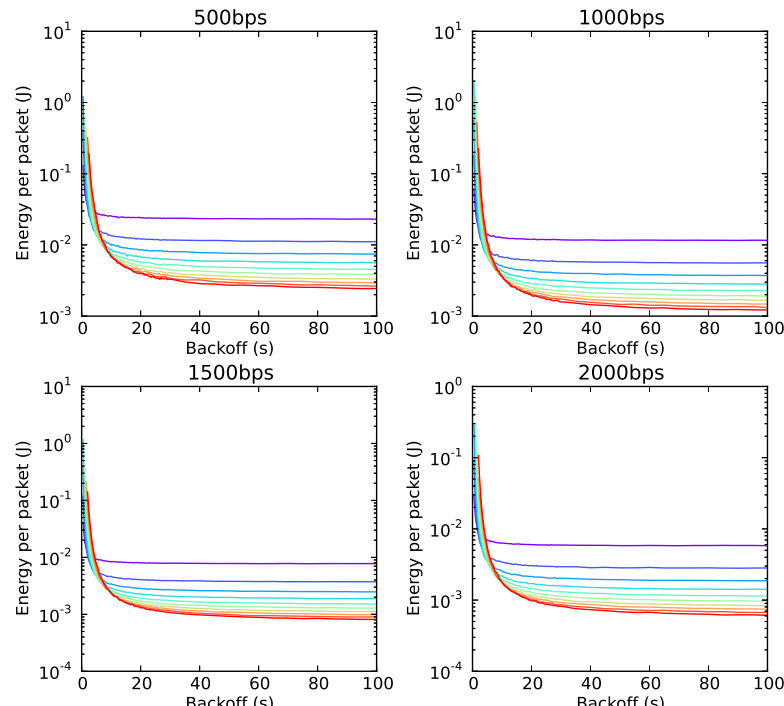

\begin{tabular}{|lrrrr|}
\hline 10 nodes & -30 nodes & 50 nodes & -70 nodes & -90 nodes \\
-20 nodes & -40 nodes & 60 nodes & -80 nodes & -100 nodes
\end{tabular}

Figure A.23: MACA energy vs backoff analysis 

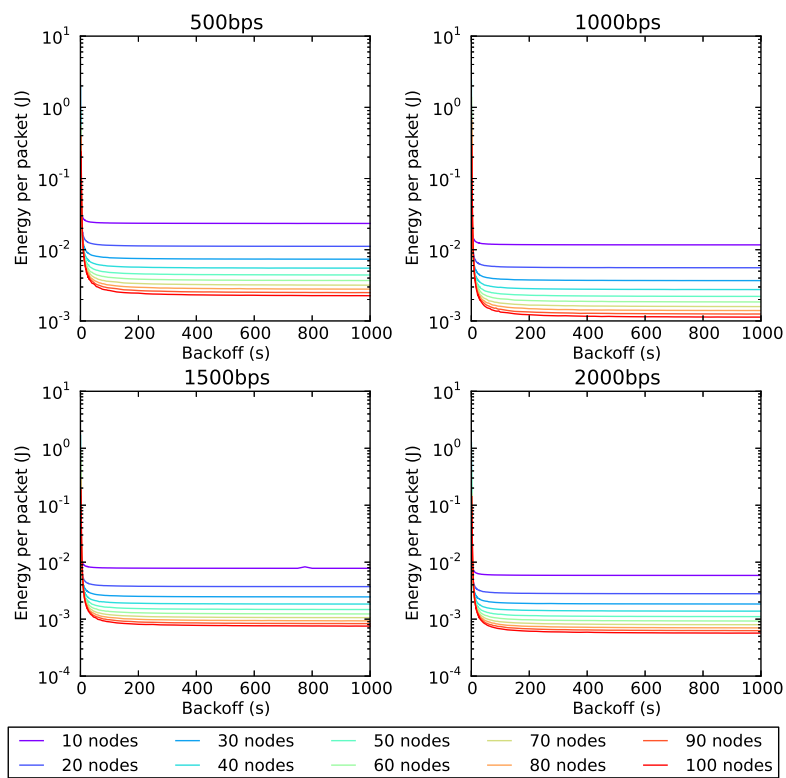

Figure A.24: MACA with ACK energy vs backoff analysis 


\section{A.4 Sink energy vs backoff study}

This section includes all the results regarding the sink energy vs backoff study. As explained in Chapter 5, T-Lohi was left from this study since its backoff time is given by the protocol specification and dependent on the maximum propagation time.
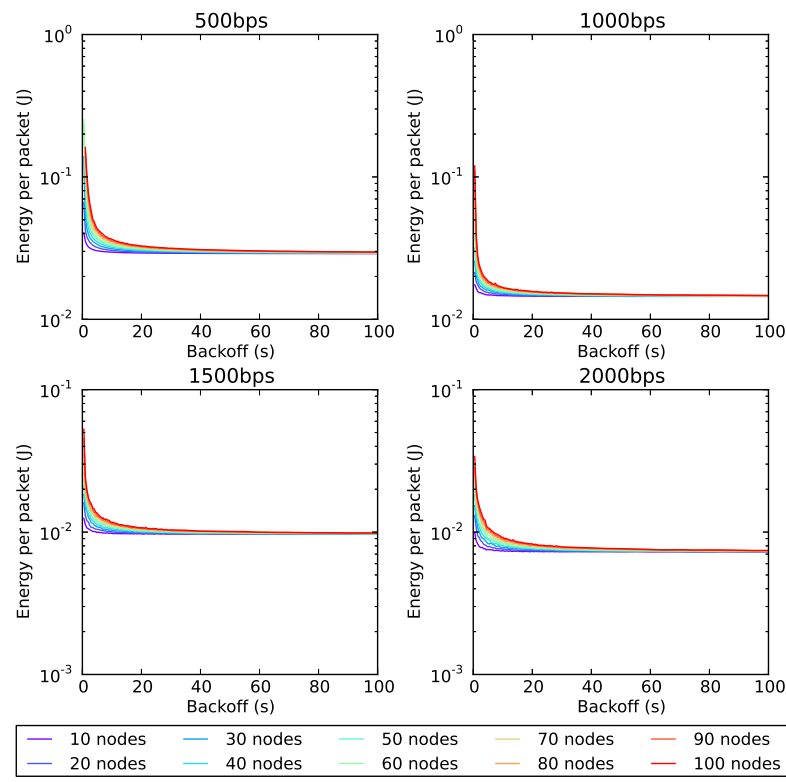

Figure A.25: Aloha sink energy vs backoff analysis 

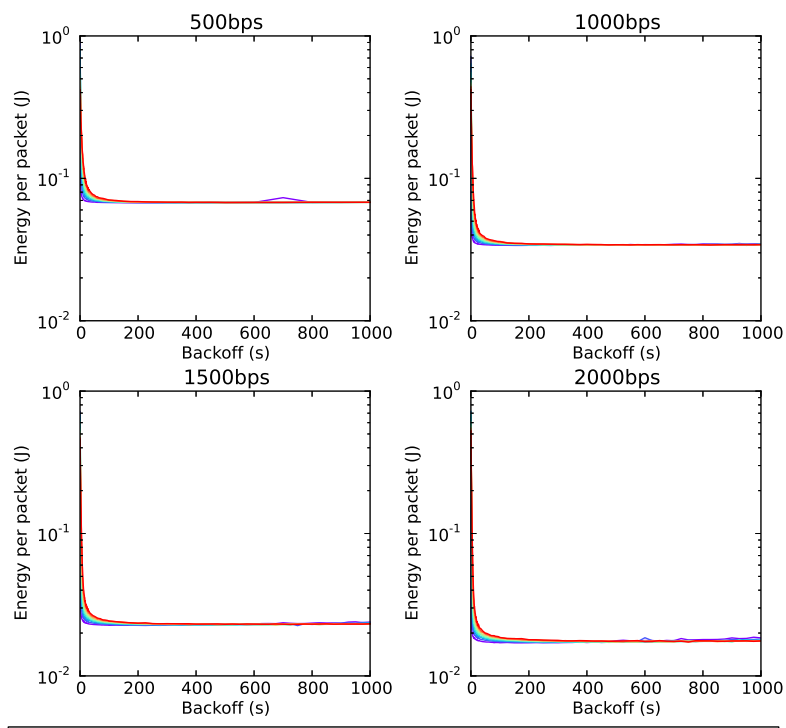

- 10 nodes -30 nodes

50 nodes -70 nodes -90 nodes
60 nodes

Figure A.26: Aloha with ACK sink energy vs backoff analysis
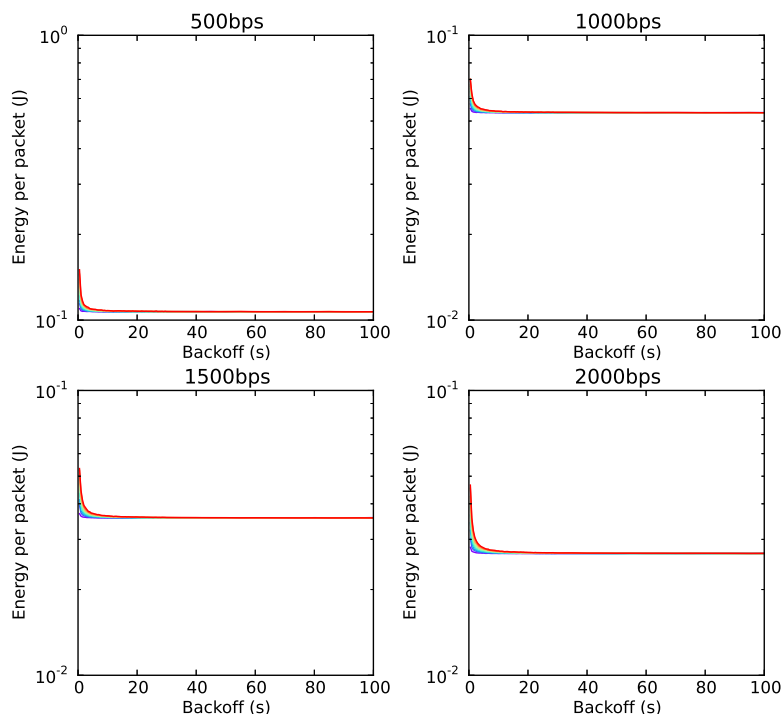

-10 nodes -30 nodes -50 nodes
-20 nodes -40 nodes

70 nodes 90 nodes

Figure A.27: FAMA sink energy vs backoff analysis 

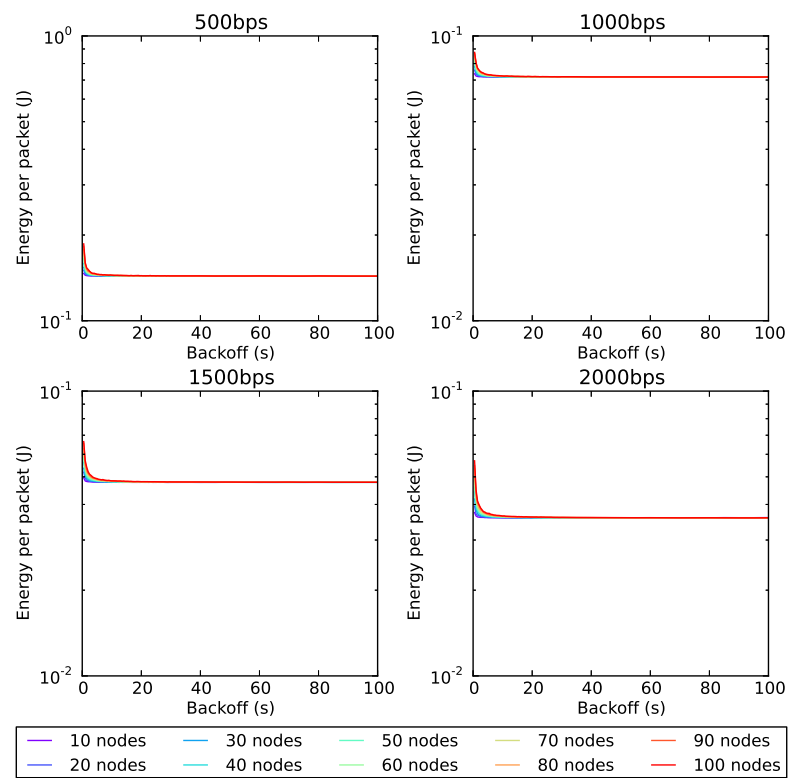

Figure A.28: FAMA with ACK sink energy vs backoff analysis
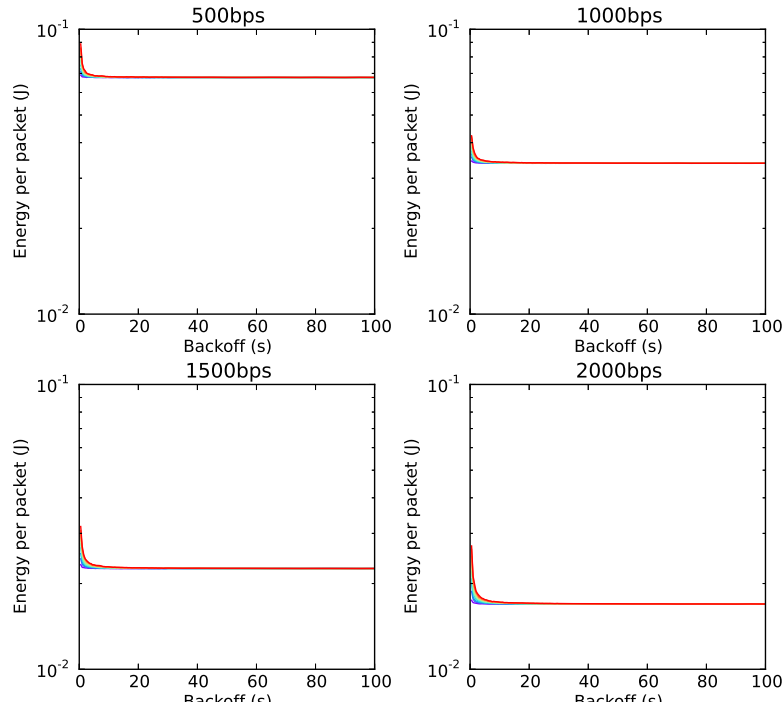

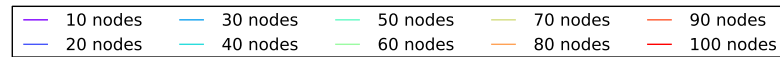

Figure A.29: FAMA X2 sink energy vs backoff analysis 

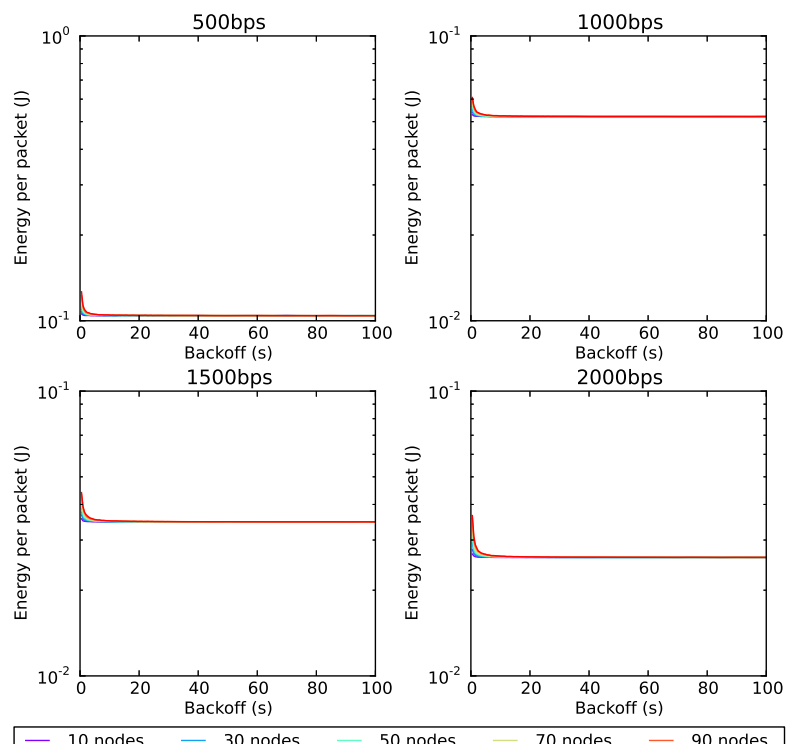

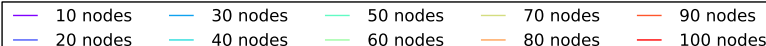

Figure A.30: FAMA X2 with ACK sink energy vs backoff analysis
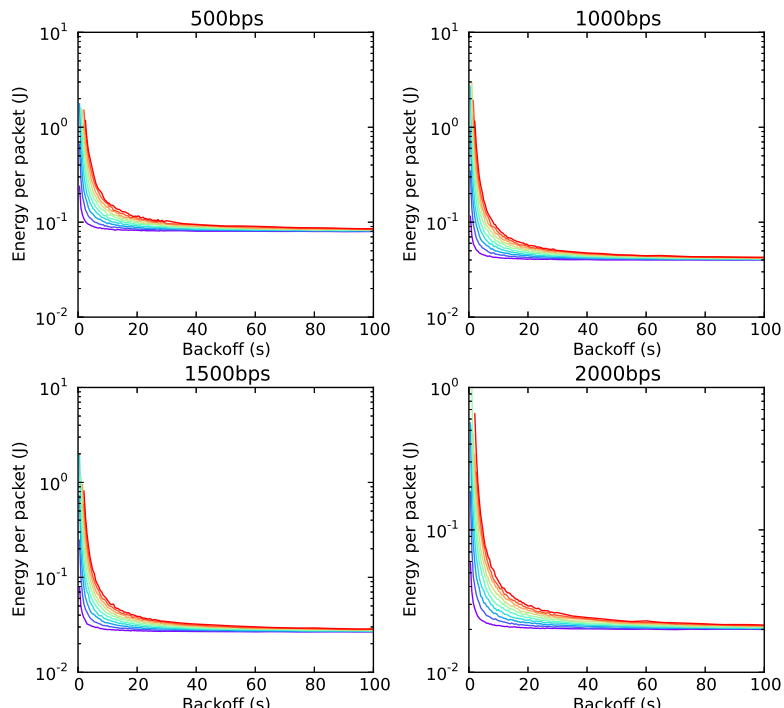

-10 nodes -30 nodes -50 nodes
-20 nodes -40 nodes

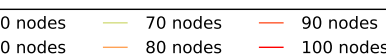

nodes -80 nodes -100 nodes

Figure A.31: MACA sink energy vs backoff analysis 

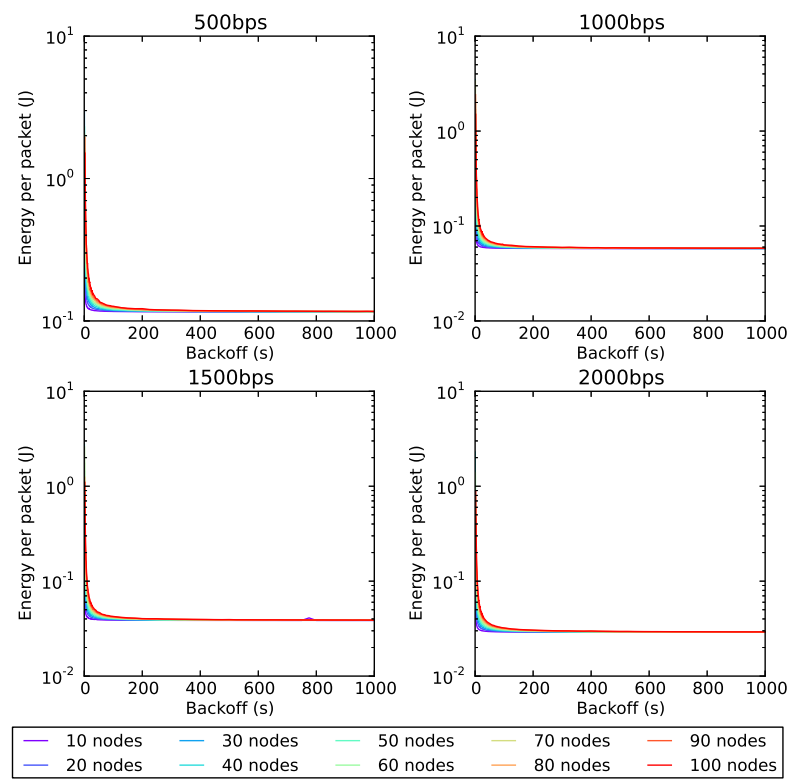

Figure A.32: MACA with ACK sink energy vs backoff analysis 


\section{A.5 Estimation of the optimum backoff}

This section includes all the results regarding the estimation of the optimum backoff. As explained in Chapter 5, T-Lohi was left from this study since its backoff time is given by the protocol specification and dependent on the maximum propagation time.
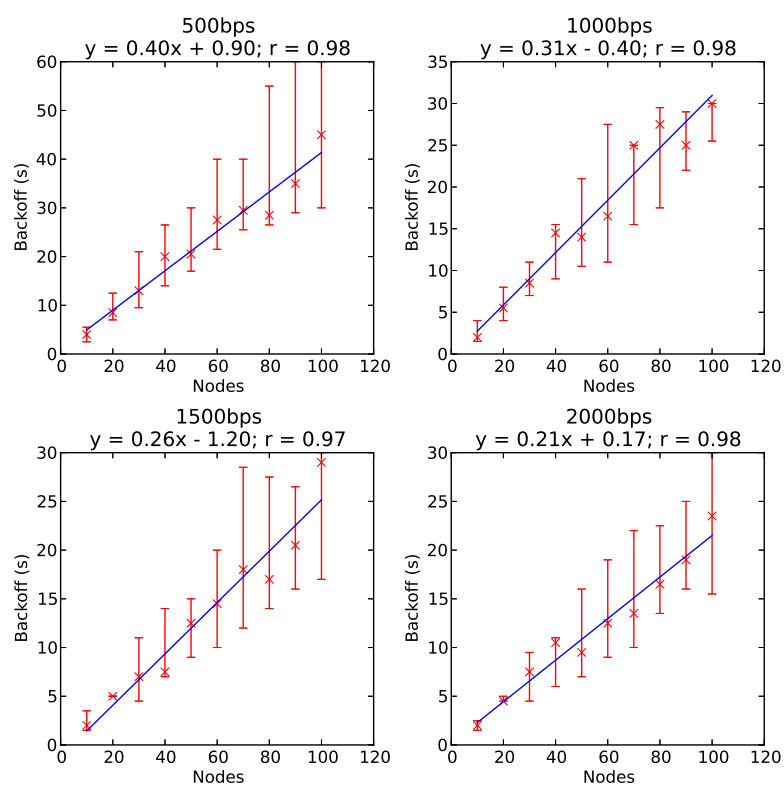

Figure A.33: Aloha optimum backoff analysis 

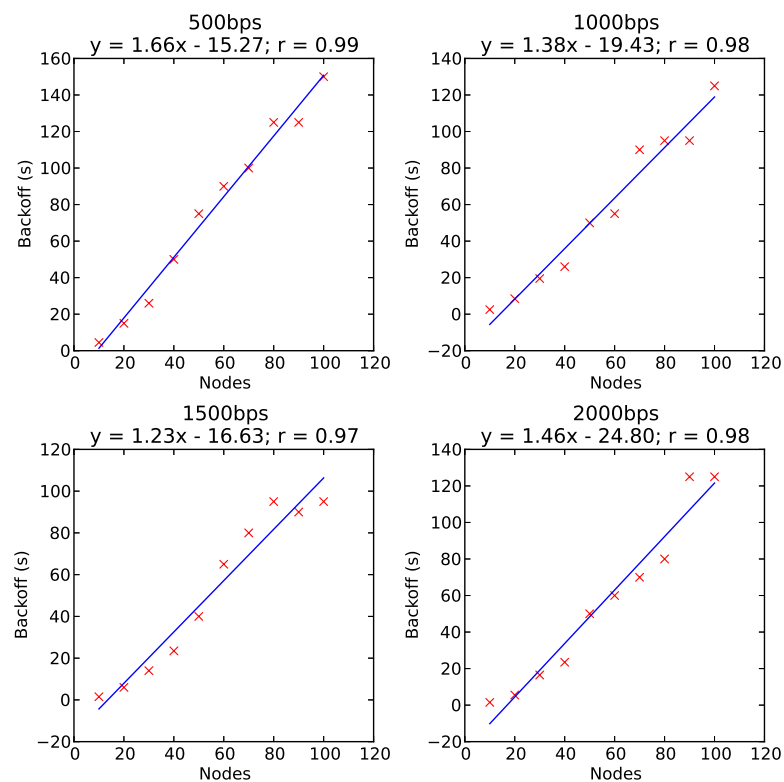

Figure A.34: Aloha with ACK optimum backoff analysis
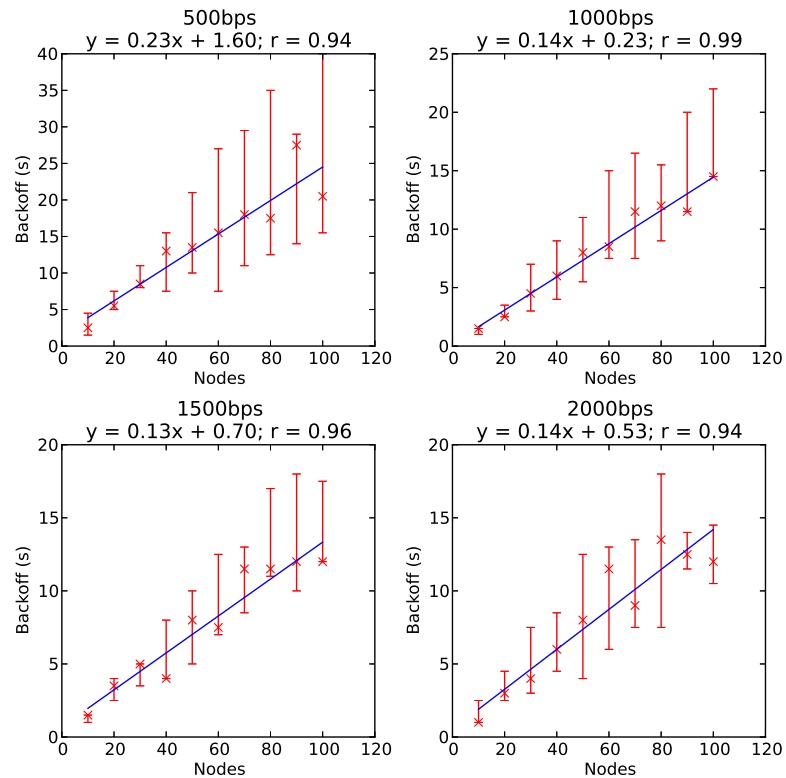

Figure A.35: FAMA optimum backoff analysis 

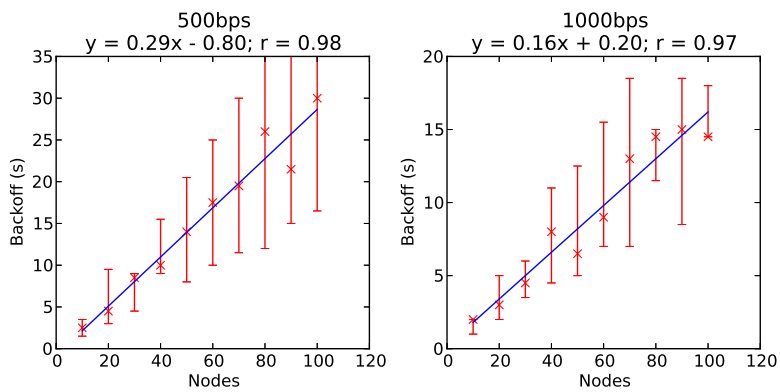

$1500 \mathrm{bps}$
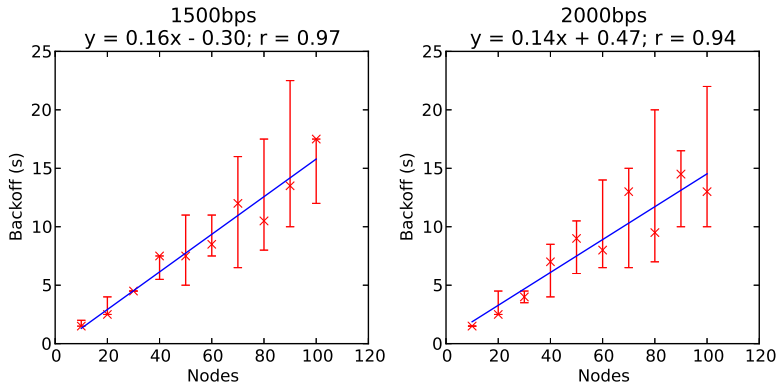

Figure A.36: FAMA with ACK optimum backoff analysis
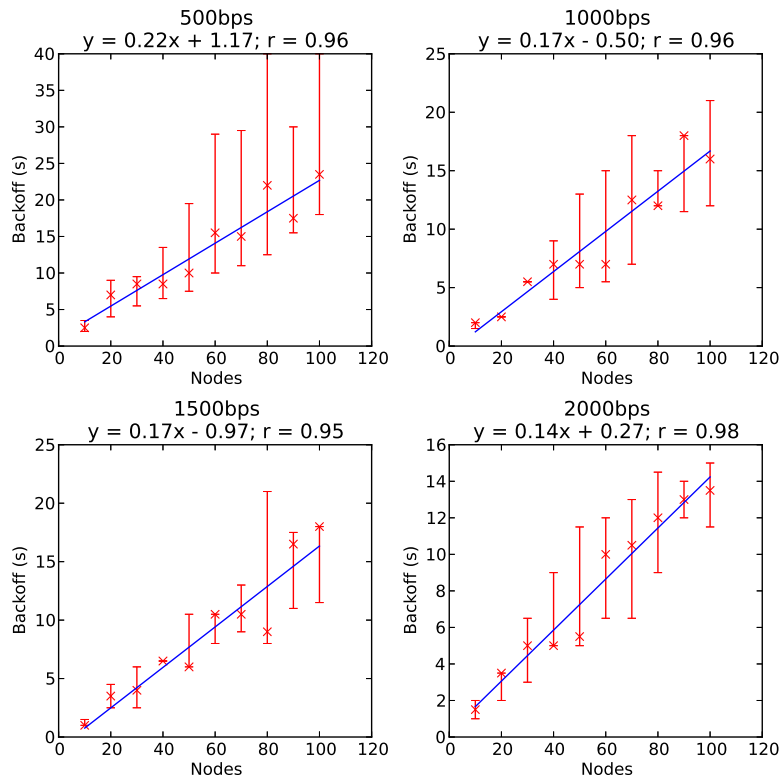

Figure A.37: FAMA X2 optimum backoff analysis 

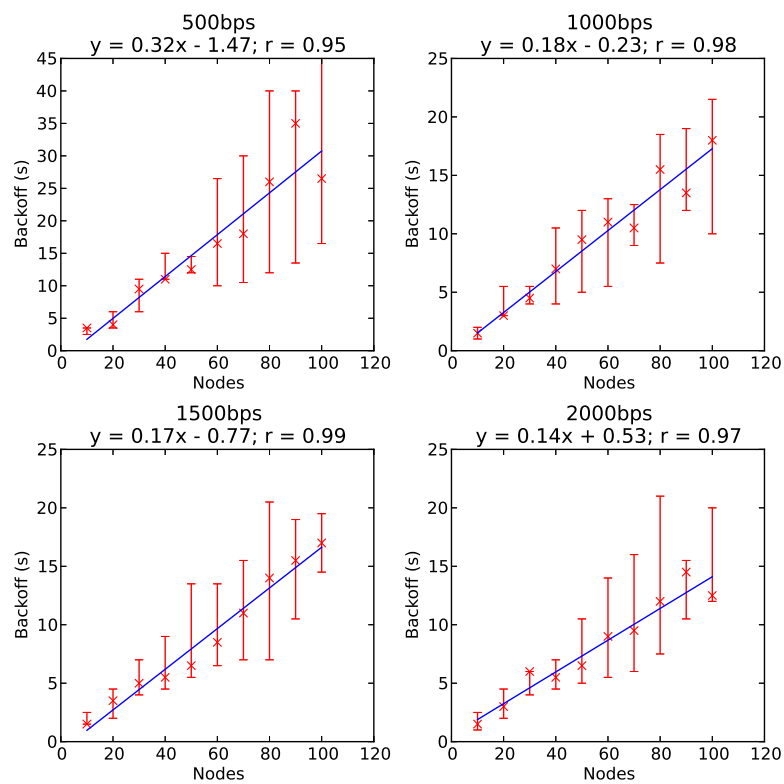

Figure A.38: FAMA X2 with ACK optimum backoff analysis
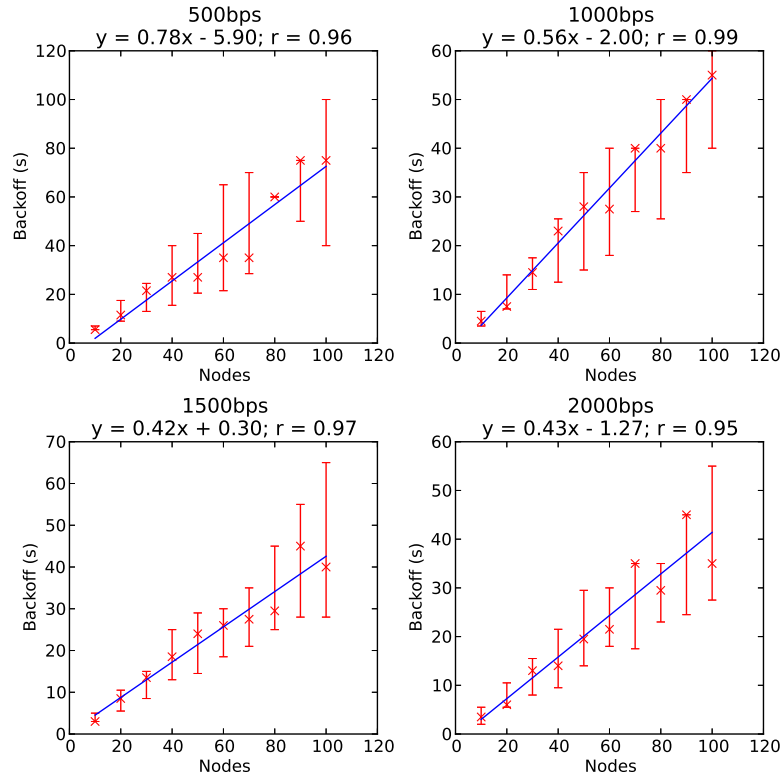

Figure A.39: MACA optimum backoff analysis 

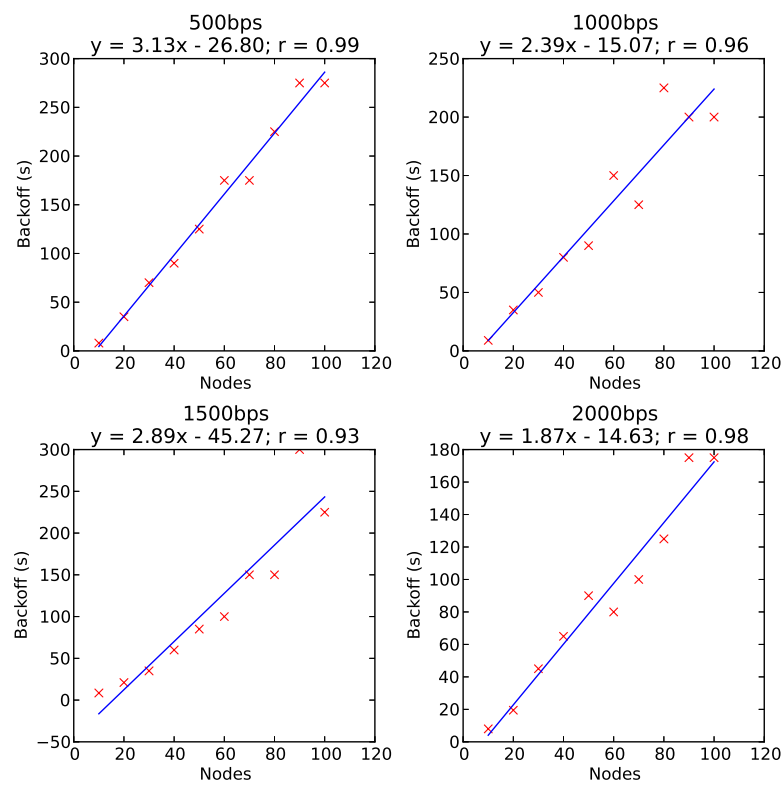

Figure A.40: MACA with ACK optimum backoff analysis 


\section{A.6 Influence of acknowledgements on throughput}

This section includes all the results regarding the influence of acknowledgements on throughput. As explained in Chapter 5, all protocols use the backoff time that offers their better performance and T-Lohi uses the one given by the protocol specification and dependent on the maximum propagation time.
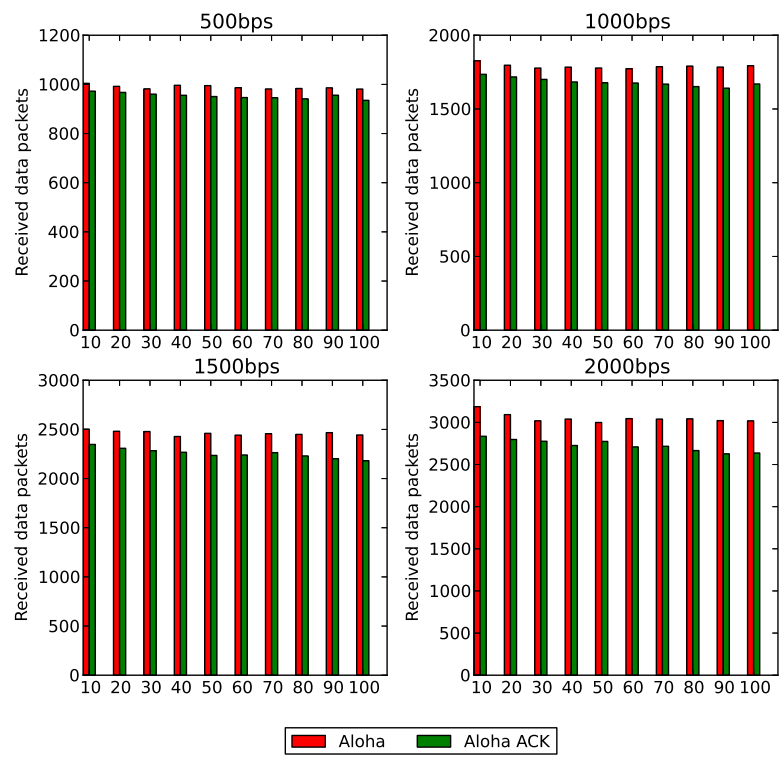

Figure A.41: Acknowledgements influence on throughput Aloha 

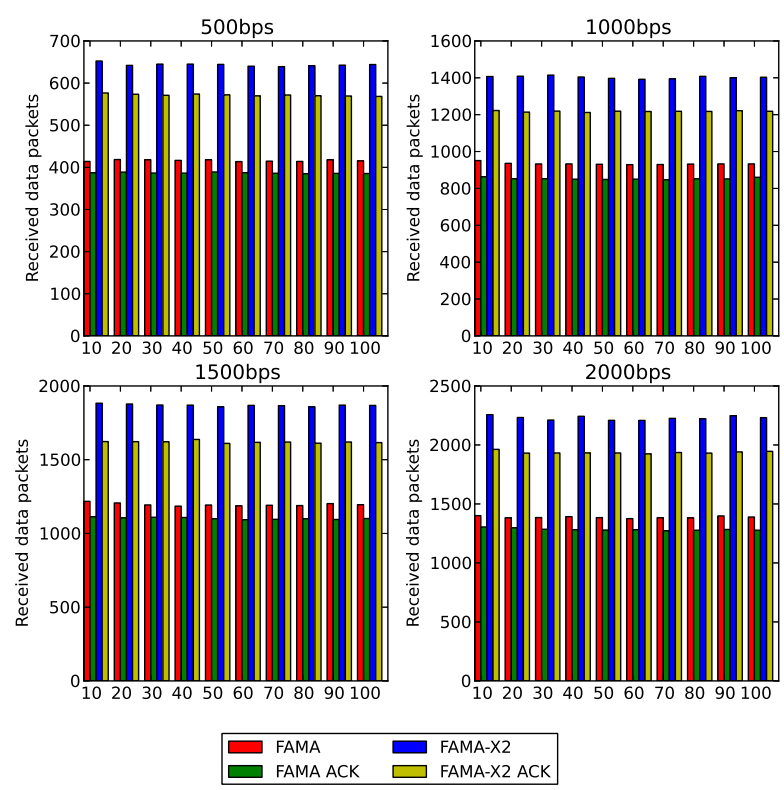

Figure A.42: Acknowledgements influence on throughput FAMA
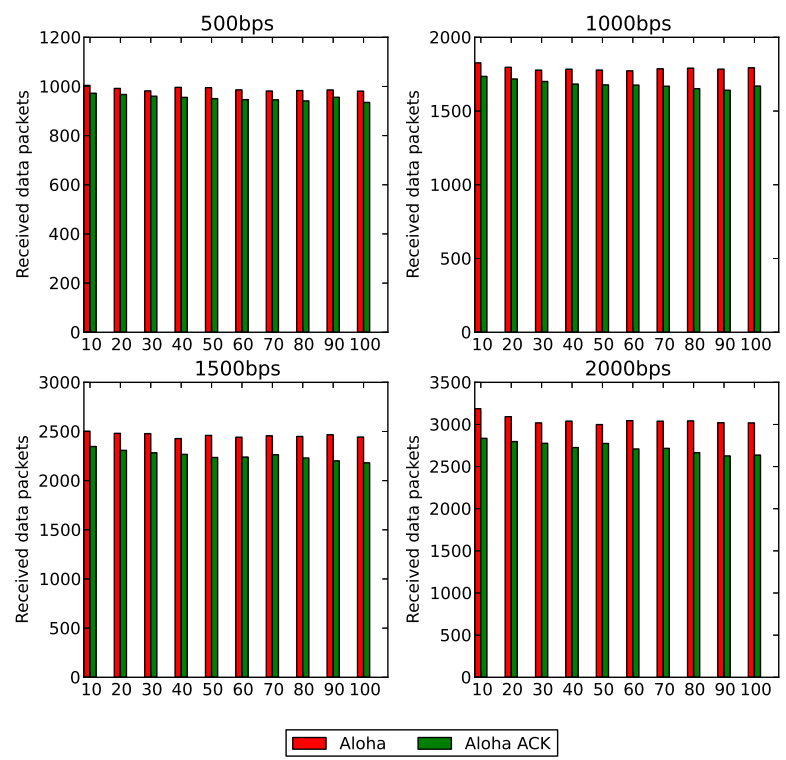

Figure A.43: Acknowledgements influence on throughput MACA 

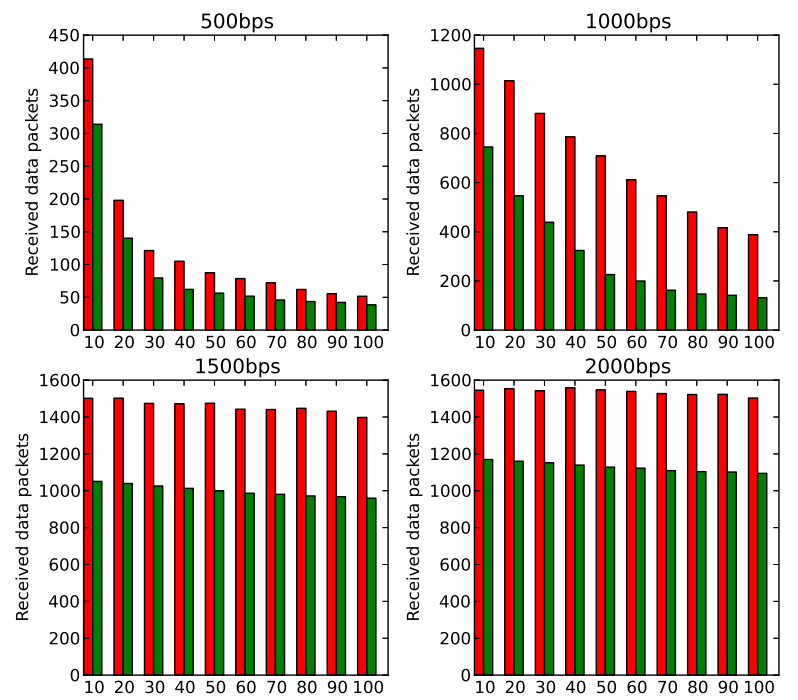

T-Lohi $\square$ T-Lohi-ACK

Figure A.44: Acknowledgements influence on throughput T-Lohi 


\section{A.7 Influence of acknowledgements on delay}

This section includes all the results regarding the influence of acknowledgements on delay. As explained in Chapter 5, all protocols use the backoff time that offers their better performance and T-Lohi uses the one given by the protocol specification and dependent on the maximum propagation time.
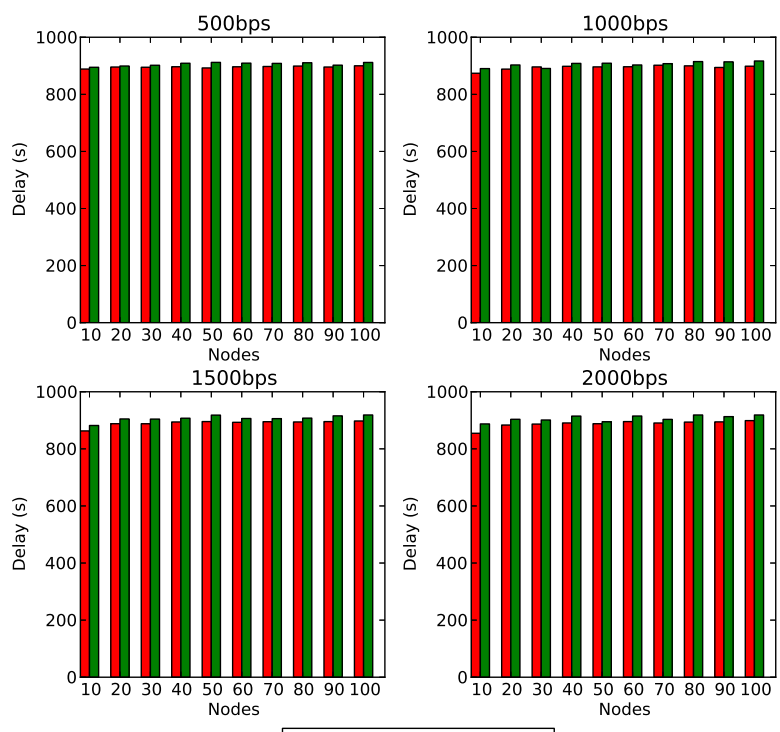

Figure A.45: Acknowledgements influence on delay Aloha 

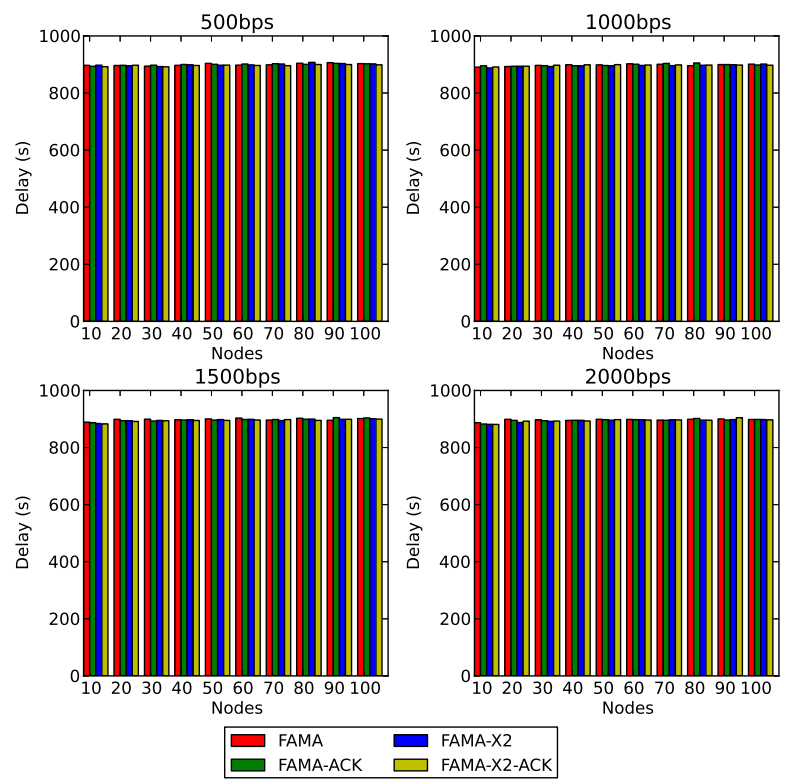

Figure A.46: Acknowledgements influence on delay FAMA
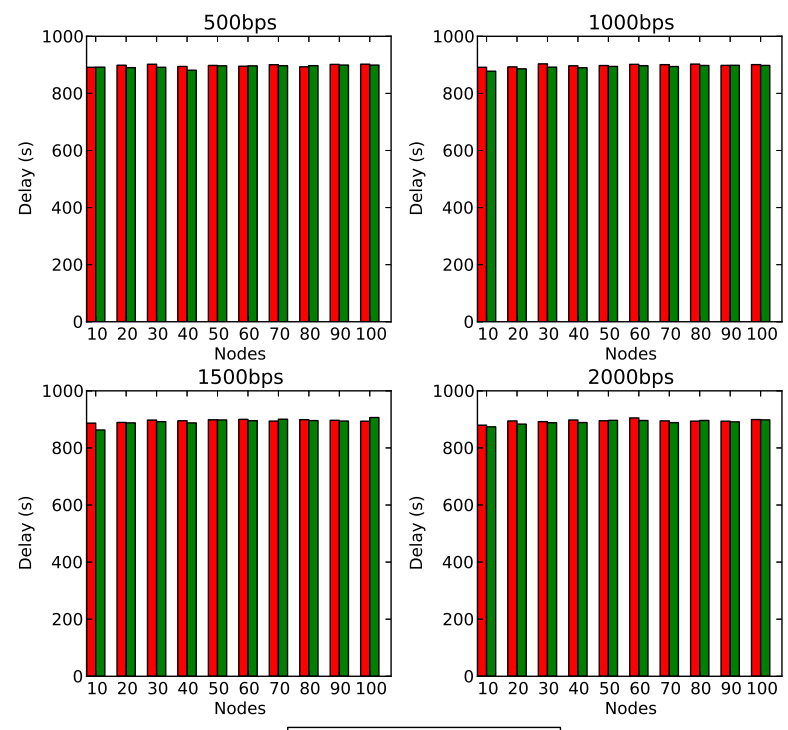

MACA $\square$ MACA-ACK

Figure A.47: Acknowledgements influence on delay MACA 

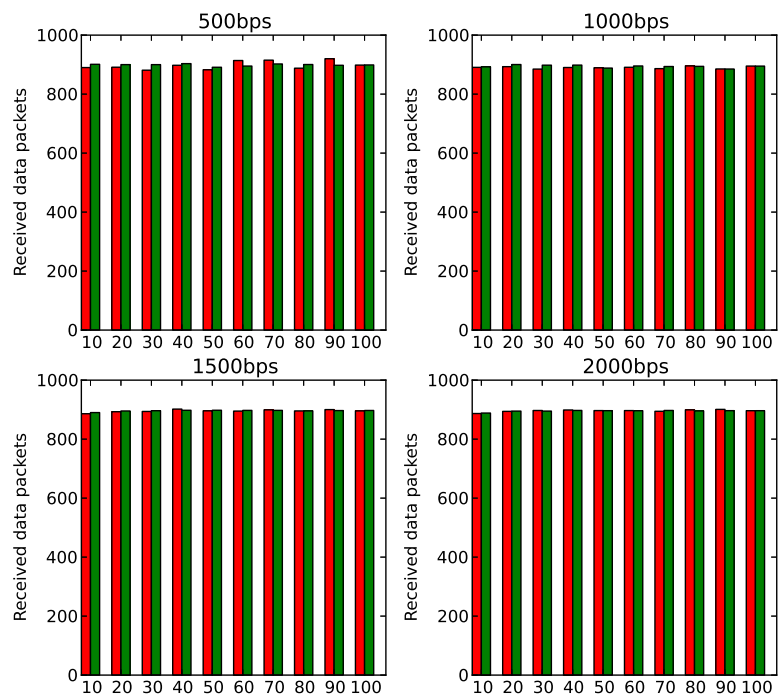

T-Lohi T-Lohi-ACK

Figure A.48: Acknowledgements influence on delay T-Lohi 


\section{A.8 Influence of acknowledgements on node's energy}

This section includes all the results regarding the influence of acknowledgements on node's energy consumption. As explained in Chapter 5, all protocols use the backoff time that offers their better performance and T-Lohi uses the one given by the protocol specification and dependent on the maximum propagation time.
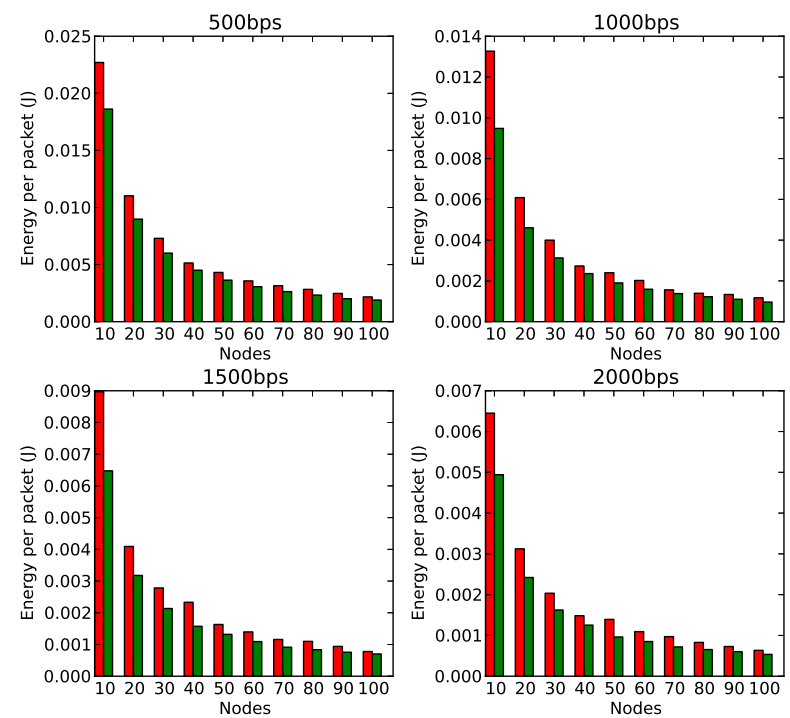

$\square$ MACA $\square$ MACA ACK

Figure A.49: Acknowledgements influence on nodes's energy - Aloha 

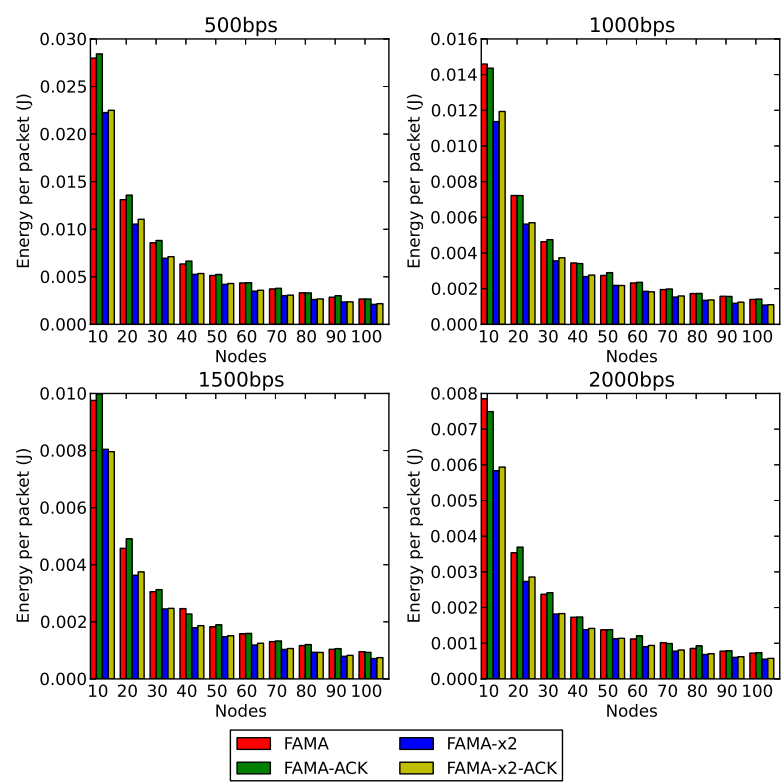

Figure A.50: Acknowledgements influence on nodes's energy - FAMA
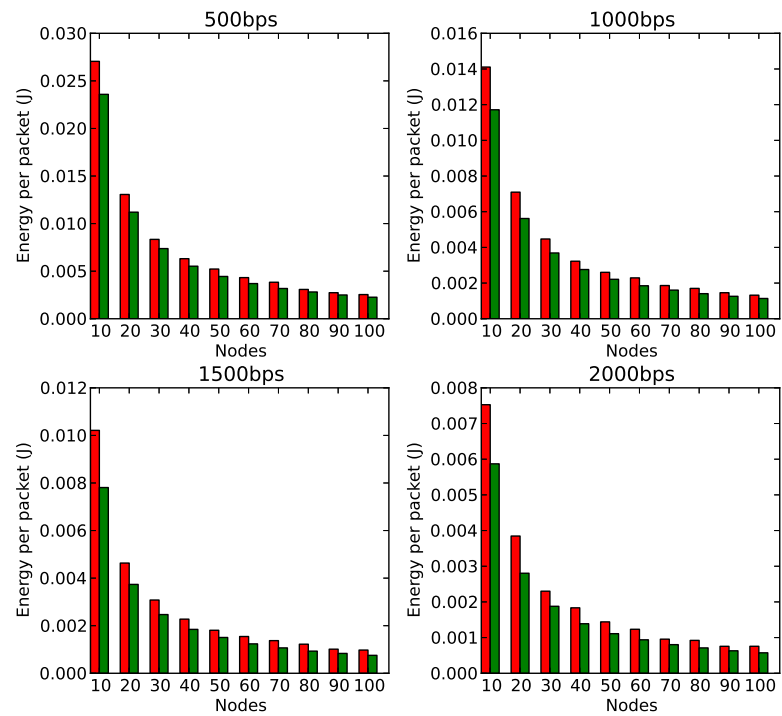

$\square$ MACA MACAACK

Figure A.51: Acknowledgements influence on nodes's energy - MACA 


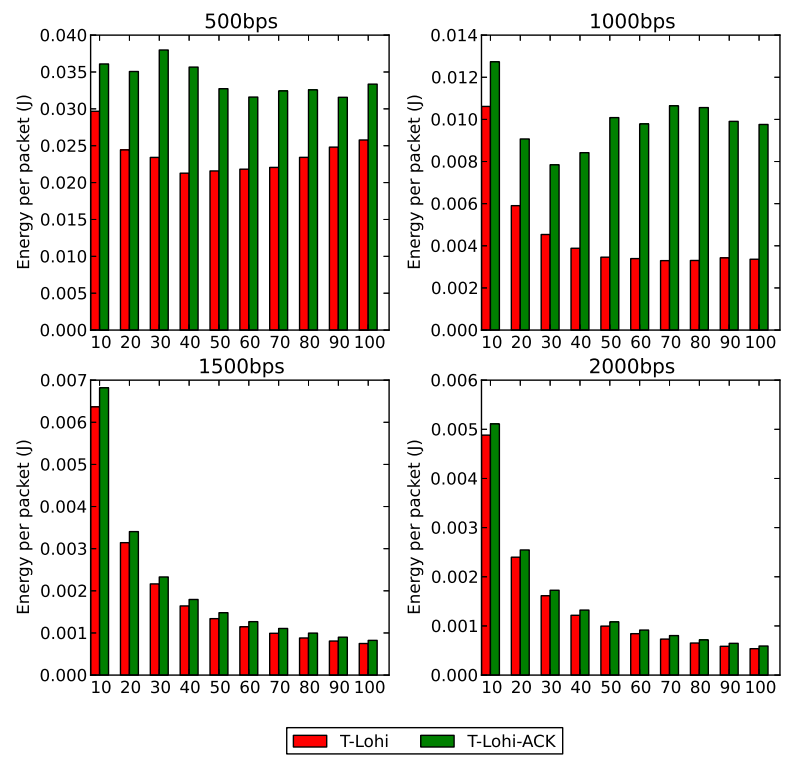

Figure A.52: Acknowledgements influence on nodes's energy - T-Lohi 


\section{A.9 Influence of acknowledgements on sink's energy}

This section includes all the results regarding the influence of acknowledgements on sink's energy consumption. As explained in Chapter 5, all protocols use the backoff time that offers their better performance and T-Lohi uses the one given by the protocol specification and dependent on the maximum propagation time.
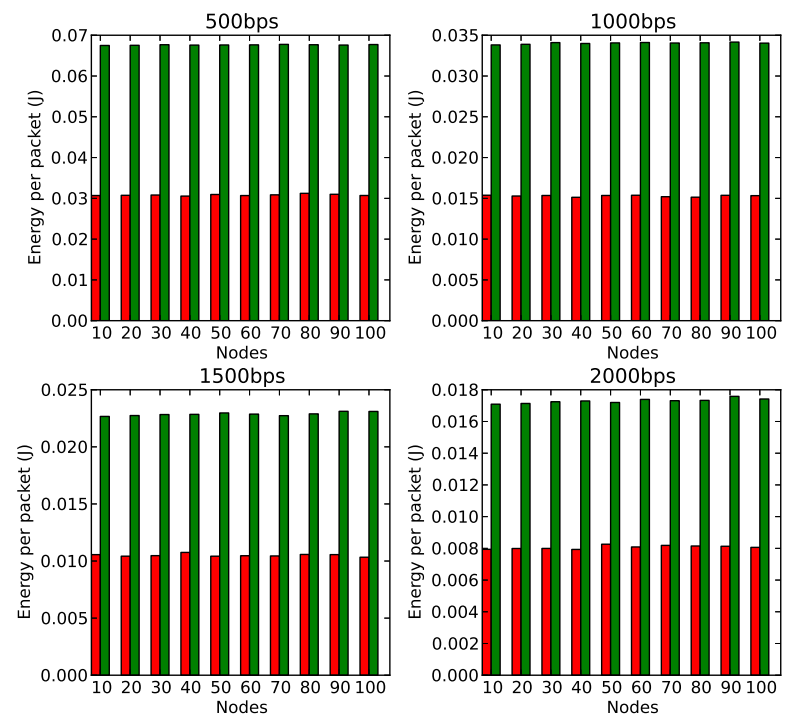

Aloha $\square$ Aloha ACK

Figure A.53: Acknowledgements influence on sink's energy - Aloha 

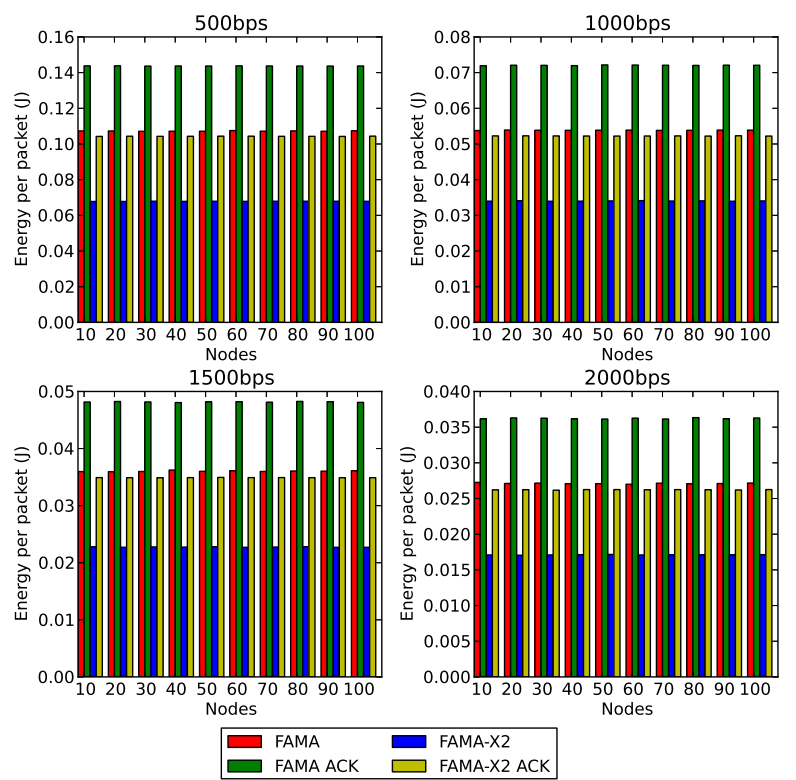

Figure A.54: Acknowledgements influence on sink's energy - FAMA
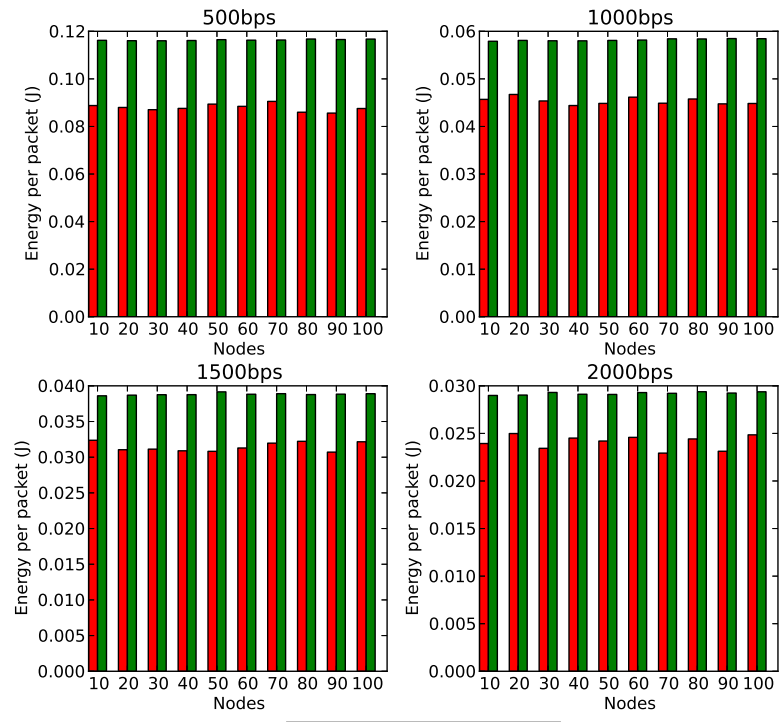

MACA MACA ACK

Figure A.55: Acknowledgements influence on sink's energy - MACA 

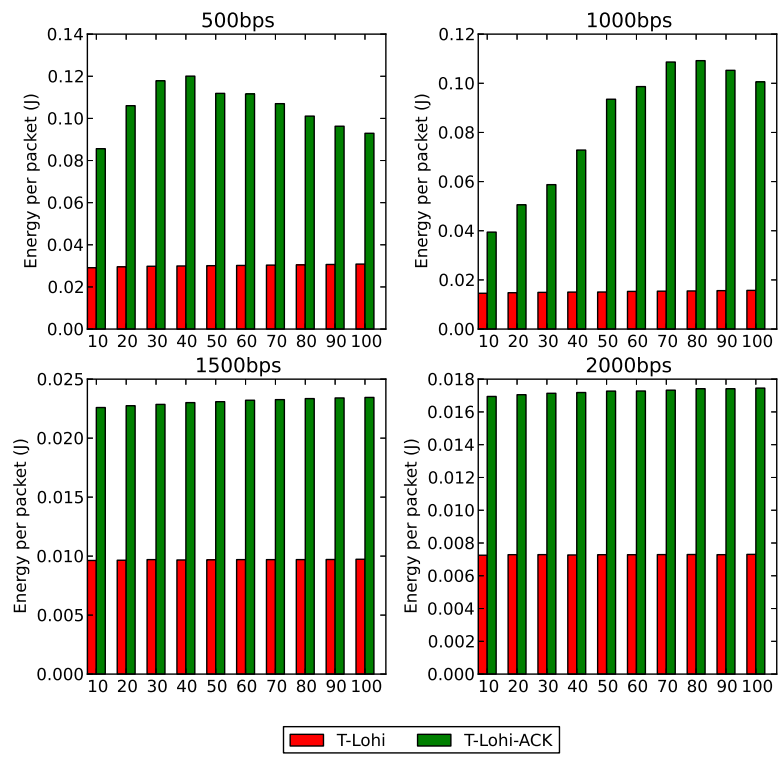

Figure A.56: Acknowledgements influence on sink's energy - T-Lohi 


\section{A.10 Influence of transmission speed on protocol throughput}

This section includes all the results regarding the influence of transmission speed on protocol throughput. As explained in Chapter 5, all protocols use the backoff time that offers their better performance and T-Lohi uses the one given by the protocol specification and dependent on the maximum propagation time.

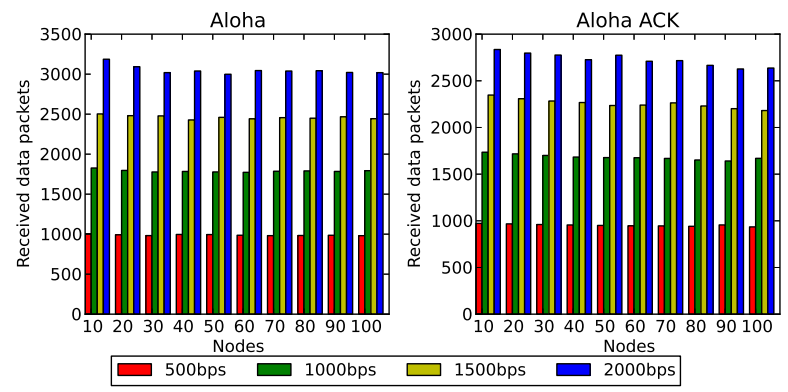

Figure A.57: Transmission speed influence on throughput Aloha

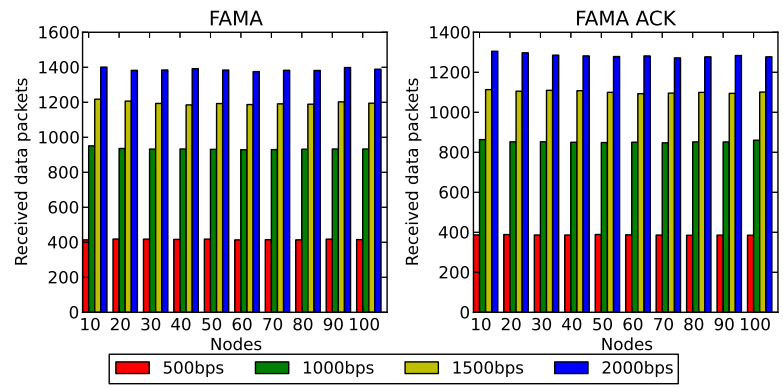

Figure A.58: Transmission speed influence on throughput FAMA 


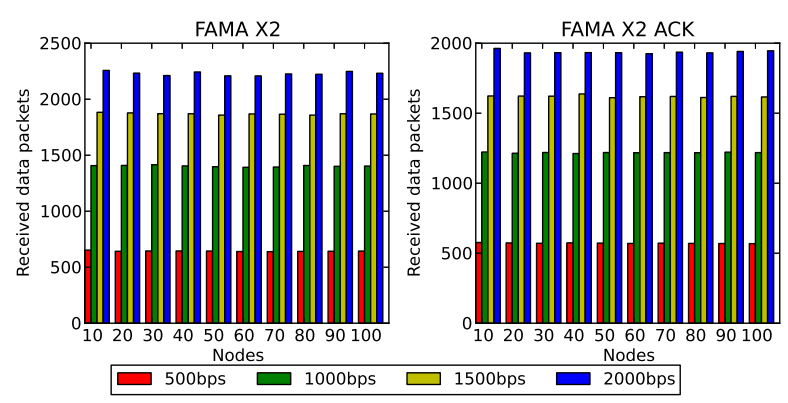

Figure A.59: Transmission speed influence on throughput FAMA X2

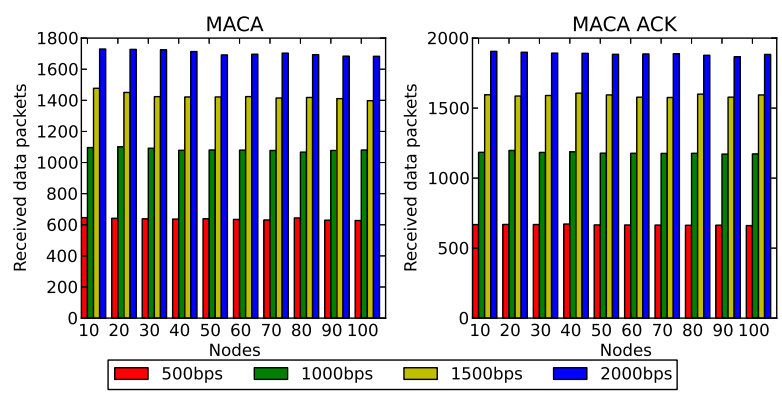

Figure A.60: Transmission speed influence on throughput MACA

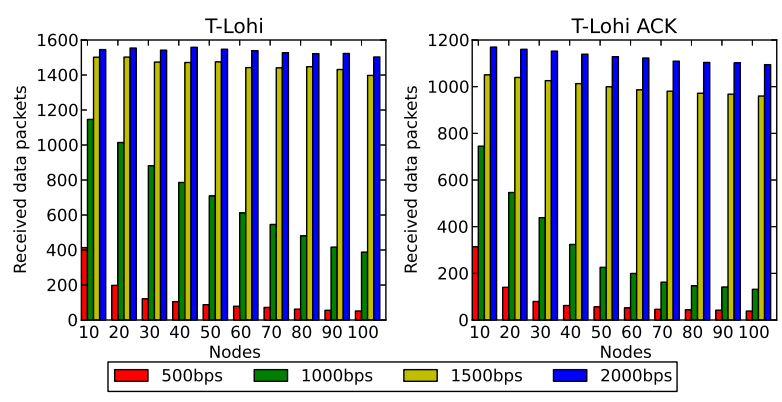

Figure A.61: Transmission speed influence on throughput T-Lohi 


\section{A.11 Influence of transmission speed on node energy consumption}

This section includes all the results regarding the influence of transmission speed on node's energy consumption. As explained in Chapter 5, all protocols use the backoff time that offers their better performance and T-Lohi uses the one given by the protocol specification and dependent on the maximum propagation time.

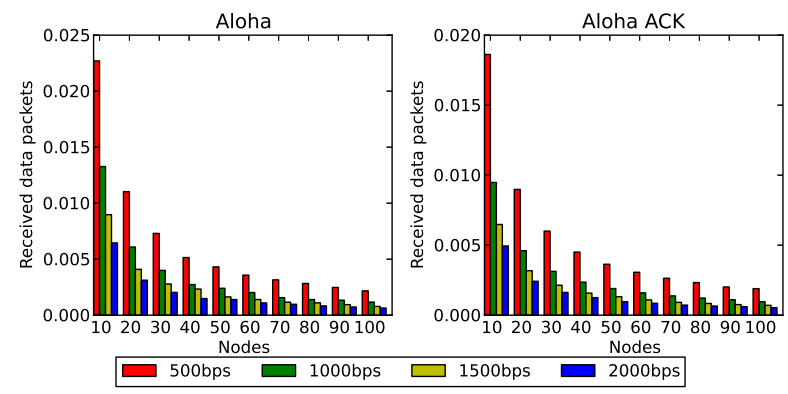

Figure A.62: Transmission speed influence on Aloha node energy consumption

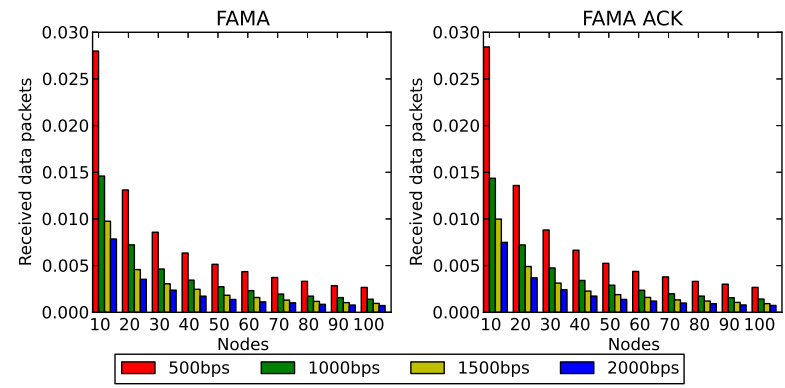

Figure A.63: Transmission speed influence on FAMA node energy consumption 


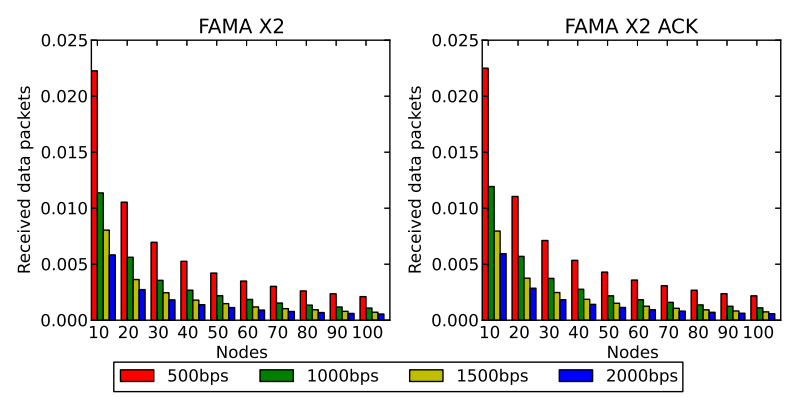

Figure A.64: Transmission speed influence on FAMA X2 node energy consumption

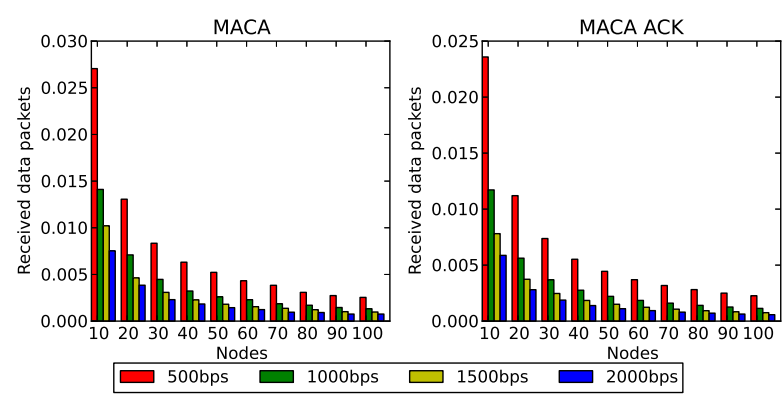

Figure A.65: Transmission speed influence on MACA node energy consumption

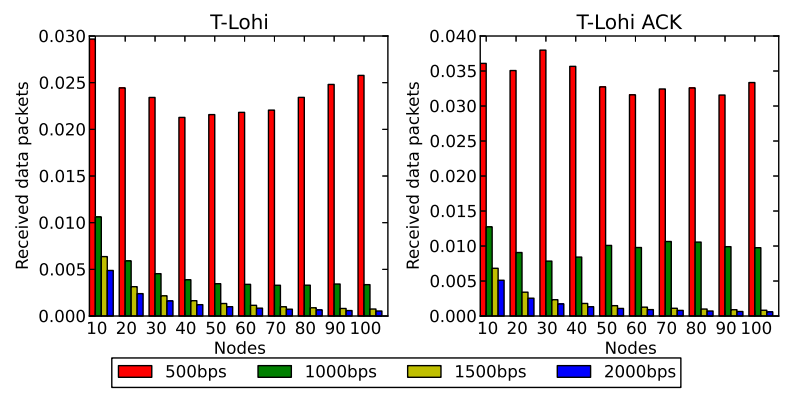

Figure A.66: Transmission speed influence on T-Lohi node energy consumption 


\section{A.12 Influence of transmission speed on sink energy consumption}

This section includes all the results regarding the influence of transmission speed on sink's energy consumption. As explained in Chapter 5, all protocols use the backoff time that offers their better performance and T-Lohi uses the one given by the protocol specification and dependent on the maximum propagation time.

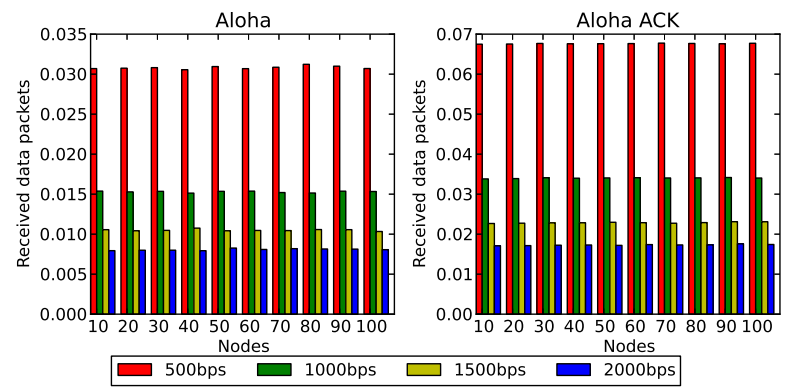

Figure A.67: Transmission speed influence on Aloha sink energy consumption

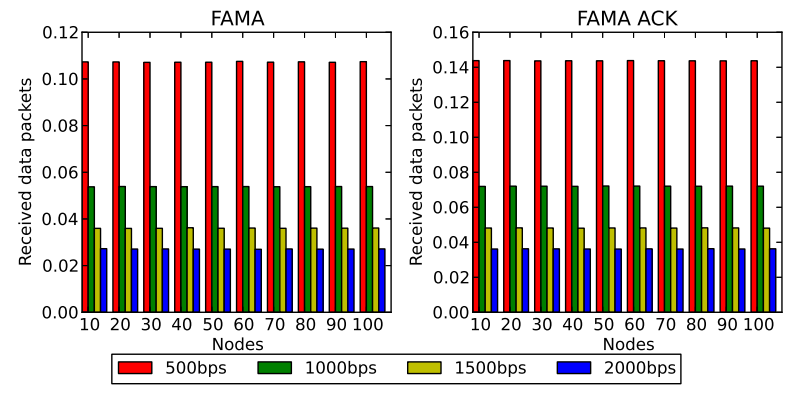

Figure A.68: Transmission speed influence on FAMA sink energy consumption 


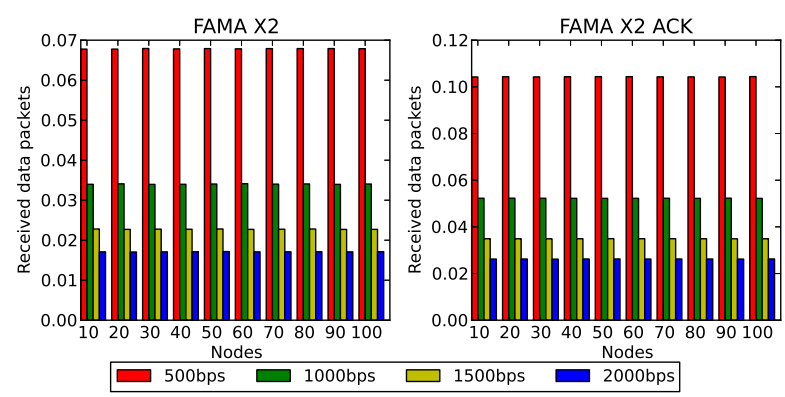

Figure A.69: Transmission speed influence on FAMA X2 sink energy consumption

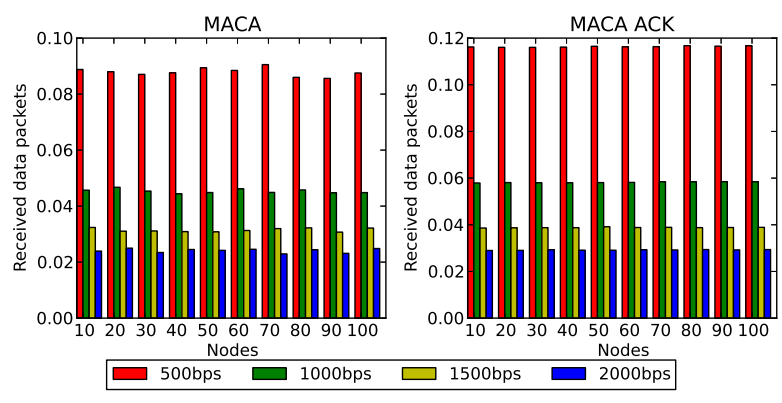

Figure A.70: Transmission speed influence on MACA sink energy consumption

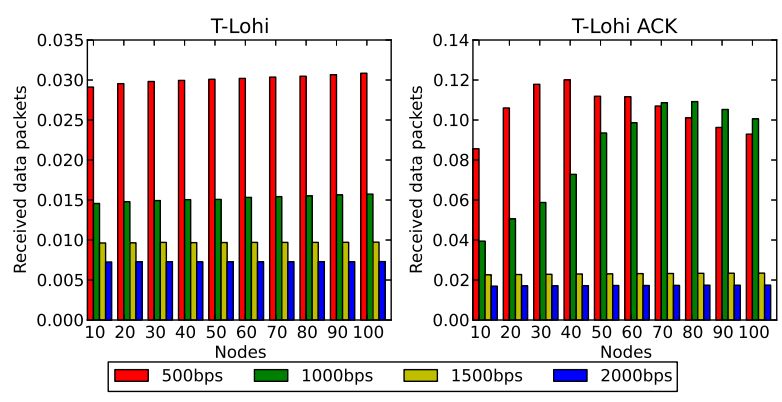

Figure A.71: Transmission speed influence on T-Lohi sink energy consumption 


\section{Bibliography}

[1] IERC. (2010). European Research Cluster on the Internet of Things, [Online]. Available: \%7Bhttp : / / www . internet - of - things - research . eu / about_iot.htm\%7D (visited on 09/2013) (cit. on p. 1).

[2] Libelium. (2013). 50 Sensor Applications for a Smarter World, [Online]. Available: http://www. libelium.com/top $\% 5$ C_50\%5C_iot $\% 5$ C_sensor $\%$ 5C_applications\%5C_ranking/ (visited on 09/2013) (cit. on p. 1).

[3] R. Headrick and L. Freitag, 'Growth of underwater communication technology in the U.S. Navy', English, IEEE Communications Magazine, vol. 47, no. 1, pp. 80-82, Jan. 2009, ISSN: 0163-6804 (cit. on p. 2).

[4] B. Benson, 'Design of a Low-cost Underwater Acoustic Modem for ShortRange Sensor Networking Appications', $\mathrm{PhD}$ thesis, University of California, San Diego, 2010 (cit. on p. 3).

[5] J. Heidemann, M. Stojanovic and M. Zorzi, 'Underwater sensor networks: applications, advances and challenges.', Philosophical transactions. Series A, Mathematical, physical, and engineering sciences, vol. 370, no. 1958, pp. 158-75, Jan. 2012, ISSN: 1364-503X (cit. on pp. 3, 10).

[6] I. F. Akyildiz, D. Pompili and T. Melodia, 'Underwater acoustic sensor networks: research challenges', Ad Hoc Networks, vol. 3, no. 3, pp. 257-279, May 2005, ISSN: 15708705 (cit. on pp. 3, 15, 48).

[7] M. Stojanovic and J. Preisig, 'Underwater acoustic communication channels: Propagation models and statistical characterization', IEEE Communications Magazine, vol. 47, no. 1, pp. 84-89, Jan. 2009, ISSN: 0163-6804 (cit. on pp. 3, 9). 
[8] Evologics. (nd). Underwater Acoustic Modem, [Online]. Available: www . evologics.de/en/products/acoustics/index.html (visited on 09/2013) (cit. on p. 3).

[9] Benthos Inc. (2013). Acoustic Modems, [Online]. Available: http://www . benthos.com/index.php/product_dashboard/acoustic_modems (visited on $09 / 2013$ ) (cit. on pp. 3,11).

[10] M. C. Domingo, 'Securing underwater wireless communication networks', Wireless Communications, IEEE, vol. 18, no. 1, pp. 22-28, 2011, ISSN: 15361284 (cit. on p. 9).

[11] A. A. Syed and J. Heidemann, 'Time Synchronization for High Latency Acoustic Networks', in INFOCOM 2006. 25th IEEE International Conference on Computer Communications. Proceedings, 2006, pp. 1-12 (cit. on pp. $9,17,86)$.

[12] H.-P. Tan, R. Diamant, W. K. G. Seah and M. Waldmeyer, 'A survey of techniques and challenges in underwater localization', Ocean Engineering, vol. 38 , no. $14-15$, pp. $1663-1676$, 2011, ISSN: 0029-8018 (cit. on pp. 9, 86).

[13] R. Otnes, A. Asterjadhi, P. Casari, M. Goetz, T. Husøy, I. Nissen, K. Rimstad, P. Walree and M. Zorzi, 'Medium Access Control', in Underwater Acoustic Networking Techniques, Springer Berlin Heidelberg, 2012, ch. 3, pp. 19-48, ISBN: 978-3-642-25223-5 (cit. on p. 9).

[14] M. Ayaz, I. Baig, A. Abdullah and I. Faye, 'A survey on routing techniques in underwater wireless sensor networks', Journal of Network and Computer Applications, vol. 34, no. 6, pp. 1908-1927, 2011 (cit. on pp. 9, 86).

[15] K. V. Mackenzie, 'Nine-term equation for sound speed in the oceans', en, The Journal of the Acoustical Society of America, vol. 70, no. 3, p. 807, Sep. 1981, ISSN: 00014966 (cit. on p. 10).

[16] L. Brekhovskikh and I. P. Lysanov, Fundamentals of Ocean Acoustics. Springer, 2003, p. 278, ISBN: 0387954678 (cit. on p. 10).

[17] M. Stojanovic, 'On the relationship between capacity and distance in an underwater acoustic communication channel', ACM SIGMOBILE Mobile Computing and Communications Review, vol. 11, no. 4, p. 34, Oct. 2007, ISSN: 15591662 (cit. on p. 10). 
[18] S. Basagni, M. Conti, S. Giordano and I. Stojmenovic, Eds., Mobile Ad Hoc Networking. Hoboken, NJ, USA: John Wiley \& Sons, Inc., Feb. 2013, ISBN: 9781118511305 (cit. on p. 10).

[19] X. Yu, 'Wireline quality underwater wireless communication using high speed acoustic modems', English, in OCEANS 2000 MTS/IEEE Conference and Exhibition. Conference Proceedings (Cat. No.00CH37158), vol. 1, IEEE, 2000, pp. 417-422, ISBN: 0-7803-6551-8 (cit. on p. 11).

[20] L. Freitag, M. Grund, S. Singh, J. Partan, P. Koski and K. Ball, 'The WHOI Micro-Modem: An Acoustic Communications and Navigation System for Multiple Platforms', in Proceedings of OCEANS 2005 MTS/IEEE, Washington, DC: IEEE, 2005, pp. 1086-1092, ISBN: 0-933957-34-3 (cit. on p. 11).

[21] B. Benson, Y. Li, R. Kastner, B. Faunce, K. Domond, D. Kimball and C. Schurgers, 'Design of a low-cost, underwater acoustic modem for shortrange sensor networks', in OCEANS'10 IEEE SYDNEY, IEEE, May 2010, pp. 1-9, ISBN: 978-1-4244-5221-7 (cit. on p. 11).

[22] Aquatec Group. (2013). AQUAmodem 500 Overview, [Online]. Available: http://www.aquatecgroup.com/ (visited on 10/2013) (cit. on p. 11).

[23] WHOI. (2013). Woods Hole Oceanographic Institution, [Online]. Available: http: //www. whoi.edu/ (visited on 09/2013) (cit. on p. 11).

[24] Tritech International. (2013). Tritech Mircon Data Modem - Small ROV Communications, [Online]. Available: http : / / www . tritech . co . uk / product/micron-data-modem (visited on 10/2013) (cit. on p. 11).

[25] LinkQuest Ink. (2013). Underwater acoustic modems, [Online]. Available: www.link-quest.com (cit. on p. 11).

[26] E. M. Sözer and M. Stojanovic, 'Reconfigurable acoustic modem for underwater sensor networks', in Proceedings of the 1st ACM international workshop on Underwater networks - WUWNet '06, New York, New York, USA: ACM Press, Sep. 2006, pp. 101-104, ISBN: 1595934847 (cit. on p. 11).

[27] H. Yan, S. Zhou, Z. J. Shi and B. Li, 'A DSP implementation of OFDM acoustic modem', in Proceedings of the second workshop on Underwater networks - WuWNet '07, New York, New York, USA: ACM Press, Sep. 2007, pp. 89-92, ISBN: 9781595937360 (cit. on p. 11). 
[28] A. Sanchez, S. Blanc, P. Yuste, A. Perles and J. J. Serrano, 'An Ultra-Low Power and Flexible Acoustic Modem Design to Develop Energy-Efficient Underwater Sensor Networks', Sensors, vol. 12, no. 6, pp. 6837-6856, 2012 (cit. on pp. 11, 25, 28, 32, 49).

[29] C. Schurgers, V. Tsiatsis, S. Ganeriwal and M. Srivastava, 'Optimizing sensor networks in the energy-latency-density design space', IEEE Transactions on Mobile Computing, vol. 1, no. 1, pp. 70-80, Jan. 2002, ISSN: 1536-1233 (cit. on p. 11).

[30] J. Wills, W. Ye and J. Heidemann, 'Low-power acoustic modem for dense underwater sensor networks', in WUWNet '06 Proceedings of the 1st ACM international workshop on Underwater networks, 2006, pp. 79-85 (cit. on pp. 11, 25, 28, 31, 32).

[31] A. Sanchez, S. Blanc, P. Yuste, I. Piqueras, J. Jos\&\#233 and Serrano, 'Advanced Acoustic Wake-up System for Underwater Sensor Networks', en, Communications in Information Science and Management Engineering, vol. 2, no. 2, pp. 1-10, Feb. 2012, ISSN: 1945-3194 (cit. on p. 11).

[32] A. A. Syed, W. Ye, J. Heidemann and B. Krishnamachari, 'Understanding spatio-temporal uncertainty in medium access with ALOHA protocols', in Proceedings of the second workshop on Underwater networks WuWNet '07, New York, New York, USA: ACM Press, Sep. 2007, p. 41, ISBN: 9781595937360 (cit. on pp. 12, 17).

[33] J. Rice, B. Creber, C. Fletcher, P. Baxley, K. Rogers, K. McDonald, D. Rees, M. Wolf, S. Merriam, R. Mehio, J. Proakis, K. Scussel, D. Porta, J. Baker, J. Hardiman and D. Green, 'Evolution of Seaweb underwater acoustic networking', in OCEANS 2000 MTS/IEEE Conference and Exhibition, vol. 3, IEEE, 2000, pp. 2007-2017, ISBN: 0-7803-6551-8 (cit. on pp. 13, 14).

[34] D. Pompili, T. Melodia and I. F. Akyildiz, 'A CDMA-based medium access control for underwater acoustic sensor networks', IEEE Transactions on Wireless Communications, vol. 8, no. 4, pp. 1899-1509, Apr. 2009, ISSN: 1536-1276 (cit. on pp. 14, 16).

[35] M. K. Watfa, S. Selman and H. Denkilkian, 'UW-MAC: An underwater sensor network MAC protocol', International Journal of Communication Systems, vol. 23, no. 4, pp. 485-506, Apr. 2010, ISSN: 10745351 (cit. on pp. 14, 16). 
[36] S. Climent, J. Capella, N. Meratnia and J. Serrano, 'Underwater Sensor Networks: A New Energy Efficient and Robust Architecture', Sensors, vol. 12, no. 1, pp. 704-731, 2012 (cit. on pp. 14, 16, 87).

[37] G. Fan, H. Chen, L. Xie and K. Wang, 'A hybrid reservation-based MAC protocol for underwater acoustic sensor networks', Ad Hoc Networks, Jan. 2013, ISSN: 15708705 (cit. on pp. 14, 16).

[38] C.-C. Hsu, K.-F. Lai, C.-F. Chou and K. C.-J. Lin, 'ST-MAC: SpatialTemporal MAC Scheduling for Underwater Sensor Networks', in IEEE INFOCOM 2009 - The 28th Conference on Computer Communications, IEEE, Apr. 2009, pp. 1827-1835 (cit. on pp. 14, 18).

[39] K. Kredo II and P. Mohapatra, 'Distributed Scheduling and Routing in Underwater Wireless Networks', in 2010 IEEE Global Telecommunications Conference GLOBECOM 2010, IEEE, Dec. 2010, pp. 1-6 (cit. on pp. 14, 18).

[40] W. Van Kleunen, N. Meratnia and P. J. Havinga, 'MDS-Mac: A Scheduled MAC for Localization, Time-Synchronisation and Communication in Underwater Acoustic Networks', in 2012 IEEE 15th International Conference on Computational Science and Engineering, IEEE, Dec. 2012, pp. 666-672, ISBN: 978-1-4673-5165-2 (cit. on pp. 14, 18, 23).

[41] W. van Kleunen, N. Meratnia and P. Havinga, 'MAC Scheduling in Largescale Underwater Acoustic Networks', SciTePress, Jul. 2011 (cit. on pp. 14, 18).

[42] A. S. Tanenbaum and D. J. Wetherall, Computer Networks. Prentice Hall, 2010, p. 933, ISBN: 0132126958 (cit. on pp. 14, 19, 20).

[43] J. Ahn, A. Syed, B. Krishnamachari and J. Heidemann, 'Design and analysis of a propagation delay tolerant ALOHA protocol for underwater networks', Ad Hoc Networks, vol. 9, no. 5, pp. 752-766, Jul. 2011, ISSN: 15708705 (cit. on pp. 14, 19).

[44] M. Molins and M. Stojanovic, 'Slotted FAMA: a MAC protocol for underwater acoustic networks', in OCEANS 2006-Asia Pacific, 2007, pp. 1-7 (cit. on pp. 14, 19, 22).

[45] R. Diamant and L. Lampe, 'Spatial Reuse Time-Division Multiple Access for Broadcast Ad Hoc Underwater Acoustic Communication Networks', 
IEEE Journal of Oceanic Engineering, vol. 36, no. 2, pp. 172-185, Apr. 2011, ISSN: 0364-9059 (cit. on pp. 14, 19).

[46] M. K. Park and V. Rodoplu, 'UWAN-MAC: An Energy-Efficient MAC Protocol for Underwater Acoustic Wireless Sensor Networks', IEEE Journal of Oceanic Engineering, vol. 32, no. 3, pp. 710-720, Jul. 2007, ISSN: 03649059 (cit. on pp. 14, 19).

[47] Y. Zhu, Z. Jiang, Z. Peng, M. Zuba, J. Cui and H. Chen, 'Toward Practical MAC Design for Underwater Acoustic Networks', in IEEE INFOCOM, 2013, 2013 (cit. on pp. 14, 19).

[48] Y.-J. Chen and H.-L. Wang, 'Ordered CSMA: a collision-free MAC protocol for underwater acoustic networks', in Oceans 2007, IEEE, 2007, pp. 1-6 (cit. on pp. 14, 18).

[49] H.-H. Ng, W.-S. Soh and M. Motani, 'MACA-U: A Media Access Protocol for Underwater Acoustic Networks', in IEEE GLOBECOM 2008 - 2008 IEEE Global Telecommunications Conference, IEEE, 2008, pp. 1-5, ISBN: 978-1-4244-2324-8 (cit. on pp. 14, 21).

[50] X. Guo, 'A propagation-delay-tolerant collision avoidance protocol for underwater acoustic sensor networks', in OCEANS 2006-Asia Pacific, 2007, pp. 1-6 (cit. on pp. 14, 21).

[51] W.-H. Liao and C.-C. Huang, 'SF-MAC: A Spatially Fair MAC Protocol for Underwater Acoustic Sensor Networks', IEEE Sensors Journal, vol. 12, no. 6, pp. 1686-1694, Jun. 2012, ISSN: 1530-437X (cit. on pp. 14, 21).

[52] B. Peleato and M. Stojanovic, 'Distance aware collision avoidance protocol for ad-hoc underwater acoustic sensor networks', IEEE Communications Letters, vol. 11, no. 12, pp. 1025-1027, Dec. 2007, ISSN: 1089-7798 (cit. on pp. 14, 21).

[53] C. L. Fullmer and J. J. Garcia-Luna-Aceves, 'Floor acquisition multiple access (FAMA) for packet-radio networks', SIGCOMM Comput. Commun. Rev., vol. 25, no. 4, pp. 262-273, 1995 (cit. on pp. 14, 19, 22).

[54] Z. Peng, Z. Zhou, Z. Guo and J.-H. Cui, 'COPE-MAC: A Contentionbased medium access control protocol with Parallel Reservation for underwater acoustic networks', in OCEANS'10 IEEE SYDNEY, IEEE, May 2010, pp. 1-10, ISBN: 978-1-4244-5221-7 (cit. on pp. 14, 22). 
[55] P. Xie and J.-H. Cui, 'R-MAC: An Energy-Efficient MAC Protocol for Underwater Sensor Networks', in International Conference on Wireless Algorithms, Systems and Applications (WASA 2007), IEEE, Aug. 2007, pp. 187198, ISBN: 0-7695-2981-X (cit. on pp. 14, 22).

[56] Y. Noh, P. Wang, U. Lee, D. Torres and M. Gerla, 'DOTS: A propagation Delay-aware Opportunistic MAC protocol for underwater sensor networks', in The 18th IEEE International Conference on Network Protocols, Kyoto: IEEE, Oct. 2010, pp. 183-192, ISBN: 978-1-4244-8644-1 (cit. on pp. 14, 22).

[57] N. Chirdchoo, W.-s. Soh and K. Chua, 'RIPT: A Receiver-Initiated ReservationBased Protocol for Underwater Acoustic Networks', IEEE Journal on Selected Areas in Communications, vol. 26, no. 9, pp. 1744-1753, Dec. 2008, ISSN: 0733-8716 (cit. on pp. 14, 22).

[58] A. Syed, W. Ye and J. Heidemann, 'T-Lohi: A new class of MAC protocols for underwater acoustic sensor networks', in INFOCOM 2008. The 27th Conference on Computer Communications. IEEE, IEEE, 2008, pp. 231235 (cit. on pp. 14, 22, 23).

[59] E. Sozer, M. Stojanovic and J. Proakis, 'Underwater acoustic networks', IEEE Journal of Oceanic Engineering, vol. 25, no. 1, pp. 72-83, Jan. 2000, ISSN: 0364-9059 (cit. on pp. 15, 48).

[60] M. Stojanovic and L. Freitag, 'Multichannel Detection for Wideband Underwater Acoustic CDMA Communications', IEEE Journal of Oceanic Engineering, vol. 31, no. 3, pp. 685-695, Jul. 2006, ISSN: 0364-9059 (cit. on p. 15).

[61] K. Kredo II, P. Djukic and P. Mohapatra, 'STUMP: Exploiting Position Diversity in the Staggered TDMA Underwater MAC Protocol', in IEEE INFOCOM 2009 - The 28th Conference on Computer Communications, IEEE, Apr. 2009, pp. 2961-2965, ISBN: 978-1-4244-3512-8 (cit. on p. 18).

[62] Y. Xiao, Y. Zhang, J. Gibson, G. Xie and H. Chen, 'Performance analysis of ALOHA and p-persistent ALOHA for multi-hop underwater acoustic sensor networks', Cluster Computing, vol. 14, no. 1, pp. 65-80, 2011, ISSN: 1386-7857 (cit. on p. 20).

[63] N. Chirdchoo, W. Soh and K. Chua, 'Aloha-based MAC protocols with collision avoidance for underwater acoustic networks', in INFOCOM $200 \%$. 26th IEEE International Conference on Computer Communications. IEEE, 2007, pp. 2271-2275 (cit. on p. 20). 
[64] P. Karn, 'MACA - a new channel access method for packet radio', in ARRL/CRRL Amateur Radio 9th Computer Networking Conference, 1990, pp. 134-140 (cit. on p. 21).

[65] A. F. Harris III, M. Stojanovic and M. Zorzi, 'Idle-time energy savings through wake-up modes in underwater acoustic networks', Ad Hoc Networks, vol. 7, no. 4, pp. 770-777, 2009 (cit. on p. 25).

[66] Ns-3. (nd). Ns-3, [Online]. Available: http://www . nsnam.org (visited on 06/2013) (cit. on pp. 25, 26, 49).

[67] Aqua-Sim. (2013). Aqua-Sim, [Online]. Available: http://obinet.engr . uconn.edu/ (visited on 06/2013) (cit. on p. 25).

[68] Ns-miracle. (nd). NS-Miracle, [Online]. Available: http://telecom.dei . unipd.it (visited on 06/2013) (cit. on p. 25).

[69] M. B. Porter and H. P. Bucker, 'Gaussian beam tracing for computing ocean acoustic fields', en, The Journal of the Acoustical Society of America, vol. 82, no. 4, pp. 1349-1359, Oct. 1987, ISSN: 00014966 (cit. on p. 27).

[70] Oalib. (nd). Ocean Acoustics Library, [Online]. Available: oalib.hlsresearch. com (visited on 06/2013) (cit. on p. 27).

[71] F. Guerra, P. Casari and M. Zorzi, 'World Ocean Simulation System (WOSS): a simulation tool for underwater networks with realistic propagation modeling', in Proceedings of the Fourth ACM International Workshop on UnderWater Networks, ACM, 2009, 4:1-4:8 (cit. on pp. 27, 49).

[72] GEBCO. (2013). General Bathymetric Chart of the Oceans, [Online]. Available: www.gebco.net (visited on 06/2013) (cit. on p. 28).

[73] NOAA. (2013). National Oceanic and Atmospheric Administration, [Online]. Available: www.noaa.gov (visited on 06/2013) (cit. on p. 28).

[74] MICAz. (nd). Wireless Measurement System, [Online]. Available: http: //www . openautomation.net/uploadsproductos/micaz_datasheet.pdf (visited on 06/2013) (cit. on pp. 28, 32).

[75] A. Microsystems. (2013). AS3933 - 3D Low Frequency Wakeup Receiver, [Online]. Available: www . austriamicrosystems . com (visited on 06/2013) (cit. on p. 28). 
[76] TI. (2013). Analog, Embedded Processing, Semiconductor Company, Texas Instruments, [Online]. Available: www.ti.com (visited on 06/2013) (cit. on pp. $32,41,42)$.

[77] R. Vullers, R. Schaijk, H. Visser, J. Penders and C. Hoof, 'Energy Harvesting for Autonomous Wireless Sensor Networks', IEEE Solid-State Circuits Magazine, vol. 2, no. 2, pp. 29-38, 2010, ISSN: 1943-0582 (cit. on p. 35).

[78] W. Seah, Y. K. Tan and A. Chan, 'Research in Energy Harvesting Wireless Sensor Networks and the Challenges Ahead', English, in Autonomous Sensor Networks SE - 27, ser. Springer Series on Chemical Sensors and Biosensors, D. Filippini, Ed., vol. 13, Springer Berlin Heidelberg, 2013, pp. 73 93, ISBN: 978-3-642-34647-7 (cit. on p. 35).

[79] G. Merrett, A. Weddell, A. Lewis, N. Harris, B. Al-Hashimi and N. White, 'An Empirical Energy Model for Supercapacitor Powered Wireless Sensor Nodes', in 2008 Proceedings of 17th International Conference on Computer Communications and Networks, IEEE, Aug. 2008, pp. 1-6, ISBN: 978-14244-2389-7 (cit. on p. 35).

[80] V. Raghunathan, A. Kansal, J. Hsu, J. Friedman and M. Srivastava, 'Design considerations for solar energy harvesting wireless embedded systems', in IPSN '05 Proceedings of the 4th international symposium on Information processing in sensor networks, Los Angeles, California: IEEE Press, Apr. 2005, p. 64, ISBN: 0-7803-9202-7 (cit. on p. 35).

[81] K. Zeng, K. Ren, W. Lou and P. J. Moran, 'Energy aware efficient geographic routing in lossy wireless sensor networks with environmental energy supply', Wireless Networks, vol. 15, no. 1, pp. 39-51, Feb. 2007, ISSN: 1022-0038 (cit. on p. 35).

[82] D. Hasenfratz, A. Meier, C. Moser, J.-J. Chen and L. Thiele, 'Analysis, Comparison, and Optimization of Routing Protocols for Energy Harvesting Wireless Sensor Networks', in 2010 IEEE International Conference on Sensor Networks, Ubiquitous, and Trustworthy Computing, IEEE, 2010, pp. 19-26, ISBN: 978-1-4244-7087-7 (cit. on p. 35).

[83] D. K. Noh and J. Hur, 'Using a dynamic backbone for efficient data delivery in solar-powered WSNs', Journal of Network and Computer Applications, vol. 35, no. 4, pp. 1277-1284, Jul. 2012, ISSN: 10848045 (cit. on p. 35).

[84] L. Lin, N. B. Shroff and R. Srikant, 'Asymptotically Optimal Energy-Aware Routing for Multihop Wireless Networks With Renewable Energy Sources', 
IEEE/ACM Transactions on Networking, vol. 15, no. 5, pp. 1021-1034, Oct. 2007, ISSN: 1063-6692 (cit. on p. 35).

[85] N. Ferry, S. Ducloyer, N. Julien and D. Jutel, 'Power/energy estimator for designing WSN nodes with ambient energy harvesting feature', EURASIP J. Embedded Syst., vol. 2011, 6:1-6:17, 2011 (cit. on p. 35).

[86] J. Glaser, D. Weber, S. Madani and S. Mahlknecht, 'Power Aware Simulation Framework for Wireless Sensor Networks and Nodes', en, EURASIP Journal on Embedded Systems, vol. 2008, no. 1, p. 369 178, Jun. 2008, ISSN: 1687-3963 (cit. on p. 35).

[87] G. V. Merrett, N. M. White, N. R. Harris and B. M. Al-Hashimi, 'EnergyAware Simulation for Wireless Sensor Networks', in 2009 6th Annual IEEE Communications Society Conference on Sensor, Mesh and Ad Hoc Communications and Networks, Rome: IEEE, Jun. 2009, pp. 64-71, ISBN: 9781-4244-2907-3 (cit. on pp. 35, 37).

[88] P. De Mil, B. Jooris, L. Tytgat, R. Catteeuw, I. Moerman, P. Demeester and A. Kamerman, 'Design and Implementation of a Generic Energy-Harvesting Framework Applied to the Evaluation of a Large-Scale Electronic ShelfLabeling Wireless Sensor Network', EURASIP Journal on Wireless Communications and Networking, vol. 2010, no. 1, 7:1-7:12, Feb. 2010, ISSN: 1687-1499 (cit. on p. 36).

[89] A. Castagnetti, 'A framework for modeling and simulating energy harvesting WSN nodes with efficient power management policies', EURASIP J. Embedded Syst., vol. 2012, no. 1, p. 8, 2012 (cit. on p. 36).

[90] Y. Ammar, A. Buhrig, M. Marzencki, B. Charlot, S. Basrour, K. Matou and M. Renaudin, 'Wireless sensor network node with asynchronous architecture and vibration harvesting micro power generator', in Proceedings of the 2005 joint conference on Smart objects and ambient intelligence innovative context-aware services: usages and technologies - sOc-EUSAI '05, New York, New York, USA: ACM Press, Oct. 2005, p. 287, ISBN: 1595933042 (cit. on p. 36).

[91] K. Vijayaraghavan and R. Rajamani, 'Active Control Based Energy Harvesting for Battery-Less Wireless Traffic Sensors', in 2007 American Control Conference, IEEE, Jul. 2007, pp. 3106-3111, ISBN: 1-4244-0988-8 (cit. on p. 36). 
[92] L. Mateu, C. Codrea, N. Lucas, M. Pollak and P. Spies, 'Energy harvesting for wireless communication systems using thermogenerators', in Conference on Design of Circuits and Integrated Systems (DCIS), 2006 (cit. on p. 36).

[93] H. Bottner, J. Nurnus, A. Gavrikov, G. Kuhner, M. Jagle, C. Kunzel, D. Eberhard, G. Plescher, A. Schubert and K.-H. Schlereth, 'New thermoelectric components using microsystem technologies', Journal of Microelectromechanical Systems, vol. 13, no. 3, pp. 414-420, Jun. 2004, ISSN: 10577157 (cit. on p. 36).

[94] AEMet. (nd). Agencia Estatal de Meteorolgía, [Online]. Available: www . aemet.es (visited on 06/2013) (cit. on pp. 36, 41).

[95] PANGAEA. (nd). Data Publisher for Earth \& Environmental Science, [Online]. Available: http : / / www . pangaea.de/ (visited on 06/2013) (cit. on pp. $36,41,74)$.

[96] A. Sanchez, S. Climent, S. Blanc, J. V. Capella and I. Piqueras, 'WSN with energy-harvesting: modeling and simulation based on a practical architecture using real radiation levels', in Proceedings of the 6th ACM workshop on Performance monitoring and measurement of heterogeneous wireless and wired networks - PM2HW2N '11, New York, New York, USA: ACM Press, Oct. 2011, pp. 17-24, ISBN: 9781450309028 (cit. on pp. 37, 41, 89).

[97] A. Kansal, J. Hsu, S. Zahedi and M. B. Srivastava, 'Power management in energy harvesting sensor networks', ACM Trans. Embed. Comput. Syst., vol. 6, no. 4, Sep. 2007, ISSN: 1539-9087 (cit. on p. 37).

[98] T. Zhu, Z. Zhong, Y. Gu, T. He and Z.-L. Zhang, 'Leakage-aware energy synchronization for wireless sensor networks', in Proceedings of the 7th international conference on Mobile systems, applications, and services, ser. MobiSys '09, New York, NY, USA: ACM, 2009, pp. 319-332, ISBN: 978-160558-566-6 (cit. on p. 37).

[99] C. Renner, J. Jessen and V. Turau, 'Lifetime prediction for supercapacitorpowered wireless sensor nodes', Proc. of the 8th GI/ITG KuVS Fachgespräch" Drahtlose Sensornetze"(FGSN'09), Hamburg, Germany, 2009 (cit. on p. 37).

[100] F. P. Kelly and I. M. MacPhee, 'The number of packets transmitted by collision detect random access schemes', The Annals of Probability, pp. 15571568, 1987 (cit. on pp. 51, 57). 
[101] K.-W. Fan, Z. Zheng and P. Sinha, 'Steady and fair rate allocation for rechargeable sensors in perpetual sensor networks', in Proceedings of the 6th ACM conference on Embedded network sensor systems - SenSys '08, Raleigh, NC, USA: ACM Press, Nov. 2008, pp. 239-252, ISBN: 9781595939906 (cit. on p. 78).

[102] C. Moser, L. Thiele, D. Brunelli and L. Benini, 'Adaptive Power Management for Environmentally Powered Systems', IEEE Transactions on Computers, vol. 59, no. 4, pp. 478-491, Apr. 2010, ISSN: 0018-9340 (cit. on p. 78).

[103] B. Zhang, R. Simon and H. Aydin, 'Maximum utility rate allocation for energy harvesting wireless sensor networks', in Proceedings of the 14 th ACM international conference on Modeling, analysis and simulation of wireless and mobile systems - MSWiM '11, Miami, Florida, USA: ACM Press, Oct. 2011, pp. 7-16, ISBN: 9781450308984 (cit. on p. 78).

[104] F. Ferrari, A. Meier and L. Thiele, 'Accurate clock models for simulating Wireless Sensor Networks', in Proceedings of the 3rd International ICST Conference on Simulation Tools and Techniques, ICST (Institute for Computer Sciences, Social-Informatics and Telecommunications Engineering), 2010, pp. 1-4 (cit. on p. 86).

[105] A. Sanchez, S. Blanc, S. Climent, P. Yuste and R. Ors, 'SIVEH: Numerical Computing Simulation of Wireless Energy-Harvesting Sensor Nodes', Sensors, vol. 13, no. 9, pp. 11750-11771, 2013 (cit. on pp. 86, 88).

[106] J.-H. Cui, J. Kong, M. Gerla and S. Zhou, 'The challenges of building mobile underwater wireless networks for aquatic applications', Network, IEEE, vol. 20, no. 3, pp. 12-18, 2006, ISSN: 0890-8044 (cit. on p. 86).

[107] S. Climent, J. V. Capella, S. Blanc, A. Perles and J. J. Serrano, 'A Proposal for Modeling Real Hardware, Weather and Marine Conditions for Underwater Sensor Networks', Sensors, vol. 13, no. 6, pp. 7454-7471, 2013, ISSN: 1424-8220 (cit. on p. 87).

[108] S. Climent, A. Sanchez, S. Blanc, J. V. Capella and R. Ors, 'Wireless sensor network with energy harvesting: modeling and simulation based on a practical architecture using real radiation levels', Concurrency and Computation: Practice and Experience, no. In press, pp. 1-22, 2013 (cit. on p. 88).

[109] S. Climet, A. Sanchez, J. V. Capella, N. Meratnia and J. J. Serrano, 'Underwater Acoustic Wireless Sensor Networks: Advances and Future Trends', Sensors, no. In press, pp. 1-39, 2013 (cit. on p. 88). 
[110] S. Climent, J. Capella, A. Bonastre and R. Ors, 'A new model for the ns-3 simulator of a novel routing protocol applied to underwater WSN', Proceedings of the 2011 International Conference on Wireless Networks (ICWN'11), Las Vegas, NV, USA, pp. 18-21, 2011 (cit. on p. 89).

[111] K. Zhang, S. Climent, N. Meratnia and P. J. M. Havinga, 'Practical problems of experimenting with an underwater wireless sensor node platform', in Intelligent Sensors, Sensor Networks and Information Processing (ISSNIP), 2011 Seventh International Conference on, IEEE, 2011, pp. 365-370 (cit. on p. 90).

[112] S. Climent, N. Meratnia and J. V. Capella, 'Impact analysis of different scheduling and retransmission techniques on an underwater routing protocol', in Proceedings of the Sixth ACM International Workshop on Underwater Networks, ACM, 2011, p. 8 (cit. on p. 90).

[113] S. Climent, A. Sanchez, J. V. Capella and J. J. Serrano, 'Study of MAC Protocols for a Real Underwater Sensor Network Application', in The 2012 International Conference on Wireless Networks, 2012 (cit. on p. 91).

[114] S. Climent, A. Sanchez, J. V. Capella and J. J. Serrano, 'Simulating MAC protocols under real underwater sensor networks assumptions', in Proceedings of the Seventh ACM International Conference on Underwater Networks and Systems, ACM, New York, New York, USA: ACM Press, Nov. 2012, p. 34, ISBN: 9781450317733 (cit. on pp. 91, 92). 
